\title{
A DIALECTICAL EXPERIENCE IN THE PURSUIT TO UNDERSTANDING: PERSPECTIVES FROM RESOURCE TEAM NURSES
}

by

Niyat Mehari RN, BScN

Ryerson University, Canada, 2013

A thesis

presented to Ryerson University

in partial fulfillment of the

requirements for the degree of

Master of Nursing

in the program of

Nursing

Toronto, Ontario, Canada, 2019

(C) Niyat Mehari, 2019 


\section{Author's Declaration}

I hereby declare that I am the sole author of this thesis. This is a true copy of the thesis, including any required final revisions, as accepted by my examiners.

I authorize Ryerson University to lend this thesis to other institutions or individuals for the purpose of scholarly research.

I further authorize Ryerson University to reproduce this thesis by photocopying or by other means, in total or in part, at the request of other institutions or individuals for the purpose of scholarly research.

I understand that my thesis may be made electronically available to the public. 


\title{
A DIALECTICAL EXPERIENCE IN THE PURSUIT TO UNDERSTANDING: PERSPECTIVES FROM RESOURCE TEAM NURSES
}

\author{
Abstract \\ By \\ Niyat Mehari \\ Master of Nursing \\ Ryerson University, Toronto, 2019
}

Linguistic diversity is increasing in Canada, however, there is a dearth of information related to resource team nurses' experiences with bridging language disconnect in the provision of care. This study aimed to understand the meaning of resource team nurses' lived experiences of language barriers in an urban city in Canada. An interpretive phenomenological approach informed by van Manen, guided this study. Eight resource team nurses were recruited using purposive and snowball sampling strategies. In-depth face-to-face interviews served to unearth the lived experience of being a resource team nurse and the meaning ascribed to language in their everyday practice. The themes that emerged were (a) trying to span the distance, (b) everything comes down to time, (c) desiring shared meaning, and (d) experiencing disequilibrium. The overarching theme was: a dialectical experience in the pursuit to understanding. This study has implications for policy, nursing practice and further research. 


\section{Acknowledgements}

I would like to express gratitude to my supervisor Dr. Sepali Guruge for your continuous support, patience, and guidance. This journey was enriched by your expertise and experiential knowledge in research and content. To committee members, Dr. Susan Bishop and Dr. Oona St. Amant, I am thankfully indebted for your time, guidance, and expertise. Dr. Susan Bishop, your expertise in methodology, phenomenological analysis and meticulous edits were insightful and instrumental throughout this journey. Dr. Oona St. Amant, your insights and feedback have been valuable in elevating my own frequency of critical thought and analysis. The collaborative approach to the composition of this thesis was informative and extremely insightful.

I would like to thank Ryerson Graduate Studies and Master of Nursing program for the support of this thesis through the Ryerson Fellowship Award and Departmental Graduate Award. An extended thank-you to the faculty at the Daphne Cockwell School of Nursing for the support throughout this thesis. Thank-you to Dr. Margareth Zanchetta, thank-you for introducing me to research and your continual support. To Krisel A., Sarah M., and Angel W., I am filled with gratitude to have met such incredible human beings. We inspired, supported and celebrated each other's journeys and I am so very grateful.

Thank-you to the participants who shared their insights and experiences. Your insights have contributed to a deepened understanding of language barriers within the healthcare context.

An endless gratitude to my family. The quintessential heroine of my life's journey, and whose phenomenological narrative is an inspirational account of love, strength, resilience, and dedication, my adey (mother), Lucia Semere, thank-you. To my aboy (father), Mehari Ghermazion, I thank-you for your continuous love and hard work. To my siblings (Mussie, Hanibal, and Sirak), thank-you for your unceasing love, support, and encouragement.

Philippians 4:13 


\section{Dedication}

To persons who are ultimately impacted and further marginalized by barriers reflecting nonlinguistic and cultural inclusions.

To nurses who provide care to ensure the voices of their patients are elevated; you are all greatly appreciated.

To all readers, that you experience your own interpretation in achieving your own understanding of the depth and breadth of this study.

Nothing is more meaningful than the quest for meaning, the mystery of meaning, how meaning originates and occurs --- as well as the meaning of our responsibility for others and the organic, material, and technological world we inhabit.

van Manen, 2014, p. 13. 


\section{Table of Contents}

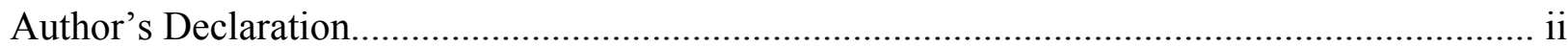

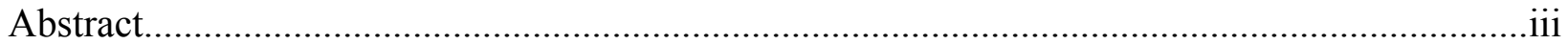

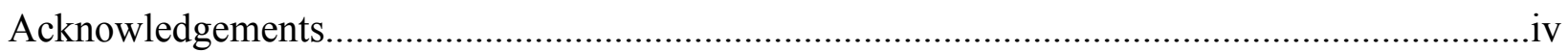

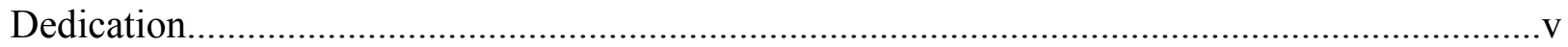

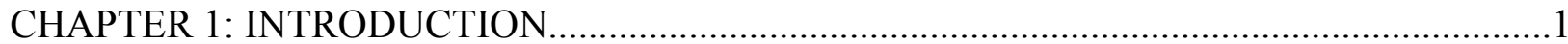

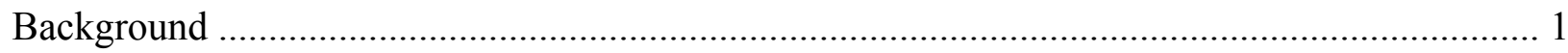

Healthcare Access and Language …………………........................................................ 2

Nursing Resource Teams (NRTs)_.............................................................................. 7

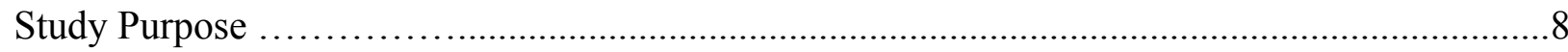

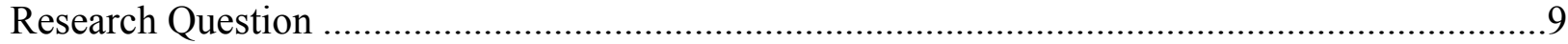

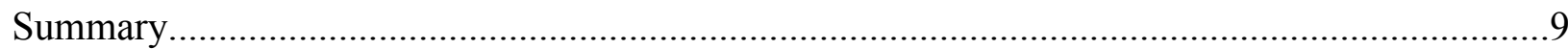

CHAPTER 2: LITERATURE REVIEW ............................................................................

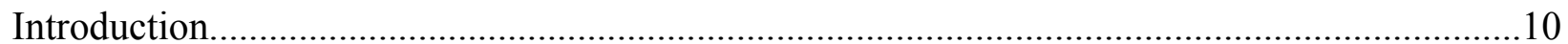

Search Strategy

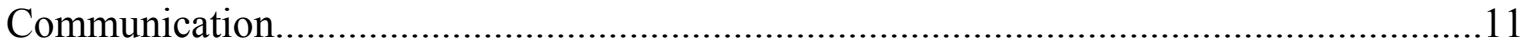

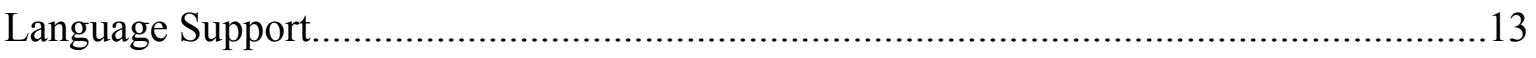

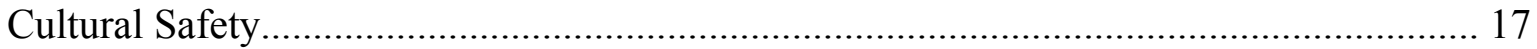

Healthcare Providers' Perspectives..................................................................................19

Gaps in the Literature and Summary ......................................................................22

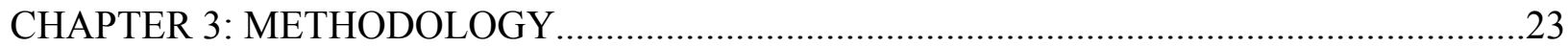

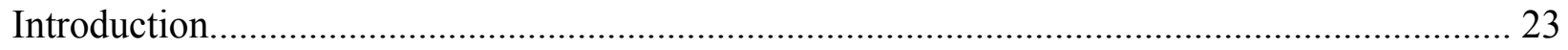

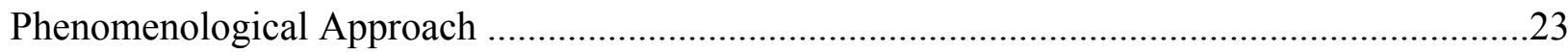




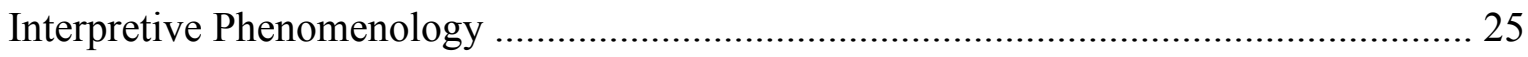

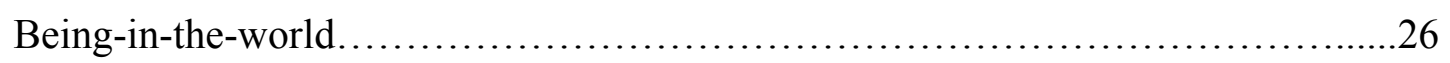

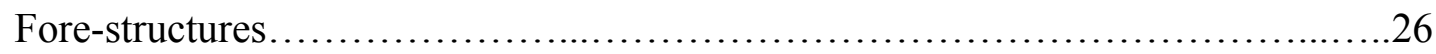

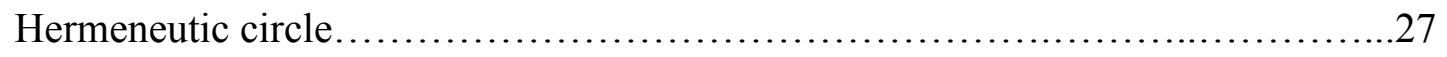

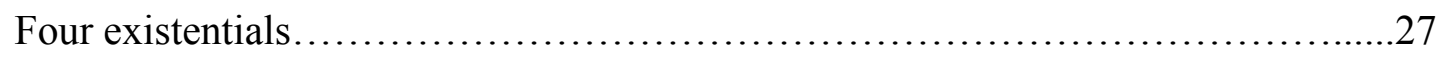

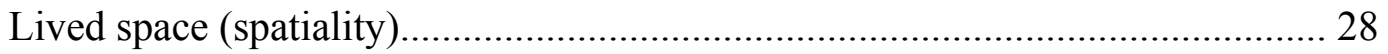

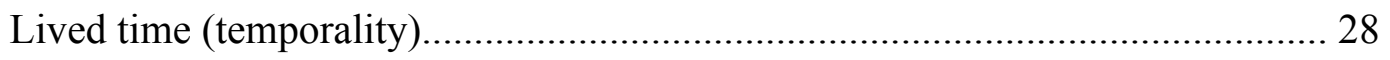

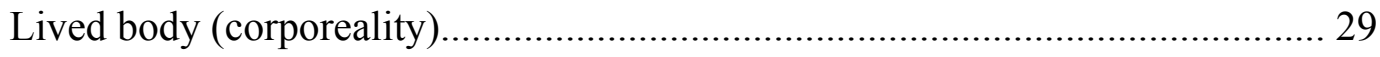

Lived human relations (relationality or communality) ........................................29

Sampling

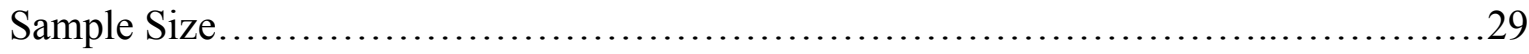

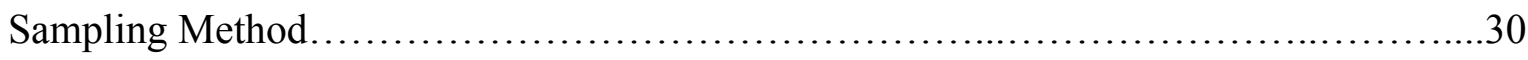

Obtaining Access to the Institution and Participants...................................30

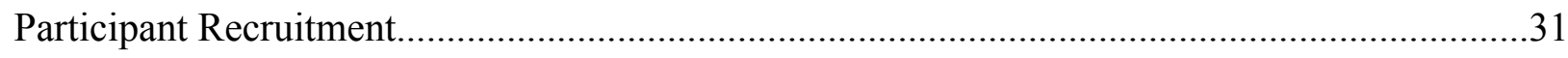

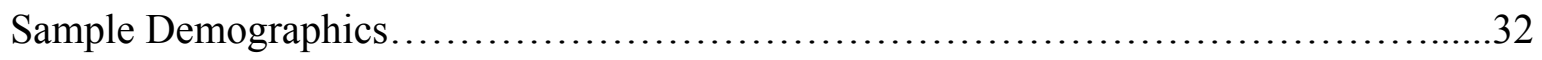

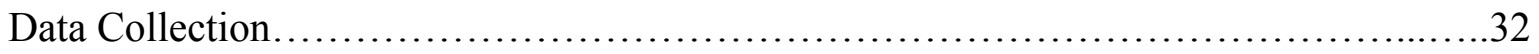

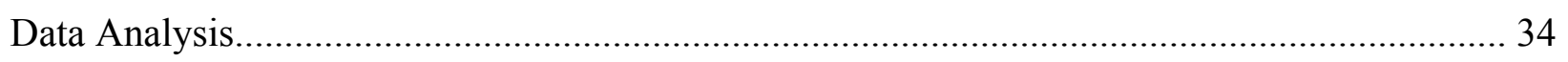

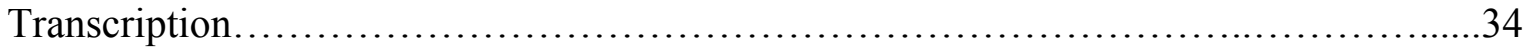

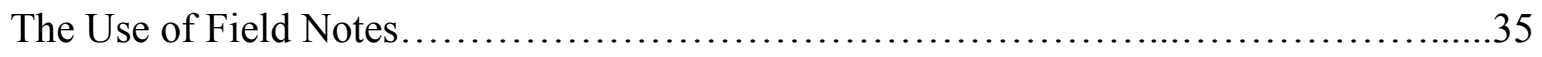

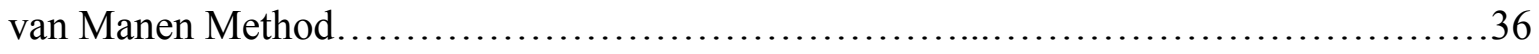

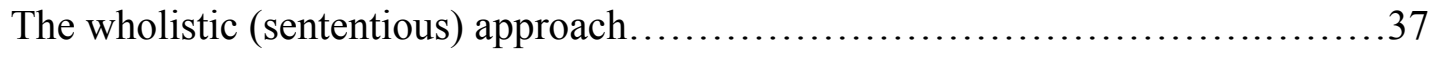

The selective (highlighting) approach....................................... 37

The detailed (line-by-line) approach............................................ 37 
Uncovering the themes and subthemes................................... 37

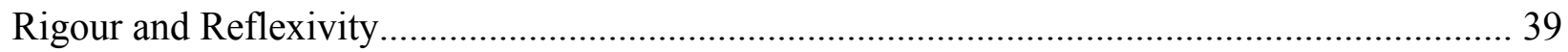

de Witt and Pleog Framework...............................................40

Balanced integration................................................40

Openness...........................................................41

Concreteness..................................................................

Resonance..........................................................42

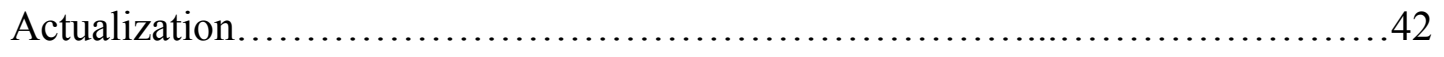

Reflexivity ............................................................43

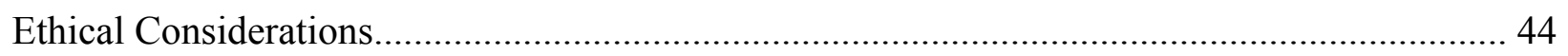

Consent........................................................................44

Confidentiality................................................................ 44

Potential Risks and Benefits..............................................45

Voluntary Participation and Withdrawal........................................46

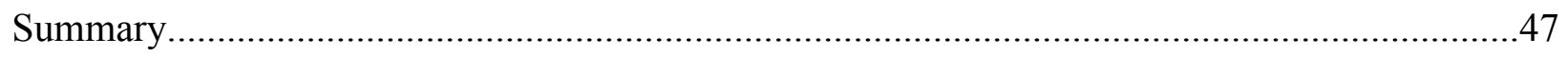

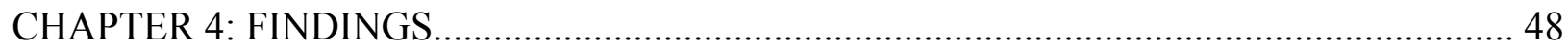

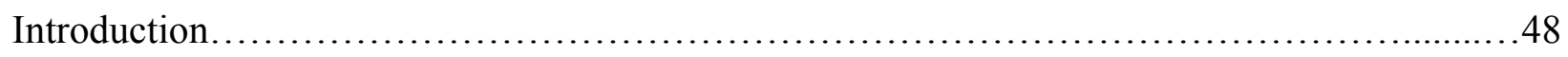

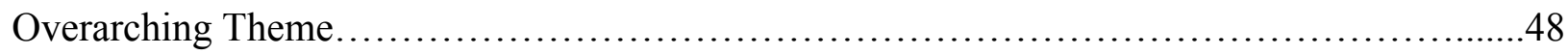

A dialectical experience in the pursuit to understanding....................................... 48

Themes.............................................................................. 51

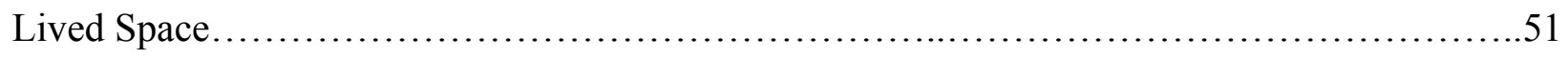

Trying to Span the Distance................................................................................... 51

Playing a guessing game.............................................5 52 


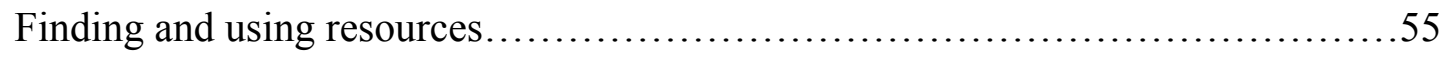

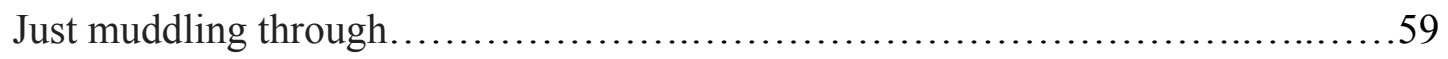

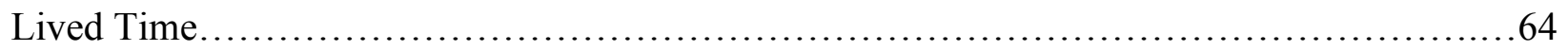

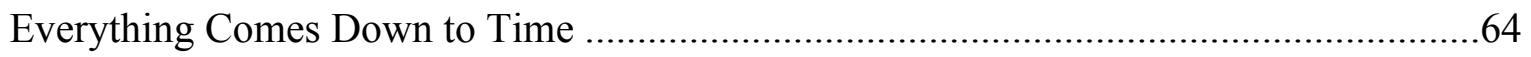

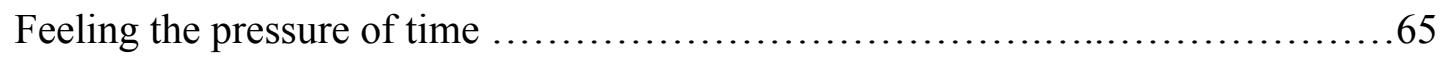

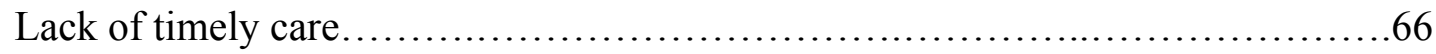

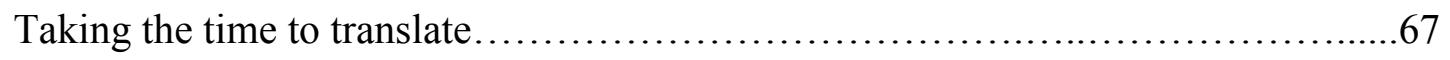

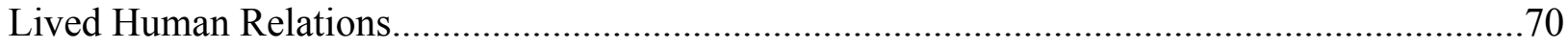

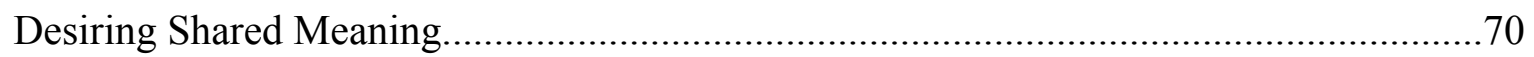

Building emotional connections............................................. 71

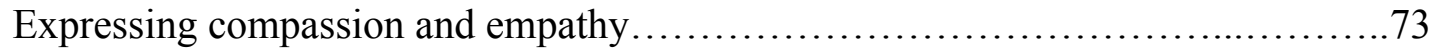

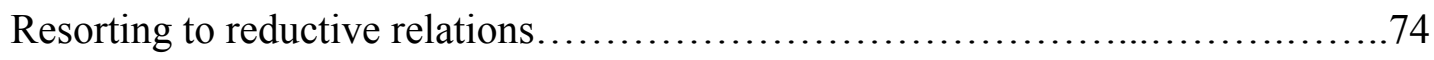

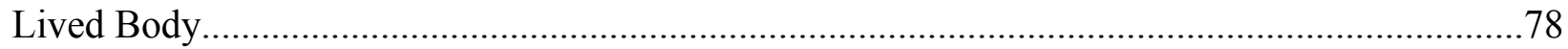

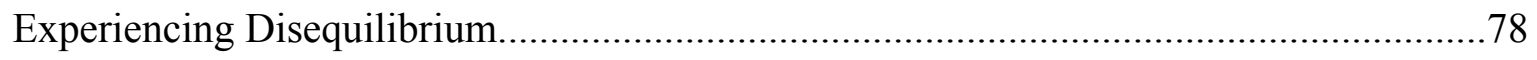

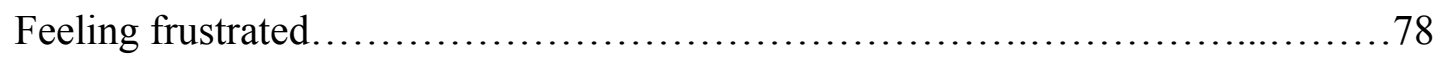

Embodying skills that facilitate care encounters .............................. 80

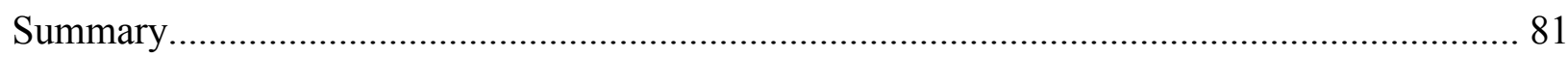

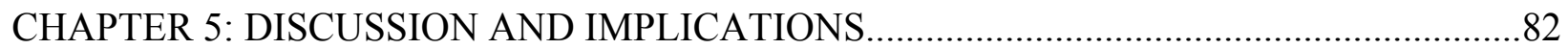

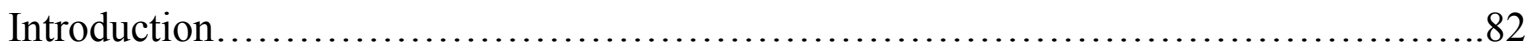

Overarching Theme: A dialectical experience in the pursuit to understanding...................82

Lived Space: Trying to Span the Distance ........................................................................ 83

Lived Time: Everything Comes Down to Time ……………………………………........8

Lived Human Relations: Desiring Shared Meaning.........................................................90 
Lived Body: Experiencing Disequilibrium..................................................................92

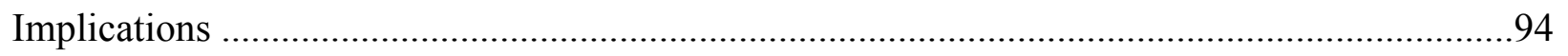

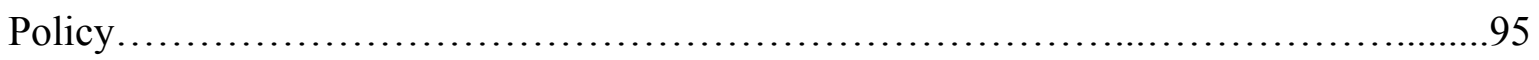

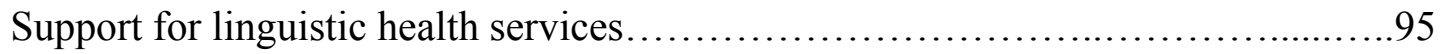

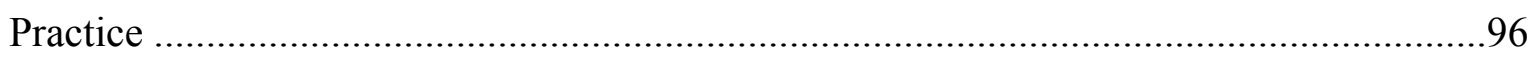

Developing inclusive practice environments ...................................96

Continued professional development............................................ 97

Incorporating equitable practices into workload measures.. ......................97

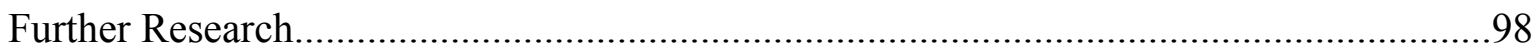

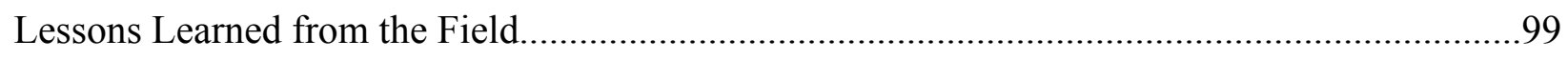

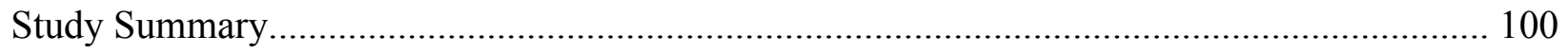

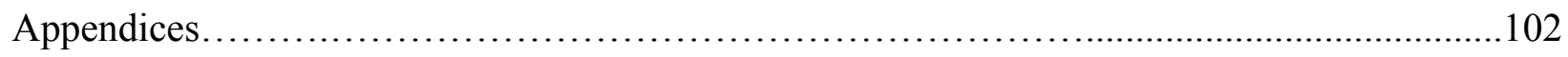

Appendix A: Letter Requesting Approval to Recruit \& Approval Email.............................102-103

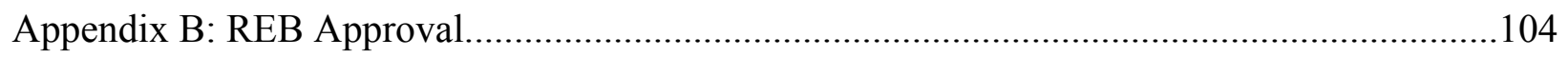

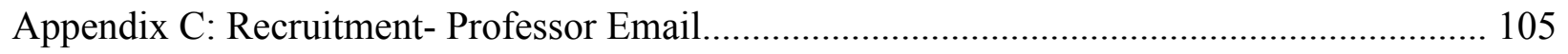

Appendix D: REB Amendment Approval................................................................................

Appendix E: Recruitment- Email/Handout/Speech for Potential Participants.....................107

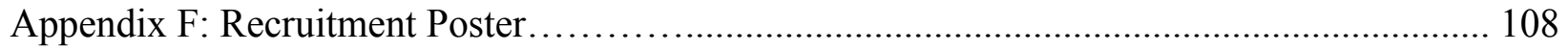

Appendix G: Consent Form ….........................................................

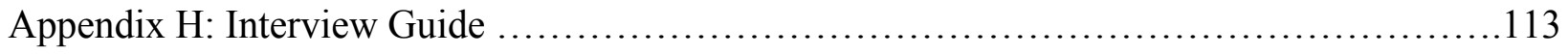

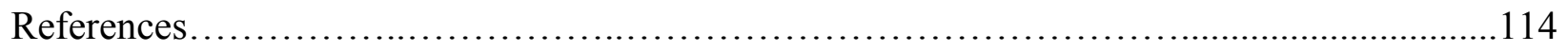




\section{CHAPTER 1: Introduction}

\section{Background}

The diversity of the Canadian population is reflected in the many languages spoken in this country. In total, 215 languages were listed in the 2016 Census (Statistics Canada, 2017a). Approximately, $19 \%$ of Canadians speak more than one language at home, a rise from $17.5 \%$ in 2011 (Statistics Canada, 2017a). In total, up to 7.6 million Canadians stated the language spoken at home does not include English or French, an increase of close to one million people from the 2011 Census data (Statistics Canada, 2017b). Moreover, up to 600,000 individuals in Canada have no familiarity with either English or French when communicating (Higginbottom et al., 2015). Despite this increase in linguistic diversity, system structures continue to be Anglocentric, and bilingual (English/French) at best.

According to Statistics Canada (2017a), languages aside from English or French are considered as, "other languages" (p. 1) and refer to Aboriginal, immigrant and sign languages. Statistics Canada (2017a) provides information on up to 70 Aboriginal languages in Canada. Statistics Canada (2017a) also noted that more than 7.7 million individuals speak a language (aside from Aboriginal languages, sign languages, English and French) native to their country of origin, exclusively or in conjunction with English and French. Although the use of a language other than English or French has increased in every region in Canada, large metropolitan areas have seen the largest increases (Statistics Canada, 2017b).

Immigration is the main driving factor for increased growth in the Canadian population and has influenced linguistic diversity (Statistics Canada, 2017b). In 2016, over 1 in 5 Canadians were immigrants $(21.9 \%$ ), a ratio that is close to the $22.3 \%$ noted during the 1921 Census, a highest level since Confederation (Statistics Canada, 2017c). Immigration has been continually 
rising in Canada (Statistics Canada, 2017c). With growth in immigration in Canada, it is critical to consider the cultural safety in healthcare (Higginbottom et al., 2015), including how services are provided to those who do not speak English or French. Even though Statistics Canada reports growth in languages spoken (Statistics Canada, 2017a), there is however, stagnation in service delivery outside the mainstream linguistic culture (Laher, Sultana, Aery, \& Kumar, 2018; Sultana, Aery, Kumar, \& Laher, 2018).

\section{Healthcare Access and Language}

Healthcare access continues to be of concern in Canada even with a universal, publicly funded approach to healthcare with The Canada Health Act (House of Commons, 1984 as cited in Law et al., 2005). The Canada Health Act of 1984 is the national statute central to the allocation of health service delivery, with the primary goal of guaranteeing the delivery of medically necessary health services to Canadians regardless of the capacity to pay (Asanin \& Wilson, 2008). The principles of (a) universality, (b) accessibility, (c) comprehensiveness, (d) portability, and (e) public administration are the main principles of the Canada Health Act (Asanin \& Wilson, 2008). Although the goal of the Canada Health Act has lessened financial barriers to access, debates persist with respect to the role of other factors, which may hinder utilization and access (Law et al., 2005). Other factors include the social determinants of health such as living conditions people experience, access to and utilization of healthcare services, education, income, socioeconomic status, and social exclusion ${ }^{1}$ (secondary to denial of health services and language services) (Mikkonen \& Raphael, 2010). A language barrier hinders

\footnotetext{
${ }^{1}$ Social exclusion refers to specific populations not being given the opportunity to participate in society and being denied access social, cultural, and economic resources (Mikkonen \& Raphael, 2010). There are four aspects to social exclusion. One of the common aspects is "denial of social goods such as healthcare, education, housing, income, security, and language services" (Mikkonen \& Raphael, 2010, p. 32). The others include denial of participation in civil affairs and exclusion from social production as well as economic exclusion (Mikkonen \& Raphael, 2010).
} 
people's access to healthcare services (McKeary \& Newbold, 2010). Language barriers are a multifaceted social problem that greatly affects people's lives and well-being (Segalowitz \& Kehayia, 2011). Therefore, a consideration of language barrier is significant as such a barrier conflicts with the overarching vision in ensuring optimal well-being for populations in Canada.

Language is the primary method in which patients are able to voice their lived experiences and healthcare providers (HCPs) are able to express their understanding of the lived experience (Brisset et al., 2014; Leanza, Miklavcic, Boivin, \& Rosenberg, 2014; Segalowitz \& Kehayia, 2011). Language is also an important means for ensuring relational nursing care. The basis of nursing practice is communication and conversation (Probst \& Imhof, 2016). The discord between nurse and patient spoken languages is conceptualized as either a language barrier or language discordance in the literature. Language discordance is also conceptualized as what leads to a language barrier (Segalowitz \& Kehayia, 2011). Language discordance is defined as the barrier to effective communication between the patient and HCP due to a lack of proficiency with the same language(s) (Sears, Khan, Ardern, \& Tamim, 2013; Segalowitz \& Kehayia, 2011). Such definitions can disguise the systemic bias of service user(s) to conform to the official languages. The onus is often placed on the healthcare consumer(s) who communicates in a language other than English or French thus marginalizing the service user(s). Thus, the manner in which language is conceptualized within healthcare spaces affects the experiences of patients and also the HCPs. Language barrier in the context of this study refers to the relational distance that exists in language between the nurse and the patient. This term, language barrier, was selected by me and my thesis committee members for clarity and understanding and is used colloquially in practice. It is not selected to reinforce division or exclusion but rather to illuminate existing experiences - including challenges that arise in the 
provision of care.

As cited in the literature, a language barrier serves as a deterrent for individuals accessing healthcare services (Bischoff et al., 2003; Higginbottom et al., 2015; Kalich, Heinemann, \& Ghahari, 2016; McKeary \& Newbold, 2010). Thus, this deterrence to the access of healthcare services highlights a system of inequity (Higginbottom et al., 2015; McKeary \& Newbold, 2010) in which some are socially excluded from healthcare services because of language. Navigating the healthcare system is challenging in itself. Thus, navigating a system in a language other than one's own language can intensify this complexity (Higginbottom et al., 2015) and can result in marginalization. Communication between HCPs and patients is critically important; being admitted to hospital and to triage, having continuous medical assessments performed (Segalowitz \& Kehayia, 2011), being a part of diagnostic and treatment planning (Jacobs, Diamond, \& Stevak, 2010), and being discharged appropriately (Segalowitz \& Kehayia, 2011) depend on this interaction. Therefore, it is important to have appropriate supports and resources available to facilitate a common language in the healthcare setting.

Language barriers are considered one of the most significant factors leading to health and wellness disparities (Brisset et al., 2014; McKeary \& Newbold, 2010). People with limited English ability (a) experience a reduction in access to health services (Karliner, Jacobs, Chen, \& Mutha, 2007) and utilization (Lofters, Moineddin, Hwang, \& Glazier, 2011) of health services, (b) are less likely to receive appropriate care (Jacobs, Chen, Karliner, Agger-Gupta, \& Mutha, 2006), (c) have lower self-rated health (Pottie, Ng, Spitzer, Mohammed, \& Glazier, 2008), (d) and are more likely to dropout (Morrison, Wieland, Cha, Rahman, \& Chaudhry, 2012). In addition, individuals also experience (a) risks of reduction in clinical investigation or increased frequency of unnecessary tests (Ramirez, Engel, \& Tang, 2008), (b) delays in diagnoses (IOM, 
2002 as cited in Coleman \& Angosta, 2017; The Joint Commission, 2007 as cited in Coleman \& Angosta, 2017), (c) longer hospital stays (John-Baptiste et al., 2004; Segalowitz \& Kehayia, 2011) or early discharges (Segalowitz \& Kehayia, 2011), (d) repeated emergency department visits (IOM, 2002 as cited in Coleman \& Angosta, 2017; The Joint Commission, 2007 as cited in Coleman \& Angosta, 2017), (e) greater likelihood of medical errors (Flores et al., 2003 as cited in Jacobs et al., 2006; Segalowitz \& Kehayia, 2011), and (f) poor health outcomes (Pottie et al., 2008).

Individuals who are unable to communicate in the official languages experience inequities in healthcare spaces (Laher et al., 2018). Health equity, as defined by Health Quality Ontario and the World Health Organization, is the ability of all persons to acquire their full potential in health (Health Quality Ontario, n.d.; World Health Organization, n.d.) regardless of residency, socioeconomic status, religion, language, gender, and culture (Health Quality Ontario, n.d.). All people should be able to receive quality care that is fair and appropriate for them (Health Quality Ontario, n.d.). However, linguistic barriers have been found to contribute to the significant inequities in access and use of healthcare services (Bischoff et al., 2003).

Service users with limited proficiency in the official languages of Canada, English and French, have voiced challenges in finding resources and supports in their preferred language of preference as well as quality interpretive services (Chen, Li, Fung, \& Wong, 2015; Lor, Xiong, Schwei, Bowers, \& Jacobs, 2016). Service users also expressed (a) feelings of emotional distress and dissatisfaction (Lor et al., 2016), (b) fear and hopelessness (Poureslami, Rootman, DoyleWaters, Nimmon, \& FitzGerald, 2011), and (c) reluctance to access and utilize health services (Chen, et al., 2015). Service users also recognize that limited English negatively affects their (a) health, (b) ability to navigate the healthcare system, (c) access to and understanding of health 
information (Nimmon, 2007), and (d) opportunity to receive appropriate healthcare (Probst \& Imhof, 2016).

In the hospital setting, nurses serve to provide direct service and contact (Coleman \& Angosta, 2017) and rely on language for good practice. The dearth of information about nurses' experience and insight into providing care for patients whose first language is not English or French is meaningful in addressing the social inequity created by language barrier(s).

Furthermore, nurses have the knowhow to highlight systemic and organizational factors that contribute to inequity as well as identify linguistic resources such as interpreters (Higginbottom et al., 2015; Kale \& Syed, 2010). The concept of social justice underpins the profession of nursing (Matwick \& Woodgate, 2017). Socio-political environments produce or mitigate the climate necessary for equitable administration and delivery of healthcare (Kirkham \& Browne, 2006), and thus nurses have agency for social action and change to inequitable care (Matwick \& Woodgate, 2017).

Nurses' insights are critical for shedding light on their lived experiences with patients where a language barrier is present. The nurse-patient relationship is theorized as a "professional, therapeutic relationship established to meet the needs and health outcomes of the patient, to ensure no harm comes to them and they are supported towards recovery, or a peaceful death" (Feo, Rasmussen, Wiechula, Conroy, \& Kitson, 2017, p. 55). The nurse-patient relationship is a significant relationship in healthcare as nurses spend the most time with patients (Coleman \& Angosta, 2017; Probst \& Imhof, 2016) and the closeness required in the relationship to attend to patients' basic care needs, differentiates it from other types of therapeutic relationships (Kitson, 1993). Even patients consider the nurse-patient relationship to be essential in fostering wellbeing, empowerment and health (Halldorsdottir, 2008). Through numerous interactions and 
actions, nurses aid in humanizing the patients' illness experience and care (Hawley \& Jensen, 2007) through co-participating in the nursing process (Probst \& Imhof, 2016). These interactions require interpersonal skills to provide nursing interventions (Bridges et al., 2013; Feo et al., 2017), which explicitly impact the delivery of holistic care. When nurses provide care that mirrors their personal aspirations and is believed to be best for the patient, they may experience feelings of fulfillment and self-development (Bridges et al., 2013).

Therefore, exploring nurses' insights and experiences with patients is significant to understanding how best to support high quality practice and to focus on enhancing healthcare service delivery (Bridges et al., 2013). Organizational resources such as interpretation services (Higginbottom et al., 2015) need to be available and accessible to nurses as they are limited in their ability to provide optimal care without such supports such as interpretation services. In particular, a group of nurses whose voices are limited in the literature are resource team nurses. Resource team nurses work within various specialties across various units and become aware of unit and organizational initiatives and thus their insights are instrumental to the delivery of quality care (Lebanik \& Britt, 2015).

\section{Nursing Resource Teams (NRTs)}

Nursing resource teams (NRTs) serve to optimize the flow of human resources as a way to provide a cost-effective solution to the nursing shortage (Dziuba-Ellis, 2006; Larson, Sendelbach, Missal, Fliss, \& Gaillard, 2012; Linzer, Tilley, \& Williamson, 2011). Nurses on resource teams are nurses who are hired by the organization to work across different patient areas to fill gaps in nursing staffing (Dziuba-Ellis, 2006), and may be required to work in different service areas during a shift (Hemann \& Davidson, 2012). Because NRTs fill a temporary gap in the system, they are limited in their capacity to establish meaningful 
connections with their patients. Their ability to relate relationally is further compounded when they are unable to speak in a common language with their patients. There is limited literature on resource team nurses' experiences in working with a language barrier between nurses and patients.

Resources team nurses bring forth an array of skills and experiences across a variety of specialty areas that allow for these nurses to be aware of varying unit cultures and organizational processes. These resource team nurses become sources of knowledge and channels of quality nursing practice (Lebanik \& Britt, 2015). By navigating throughout the organization, these nurses interact with various HCPs and have opportunities to fulfil such roles as advocators, change agents, and gatekeepers ${ }^{2}$ (Lebanik \& Britt, 2015). Since resource team nurses are required to work across different specialty areas and at times in more than one unit, they need to be flexible (Hemann \& Davidson, 2012), organized, focused, adaptable, skilled at communication (Lebanik \& Britt, 2015), and have a heightened focus on learning more about the patient, all which serve to differentiate nursing resource team nurses (Lebanik \& Britt, 2015). Lebanik and Britt (2015) also found that nursing resource team nurses expressed the need for immediacy in understanding their patients and unit protocols (Lebanik \& Britt, 2015). Thus, the immediacy to shared language and meaning becomes significant for NRT nurses and thus becomes challenging with systemic inadequacies hindering the manner to which to overcome the language barrier.

\section{Study Purpose}

Resource team nurses are in a unique position as their role on the NRT requires the immediacy of language to the provision of care. However, there is limited literature on what the

\footnotetext{
${ }^{2}$ Resource nurses fulfill the role of gatekeepers, which is linking a patient with the most optimal staff and resources (Lebanik \& Britt, 2015).
} 
meaning of language barrier is to resource team nurses. Thus, little is known about resource team nurses' lived experiences with a language barrier in practice. The purpose of this interpretive phenomenological study was to deepen our understanding of the meaning of resource team nurses' lived experiences of caring for patients when a language barrier is present.

\section{Research Question}

The central question of the study was: What meaning do resource team nurses ascribe to the lived experience of caring for patients when a language barrier exists?

\section{Summary}

This chapter presented the study context including an overview of the Canadian immigration landscape, the link between healthcare inequities and language, and an overview of the role of NRTs. Throughout this chapter, the importance of exploring this topic is expressed. The next chapter provides an appraisal of the current scholarly literature. The themes that emerged from the appraisal included (a) communication, (b) language support, (c) cultural safety, (d) and HCP perspectives. The gaps in the literature review are also discussed. 


\section{CHAPTER 2: Literature Review}

\section{Introduction}

The literature related to nurse and patient language barrier(s) has been explored in different healthcare settings internationally. Empirical work related to the service user experiences has illustrated that there is a lack of inclusivity in healthcare. Throughout the literature, emerging themes included communication as fundamental to effective interactions, language support, cultural safety, and HCPs' perspectives of language barriers. The main concepts pertaining to language barrier highlight the gaps in the literature and how this study will address the identified gaps.

\section{Search Strategy}

A general search was conducted in the Ryerson University Library \& Archives (RULA) to obtain relevant scholarly literature. Keywords specific to the general search included (a) "language discordance healthcare Canada", (b) "language barrier healthcare Canada", and (c) "language barrier and nursing". From this general search, titles were screened and selected based on relevancy, time period (2007-2017), and locale (Canada). The databases searched were (a) ProQuest, (b) Cumulative Index to Nursing and Allied Health Literature (CINAHL), and (c) Scholars Portal Journals. In the databases, key words searched included: "language barrier in healthcare" "language barrier" AND "healthcare Canada" "healthcare access language barrier Canada" and "nursing float pool". The Boolean operator "AND" was used. The delimiters included: peer-reviewed, 2007-2017, and English, Canada, however the time period was extended beyond this range to relevant theoretical and primary articles. A separate search was done of the Journal of Immigrant and Minority Health, as this was the most prevalent journal within searched articles, using the same keywords. 
No literature was found pertaining to nursing resource team nurses' perspectives of language barrier. Therefore, a general search focused on nursing, with different practice settings, was conducted through which 4 articles were located. When the search was expanded to studies focusing on other HCPs with patients, 4 more articles were located. Then, the search was broadened to locales outside of Canada (such as North America, Europe, and Australia), where language barrier in healthcare delivery remain pervasive (Bischoff \& Denhaerynck, 2010; Brisset et al., 2014; Coleman \& Angosta, 2017). Although, literature not based in Canada was incorporated in this review, careful consideration was given to each piece of literature as specifics of healthcare delivery and populations may have differed from that of Canada.

\section{Communication}

A common theme that emerged from the literature is the conceptualization of language as a fundamental element to effective interaction (Higginbottom et al., 2015). The significance of effective communication upholds ethical principles of justice, non-maleficence, beneficence (Meddings \& Haith-Cooper, 2008) and respect for autonomy (Gillon, 1994; Segalowitz \& Kehayia, 2011) in ensuring communication is in sync both ways. Language is a main medium through which a person is able to voice their lived experience and intentions, and to which HCPs are able to express their understanding of the clinical interaction (Segalowitz \& Kehayia, 2011).

Communication among persons is a multifaceted process and is inclusive of verbal and non-verbal relations between individuals (Coleman \& Angosta, 2017; Higginbottom et al., 2015; Northouse \& Northouse, 1998; Segalowitz \& Kehayia, 2011; Wright \& Moore, 2008).

Communication is the point of access for exploration (J. Anderson et al., 2003). In healthcare, communication is often used as a way of informing the patient and delivering emotional support (Árnason, 2000), and also involves what we receive from the patient to better understand them 
and be relational (Brisset et al., 2014; Segalowitz \& Kehayia, 2011). Effective communicative practices can contribute to a positive impact on the emotional well-being (Chan et al., 2010) of both patients and nurses. Interactions between HCPs and patients can be weakened when a language barrier is present (Diamond \& Jacobs, 2010). This can create discrepancies in understanding (Binder, Borné, Johnsdotter, \& Essén, 2012).

Within the healthcare context, a recognition of health literacy levels is also important in the discourse of language barrier (McKeary \& Newbold, 2010). An antecedent to effective assessment of health literacy requires the careful consideration of language (Poureslami et al., 2011). It is therefore important to recognize, assess and address health literacy in order to ensure delivery of quality healthcare to ensure equitable practices are in place when a language barrier is present. Resources need to be put in place in order to foster literacy with regards to treatment and management (Chen et al., 2015).

Barriers to language hinder access to and resources for patients across various healthcare services such as in oncology (Lee \& Vang, 2010), paediatrics (Eneriz-Wiemer, Sanders, Barr, \& Mendoza, 2014), reproductive health screening (Ahmad, Mahmood, Pietkiewicz, McDonald, \& Ginsburg, 2012), respiratory management (Poureslami et al., 2011), as well as in mental health (Brisset et al., 2014; Chan et al., 2010; Chen et al., 2015). Service users with limited English proficiency have noted the impacts of language difficulties on their health when navigating the healthcare system and accessing and understanding health information (McKeary \& Newbold, 2010; Nimmon, 2007; Sears et al., 2013), and thus widening inequities and disparities. Nurses have voiced challenges and frustration in the inability to communicate with patients (J. Anderson et al., 2003; Coleman \& Angosta, 2017). 


\section{Language Support}

Linguistic resources are vital in working within the context of a language barrier. In healthcare, identifying concerns and needs of those who do not speak English is mostly done through informal untrained individuals, known as ad-hoc interpreters, such as family members, HCPs, non-clinical staff, and friends (Brisset et al., 2014; Edwards, Temple, \& Alexander, 2005; Probst \& Imhof, 2016; Ramirez et al., 2008).

The convenience of ad-hoc (untrained) interpreters is discussed in the literature as they can be contacted on short notice; however, issues of neutrality, lack of health literacy knowledge, and accuracy of interpretation arose as concerns (Hadziabdic, Albin, Heikkilä, \& Hjelm, 2014; Kale \& Syed, 2010; Meddings \& Haith-Cooper, 2008; Probst \& Imhof, 2016; Ramirez et al., 2008). The use of ad-hoc interpreters presents as an ethically questionable practice due to possible breaches in confidentiality, issues regarding the accuracy of the information being exchanged, as well as a lack of patient autonomy over the message being communicated on their behalf (Meddings \& Haith-Cooper, 2008). HCPs who interpret have the medical and healthcare knowledge; however, they do not have the training of professional interpreters (Hadziabdic et al., 2014; Probst \& Imhof, 2016) and may not have the linguistic skills to interpret beyond a simple and informal manner (Meddings \& Haith-Cooper, 2008).

Professional interpreters, in-person and/or via telephone, are another option discussed in the literature (Coleman \& Angosta, 2017; Higginbottom et al., 2015; Probst \& Imhof, 2016). The use of professional interpreters is highly valued in reducing communication barriers (Chan et al., 2010; Higginbottom et al., 2015; Whitman \& Davis, 2009), enhancing patient satisfaction, reducing wait times in emergency departments, and generally decreasing healthcare expenditure through appropriate utilization of healthcare resources such as that in diagnostic testing (Chan et 
al., 2010; Hampers \& McNulty, 2002; Ramirez et al., 2008;). However, there are challenges with optimizing the use of professional interpreters, such as in clarifying complex information (Diamond, Schenker, Curry, Bradley, \& Fernandez, 2009; Higginbottom et al., 2015).

The complexities with both ad-hoc and professional interpreters were discussed in the literature. Brisset et al. (2014) further noted that no matter the type of interpreter available there is the potential to alter the dynamics of power, protection, and dominance within the space of the medical meeting. It is therefore important to be cognizant of this potential as it can impact the quality of care (Brisset et al., 2014). HCPs spoke about the difficulty in expressing their empathy using both ad-hoc and professional interpreters which hindered their ability to cultivate the provider-patient relationship, also conceptually termed "therapeutic alliance" (Brisset et al., 2014, p. 1242). Moreover, in Brisset et al.'s (2014) study on the concerns of primary care mental health HCPs who care for allophones (i.e. individuals whose mother tongue is not English or French) in Montreal, interpreters were considered fundamental in overcoming language barriers; however, a great number of HCPs utilized ad-hoc interpreters rather than professional interpreters, as the main barrier to utilization of professional interpreters was attributed to the obligatory administrative process. HCPs considered the interpreter role of the linguistic representative to be the most significant, followed by providing sociocultural information relevant to the patient (Brisset et al., 2014).

It is also important to note that even with the access of professional interpreters, interpreter support services have been noted in the literature to largely remain underutilized in healthcare settings (Higginbottom et al., 2015; Kale \& Syed, 2010). Kale and Syed (2010) argue that interpreter services are not rooted in a quality-assured and standardized method and as such uptake is reliant on the HCP's own understanding and initiative. Nurses have voiced 
dissatisfaction with their own competence level of using interpreters, leading nurses to highly value training in how to best work with interpreters (Kale \& Syed, 2010). Brisset et al.'s (2014) study also uncovered a lack of availability of resources pertaining to language, and a lack of training and transparency of the interpreter role. It is therefore critical for organizations and institutions to include resources (such as concrete and practical information) and outcomes (such as with the process), relating to training for the $\mathrm{HCP}^{3}$ (Brisset et al., 2014). Similarly, in the ethnographic study of immigrant women by Higginbottom et al. (2015), social service and HCPs' perspectives of communication barriers with maternity services in rural Alberta, Canada, there was a lack of utilization of telephone interpreter services. The lack of the telephone interpreter services was attributed to (a) a lack of awareness or acceptance, (b) a lack of a telephone present within the space of care, and (c) an unpredictability to timing of labour and birth (Higginbottom et al., 2015).

Cost is a factor that is often attributed to the lack of appropriate interpreter services in the literature. Interpretive service costs are not covered by the provincial healthcare plans in Canada, leaving these costs to organizations and agencies that may already be overextended in terms of financial resources for interpretation support (McKeary \& Newbold, 2010). Instead, interpretive service costs ought to be addressed at the level of policy and government when establishing healthcare budget plans (McKeary \& Newbold, 2010). Even though the costs initially are higher, the consistent use of interpreters lessens the progression of complications and thus reducing the long-term healthcare costs (Bischoff \& Denhaerynck, 2010; Brisset et al., 2014).

J. Anderson et al. (2003) further states that the use of continuous on-call interpretive services, $\mathrm{HCP}$ clinical skills, and anticipatory planning in practice will greatly help in easing

\footnotetext{
${ }^{3}$ The HCPs in this context include family physician, mental health workers, social workers, psychologists, and nurses (Brisset et al., 2014).
} 
distress of patients who are unable to converse in English, thus optimizing the patient healthcare experience. In addition, patients may not want to disclose highly sensitive information to an additional person, as concerns of trustworthiness and confidentiality may arise (McKeary \& Newbold, 2010; Kale \& Syed, 2010). In Hendson, Reis, and Nicholas' (2015) qualitative grounded theory study on HCPs' experiences in caring for newcomers in the neonatal intensive care unit, HCPs commented that even with interpreters, translating words in situations that were emotionally laden, was highly distressing (Hendson et al., 2015). Furthermore, HCPs noted that interpreters, based at the hospital, were utilized only in urgent situations which created an uncomfortable environment by bringing in an unfamiliar person (Hendson et al., 2015).

In addition, quality of health information and resources continue to hinder non-English speaking individuals in accessing quality information. In Poureslami et al.'s (2011) qualitative study in Vancouver with new immigrants on their experiences with education and effective management of asthma revealed concerns with equitable access to healthcare information. The participants who were from multi-ethnic backgrounds, voiced concerns that the health information hardly satisfied their needs and requested resources in their native language (Poureslami et al., 2011). Participants added that there was a lack of HCPs who spoke their native language (Poureslami et al., 2011).

Ensuring appropriate measures are in place such as professional interpreters for patients who do not speak English is essential in healthcare. In Jacobs, Shepard, Suaya, and Stone's (2004) study on the use and cost of interpreters in four health centers, patients who received care with the new added interpreter services had a substantial increase in their access and utilization of healthcare services and visits to physicians. Coleman and Angosta (2017) note that the removal of language barriers has the potential to (a) prevent medical errors, (b) increase patient 
safety and quality care, (c) reduce visits to the emergency department, and (d) increase patient satisfaction.

\section{Cultural Safety}

The literature pertaining to language barrier (Anderson, Scrimshaw, Fullilove, Fielding, \& Normand, 2003; Chan et al., 2010) and communication often includes discussions of culture (J. Anderson et al., 2003). Culture is foundational to the meaning behind language, styles of interaction, methods of looking for healthcare services, and ways of describing the body and illness (Enarson \& Ait-Khaled, 1999). Enarson and Ait-Khaled (1999) further state that cultures differ with these differing elements. Culture is difficult to isolate from language (Jacobs et al., 2010) and vice versa. Cultural understanding and mutual trust are the prelude to developing a therapeutic relationship (Meddings \& Haith-Cooper, 2008), both of which require effective communication.

In the literature, cultural safety is one of the ways of conceptualizing inclusivity of culture within the HCP and patient interaction. The conceptualization of culture has been criticized for its roots in essentialism (Garneau \& Pepin, 2015; Gray \& Thomas, 2006; Kirmayer, 2012; Williamson \& Harrison, 2010), which is the perspective that culture is unchanging and static (Garneau \& Pepin, 2015) and focuses on cognitive elements of culture such as beliefs, morals and traditions (Williamson \& Harrison, 2010). The essentialist view of culture is the dominant viewpoint in the nursing and health science literature (Gray \& Thomas, 2006; Garneau \& Pepin, 2015). Cultural competence is conceptualized as the HCP attempting to work effectively within the cultural context of the client (person, family, or community) (Campinha-Bacote, 1999; Garneau \& Pepin, 2015), whereas cultural safety allows for the constant critical questioning of the HCP's own cultural identity and how this influences the HCP relationship as determined by 
the person or family (Garneau \& Pepin, 2015; Nursing Council of New Zealand, 2011). The critical questioning of one's own identity is important as the nurse is not remiss from the culture or a-cultural. Cultural safety does not only involve a partnership between two individuals of different cultures but also between the individual and an organization (Garneau \& Pepin, 2015).

Historically and culturally, nurses are connected with others and with society (Garneau \& Pepin, 2015). For nurses to set aside biases in the relationship with patients, entails a conscious effort (Garneau \& Pepin, 2015). Nurses need to consider and widen their nursing lens to include the structures of power that impact the societal representations of health, care and culture (Garneau \& Pepin, 2015). In other words, nurses need to not only reflect on their own biases but the systemic bias that can further marginalize their patients. Nurses need not be responsible for such bias, but at the very least identify biases so they can best advocate for their patients. Employing a cultural safety lens within linguistic practices may highlight the challenges of how to facilitate communication with varying linguistic groups represented in Canada (J. Anderson et al., 2003).

In addition, HCPs should practice with a focus on reinforcing cultural safety and inclusivity to elevate patients' voices and provide equitable care. In Blackford and Street's (2002) study of nurses caring for immigrant women who did not speak English, nurses engaged as co-researchers to explore immigrant women's experiences and analyze their own AngloAustralian culture. The nurses upheld the belief of treating all individuals the same which can lead to the delivery of inequitable care, especially when interpretive services for the immigrant women were required but perceived as time consuming and at times were avoided (Blackford \& Street, 2002). The nurses obtained greater awareness of the need to ensure linguistic support such as professional (women) interpreters were made available for the care encounter (Blackford \& 
Street, 2002). This focus on equitable care for culturally diverse women who were not proficient in English sparked the need to consider inclusivity for diverse cultures (Blackford \& Street, 2002). Similarly, in Hendson et al.'s (2015) qualitative study of HCPs' experiences in caring for newcomers in the intensive care unit, HCPs voiced the need for continued professional development in cultural inclusivity. The need for continued professional development highlights an organizational gap regarding the lack of supports and resources available for staff when a language barrier is present.

For individuals with linguistic barriers to communication, institutions need to recruit staff from similar diverse backgrounds as the population being served, as well as use comprehensive resources and a variety of interpreter services (L.M. Anderson et al., 2003; Chan et al., 2010). These resources and services include having health teaching materials in multiple languages, and creating culturally safe healthcare environments as reflective in approaches, actions and policies (L.M. Anderson et al., 2003). Meddings and Haith-Cooper (2008) stressed that care needs both linguistic support and be culturally appropriate when being provided to a diverse ethno-cultural population. An approach that is culturally inclusive and incorporates bilingual facilitators is appreciated by patients as impactful in their ability to learn and manage illnesses such as asthma (Poureslami et al., 2011). Poureslami et al. (2011) added that healthcare systems need to incorporate the intersections of language and culture in assessing and fostering literacy of health in diverse communities.

\section{Healthcare Providers' Perspectives}

HCPs' perspectives of language barrier in practice provides an avenue in bringing awareness and challenging traditional Anglophone focused discourse (Blackford \& Street, 2002). By exploring HCPs' perspectives on language discourse, systemic and organizational barriers 
were considered (McKeary \& Newbold, 2010; Whitman \& Davis, 2009). In Hendson et al.'s (2015) qualitative grounded theory study, the perspectives of HCPs (i.e. nurses, respiratory therapists, social workers, nurse practitioners, neonatologists, neonatal perinatal fellows, nursing and respiratory therapist students, administrative staff, and dietitians) per from multiple disciplines were explored with a focus on providing culturally appropriate care within the NICU setting. HCPs voiced frustration and perceived the disconnect in communication as a battle and also felt unsatisfied with the care provided because of the communication barrier (Hendson et al., 2015). Similarly, Probst and Imhof (2016) found that HCPs documented barriers in language as a key challenge in the delivery of appropriate and quality healthcare. Renfrew et al.'s (2013) qualitative study on barriers to care from Cambodian patients, direct line staff including physicians, nurses and a dietitian, found that a majority of HCPs also voiced concerns with language as the primary barrier to the management of diabetes. Hendson et al. (2015) added that HCPs voiced disappointment and a sense of failure in not being able to fully converse with newcomer families, thus reducing the quality of care.

In particular, nurses were dependent on communication for assessments and identifying needs to tailor a care plan for the patient (Probst \& Imhof, 2016). The primary step in the nursing process requires a comprehensive assessment of the patient's history and holistic needs which is then used to construct an appropriate nursing diagnosis (Probst \& Imhof, 2016). Ongoing and effective communication with the patient is essential to collaborating on nursing interventions and evaluation, and is fundamental in all phases of the nursing process (Probst \& Imhof, 2016). Ian, Nakamura-Florez, and Lee's (2016) explorative qualitative study focused on the experiences of nurses, the majority of who worked in the emergency department caring for patients who did not speak English. Ian et al. (2016) found that the nurses indicated that their 
interactions with patients who did not speak English were challenging compared with caring for patients who communicated in English. Concerns with the lack of resources for patients who spoke languages not commonly encountered were mentioned by the nurses (Ian et al., 2016). However, the nurses incorporated changes to their practice when caring for patients where a language barrier was encountered (Ian et al., 2016). These changes included (a) increased attentiveness to patient needs, (b) expansion of knowledge of cultures, (c) growth in personal development, as well as (d) improvement in coordinating care and time management (Ian et al., 2016).

Similarly, in Coleman and Angosta's (2017) qualitative study of nurses' experiences with patients who had limited English proficiency, the nurses' desire to communicate with their patients was considered fundamental to the nurse-patient relationship (Coleman \& Angosta, 2017). Nurses expressed a desire to connect and build trusting relationships which manifested into frustration when they were not able to address the needs of their patients (Coleman \& Angosta, 2017). Coleman and Angosta (2017) further identified that nurses encountered challenges when wanting to provide emotional, physical and educational aspects to care. The nurses also wanted to spend more time with their patients as the interaction with a patient where a language barrier is present can require more time (Coleman \& Angosta, 2017).

HCPs have knowledge regarding organizational structure and climate. In Ian et al.'s (2016) study, nurses found that the supportive organizational structure and availability of resources was fundamental in overcoming communicative barriers present in providing care to patients who were non-English speaking. Similarly, in Brisset et al.'s (2014) quantitative study of Montreal based primary care mental health practitioners, only $36 \%$ of practitioners stated that they had access to linguistic resources and only $2.6 \%$ of practitioners reported having received training in 
how to work with interpreters. Even among practitioners caring for allophone patients, there was little difference concerning their awareness of the availability of linguistic resources and little difference in the likelihood that they would cultivate skills in overcoming communicative barriers (Brisset et al., 2014). Organizational resources tailored to linguistic services and training are essential in addressing language related issues in practice (Brisset et al., 2014). Moreover, HCPs working with refugee clients in Hamilton, Ontario stated that there was a lack of funding and that greater attention to linguistic supports needs to be addressed by all three levels of government (municipal, provincial and federal), particularly the federal government (McKeary \& Newbold, 2010). Language barriers in healthcare require the collective participation of multidisciplinary HCPs, patients, politicians, and administrators (Segalowitz \& Kehayia, 2011). Therefore, according to Probst and Imhof (2016), healthcare organizations should provide strategies to overcome language barriers in clinical practice.

\section{Gaps in the Literature and Summary}

The current literature illustrates the importance of language in delivering culturally safe and quality care in certain clinical settings. However, there was no literature on the perspectives of resource team nurses regarding language barriers in the delivery of healthcare. The study addressed the gap of lack of literature focused on resource team nurses' lived experiences with language barriers. In particular, this study offers a deeper understanding of what meaning resource team nurses ascribe to the lived experience of caring for patients where a language barrier is present specific to the Canadian context. 


\section{CHAPTER 3: Methodology \\ Introduction}

This chapter presents the philosophical foundations underpinning this study, methodological approach (chosen method, sampling, participation recruitment, data collection and data analysis), rigour and reflexivity, and ethical considerations. Congruence of the philosophical underpinning of the chosen approach ensured consistency and integrity throughout the research process and with the findings of the study (Mackey, 2005; Reiners, 2012). Interpretive (i.e. hermeneutic) phenomenology was selected as the methodological approach for this study. Interpretive phenomenology aims to describe, understand, and interpret the experiences of participants (Tuohy, Cooney, Dowling, Murphy, Sixmith, 2013; van Manen, 1997) rather than merely describing participants' experiences. Interpretive phenomenology explores the meaning of the phenomenon through interpretive inquiry (Tuohy et al., 2013). My study explored the meaning of language barriers to resource team nurses through the four existentials. The four existentials are foundational to human existence (van Manen, 1997) and thus provided a deeper understanding of meaning of the nurses' experiences.

\section{Phenomenological Approach}

Phenomenology is an inquiry of human experience and of the perception of a phenomenon as it emerges to consciousness (Langdridge, 2007). Therefore, the focus is on "things in their appearing" (Langdridge, 2007, p. 11). In order to know the world, one is to be in the world and the process of research allows for one to become the world (van Manen, 1997). As van Manen (1997) stated:

Phenomenology calls this inseparable connection to the world the principle of “intentionality". In doing research we question the world's very secrets and intimacies 
which are constitutive of the world, and which bring the world as world into being for us and in us. (p. 5)

Phenomenology focuses on human beings and their worlds (Giorgi, 2005). To conduct phenomenological research is to question the manner in which humans experience the world (van Manen, 1997). Phenomenology is thus both a philosophy and a methodology; a framework in studying how human beings experience the world (van Manen, 1997) and a methodology in studying human consciousness (Dowling, 2007; Mackey, 2005; Matua, 2015; Tuohy et al., 2013). Phenomenological research thrives when meanings are recognizable and made clear (van Manen, 1997). It is a methodology that studies how individuals ordinarily experience the world or become mindful of it (van Manen, 2014).

The two main schools of thought in phenomenology are descriptive and interpretive phenomenology (Tuohy et al., 2013). Phenomenology is heavily shaped by the insights of mathematician, Edmund Husserl (Creswell, 2013; Giorgi, 2005) who developed this philosophical approach in the $20^{\text {th }}$ century (Giorgi, 2005). Husserl's philosophical perspectives were grounded in the belief that assumptions are connected to consciousness and are centered by the individual's lived experience (Reiners, 2012). Therefore, to Husserl the curiosity of what is known about a phenomenon is of importance and thus led to the development of descriptive phenomenology (Reiners, 2012). According to Moran (2000), descriptive phenomenology aims to describe the phenomenon. Alternatively, according to Edmund Husserl's student, Martin Heidegger, the exploration of being is of importance, and thus interpretation (i.e. hermeneutics), and not solely description, is essential in uncovering the meaning of the phenomenon (Reiners, 2012). 


\section{Interpretive Phenomenology}

The uncovering of meaning through interpretation is foundational to interpretive phenomenology (Reiners, 2012). The individual is not a separate entity from the world they live in; in fact, their experiences need to be explored with respect to being-in-the-world (Mackey, 2005; Wilson, 2014). In this approach, the researcher is encouraged to contribute to the interpretation and not bracket, a main difference from descriptive phenomenology (Streubert \& Carpenter, 2011). Bracketing refers to the process in which the researcher sets aside any assumptions and/or beliefs in order to present the description of the phenomenon (Tuohy et al., 2013). In interpretive phenomenology, understanding the meaning of the phenomenon is achieved through the sharing of ideas and the co-creation of knowledge between researcher and participants (Drew, 1998 as cited in Reiners, 2012). Therefore, the essence of interpretive phenomenology is the notion that interpretation is essential in our comprehension of experience (Reiners, 2012).

The epistemology ${ }^{4}$ of interpretive phenomenology is about uncovering the meaning of the experience to the person (Flood, 2010) and the ontology 5 is the study of Being or existence (Langdridge, 2007). The person is the expert regarding their own experience (van Manen, 1997), and this reflects the ontological positioning of interpretive phenomenology. Each person has their own unique experience and thus multiple realities exist (van Manen, 1997). Experiences hold different meanings for different people and through interpretive phenomenology discovery unfolds for the possibilities of Being (van Manen, 1997). Each human has their own personal

\footnotetext{
${ }^{4}$ Epistemology refers to the study of knowledge. It explores questions like "What do we know?" and "How we come to know what we know?" (McEwen \& Wills, 2014, p. 12).

${ }^{5}$ Ontology is a branch of metaphysics that pursues the study of being (what exists or what is) (McEwen \& Wills, 2014) and "establishing truth about our very existence" (Langdridge, 2007, p. 24).
} 
outlook which is understood through the lens of personal, experiential and environmental influences (Thomas \& Magilvy, 2011). Furthermore, interpretive phenomenology calls for knowledge to be co-created between researcher, participant and/or research team (Flood, 2010; van Manen, 1997), thus their axiology ${ }^{6}$ (McEwen \& Wills, 2014) is positioned in the study. Thus, all members' values (mine and my thesis committee members) were incorporated in this present study.

This study was underpinned by the main concepts of Heideggerian philosophy including (a) being-in-the-world, (b) fore-structures, and (c) the hermeneutic circle. van Manen's four existentials were also used which are integral to how human beings experience the world (van, Manen, 1997).

Being-in-the-world. Being-in-the-world alludes to the very nature that humans are not separate entities from elements in the world but are actually closely connected (Mackey, 2005). To be mindful of one's specific Being is the foremost way in fulfilling this notion of being-inthe-world (Mackey, 2005) which involves the ability to inquire and wonder about one's existence (Heidegger, 1962 as cited in Mackey, 2005). Inquiring about one's existence and wondering about one's Being allows for Dasein to be acquired, which refers to a state of authentic living (Heidegger, 1962 as cited in Mackey, 2005).

Fore-structures. The fore-structures refer to what is already known prior to the interpretation process and is the anticipation of the exploration of meaning (Mackey, 2005). According to Heidegger, fore-structures were intended to be an inclusive concept, to recognize that interpretation is already happening; however, expression is what is needed (Mackey, 2005). Through this expression, interpretation allows for what is known to be made explicit (Mackey,

${ }^{6}$ Axiology refers to the study of values (McEwen \& Wills, 2014). 
2005). Meaning is derived through this interpretive process and thus allows for the phenomenon to be made known (Heidegger, 1962 as cited in Mackey, 2005). The consideration of forestructure within phenomenological research is different from Husserlian phenomenology whereby bracketing is employed (van Manen, 2014). Husserl conceptualized bracketing in parallel to mathematics "where what is done within the brackets can be kept separate from the operations outside of it" (van Manen, 2014, p. 215). Whereas, Heidegger argued that humans are inseparable with the world, to make such a separation (Langdridge, 2007). It is not possible for the individual to study the phenomenon "in their appearing" (p. 27) and identify meaning in a detached and neutral manner (Langdridge, 2007). Hence, existence needs to be understood in its cultural and historical context and be understood with respect to the role of language which requires interpretation and not simply description (Langdridge, 2007).

Hermeneutic circle. The hermeneutic circle of understanding refers to the interpretive process as one that is cyclical, whereby one's fore-structures are made clear, next considered as a whole of the understanding, and then thought about in different ways (Heidegger, 1962 as cited in Mackey, 2005). The hermeneutic circle is a back and forth flow of the partial and the whole of understanding to achieve deeper meaning (Mackey, 2005).

Four existentials. The philosophical concepts that are significant to the lifeworld $^{7}$ of human beings include van Manen's (1997) four existentials of (a) lived space (spatiality), (b) lived time (temporality), (c) lived body (corporeality), and (d) lived human relation (relationality or communality). These existentials are the "universal themes of life" (p. 302) and are integral to how human beings experience the world (van, Manen, 1997). The existentials can be

\footnotetext{
7 The lifeworld "refers to our everyday existence in and through which we live our lives" (Norlyk, Martinsen \& Dahlberg, 2013, p. 2). The expression of "lifeworld" means that people's realities are shaped by the world in which they live (Flood, 2010, p. 9).
} 
differentiated but not separated, as they form an intricate harmony of the lifeworld (van Manen, 1997). In this study, all four existentials were employed throughout the data analysis process to allow a full interpretation of the nurses' experiences when caring for patients when language barriers are present.

Lived space (spatiality). Space is essential to being-in-the world as all things have a place in this world (Heidegger, 1962 as cited in Mackey, 2005). Lived space roots the individual in a locale (Mackey, 2005) including individual's feelings towards spaces such as home versus a business i.e. work space (Tuohy et al., 2013). The type and features of the space have an effect on individuals (Tuohy et al., 2013; van Manen, 1997). For example, a new setting may render vulnerability, peculiarity, or excitement; whereas the home holds a special spatial experience that is indicative of the very sense of our being (van Manen, 1997). "There are cultural and social conventions associated with space that give the experience of space a certain qualitative dimension” (van Manen, 1997, p. 103). These social and cultural conventions of space are important within the experiences of research participants as the location of the phenomenon being explored provides a greater understanding of the experiences and ensures connectivity to the philosophical underpinning of phenomenology (Mackey, 2005).

Lived time (temporality). Time is an important element to be mindful of in the process of interpretation (Mackey, 2005) as it provides an enriched meaning of the individual's life (Gelven, 1989 as cited in Mackey, 2005). The approach to deciphering and ascribing meaning to situations that happen at a particular time shapes our understanding and awareness (Tuohy et al., 2013). Mackey (2005) adds that time serves as a significant factor in the comprehension of Being and ways of existence. To Heidegger, temporality is essential to what it means to be and our comprehension of the present always comprises our past and forecasts our future (Langdridge, 
2007). Thus, temporality unifies the experiences of the past, present, and future (Mackey, 2005). According to Mackey (2005), temporality ought to be reflected and situated in the research process as it through this orientation that the experiences of time are revealed in the search of ontological understanding.

Lived body (corporeality). Persons have an embodied experience in the world (van Manen, 1997). We meet another person in his or her lifeworld first through his or her body (van Manen, 1997). For example, the naturalness of being may be lessened or enhanced by the simple act of a gaze by someone else (van Manen, 1997). van Manen (1997) further believes that it is through our bodily presence that we uncover and conceal something about ourselves.

Lived human relations (relationality or communality). Lived human relations encompass the interpersonal space we share with others (van Manen, 1997) and asks how self and others are connected in regard to the phenomenon being explored (van Manen, 2014). When people meet, a conversational relation ensues that allows us to rise above ourselves for a sense of meaningfulness (van Manen, 1997). According to van Manen (1997), humans search for relations to obtain a sense of meaning and purpose in life, as such we are always "being- with" (Langdridge, 2007, p. 32).

\section{Sample Size}

\section{Sampling}

In interpretive phenomenological studies the goal is to obtain the in-depth meaning of the experience for a few participants who have lived the experience (Creswell, 2013), to which a wealth of thorough data is uncovered with a smaller number of participants (Patton, 2002). Therefore, the goal is great depth with the attention to context, detail, and nuances (Patton, 2002). The number of participants can range from 5 to 25 (Polkinghorne, 1989 as cited in Creswell, 2013), however sample size is flexible and emergent (Patton, 2002). However, due to 
the limited timeframe to collect the participants in this graduate student-led study, I aimed for a sample size of 5-10 and recruited eight participants.

In addition, data saturation was not an aim in this study since there is no point of saturation with respect to meaning (van Manen, Higgins, \& Riet, 2016). According to van Manen et al. (2016), "in phenomenological inquiry, you open up a question, which becomes bottomless -so it does not make sense to say that you caught all the meaning or meaningfulness of a human phenomenon" (p. 5).

\section{Sampling method}

Criterion sampling, one of the strategies of purposive sampling (Creswell, 2013; Streubert \& Carpenter, 2011) and snowball sampling were utilized in this study. Criterion sampling is the selection of participants who meet a criterion (Creswell, 2013). In phenomenological studies, criterion sampling is commonly used to ensure selected participants have experienced the phenomenon and are capable of expressing their knowledge of the phenomenon being studied (Creswell, 2013). Therefore, criterion sampling was used to find suitable study participants.

In addition, snowball sampling was used to obtain participants through other participants and through other social networks. Snowball sampling involves the identification of participants from people who know potential participants who meet the criterion (Creswell, 2013). In the simplest sense, it is participants obtained through word of mouth.

\section{Obtaining Access to the Institution and Participants}

Support for the study was first sought from the Director of the School of Nursing at the institution (See Appendix A). The director then elicited and received support from the Director of the Graduate Program and Associate Director of the Post-Diploma program at the same institution (See Appendix A). Once support from all three Directors was received, I then 
submitted my research proposal to the Research Ethics Board (REB) at the same institution. After REB approved the study (See Appendix B), I requested support from professors for in class presentations (See Appendix C). Professors were only contacted if they were teaching in the Winter (2018) and/or Spring Term (2018). Shortly after REB submission, an amendment was requested and approved (See Appendix D) to recruit through social networks as a means of increasing recruitment.

\section{Participant Recruitment}

The inclusion criteria for this study were nurses who (a) had experienced a language barrier in their practice as a resource team nurse, (b) were currently practicing on a nursing resource team and currently providing patient care, and (c) were willing to communicate their experience(s) of caring for patients when a language barrier is present.

Recruitment was marketed through an academic institution and through social networks. At the academic institution, recruitment was marketed through three avenues. First, an email (See Appendix E) was sent to the registered nursing students enrolled in the Master of Nursing $(\mathrm{MN})$ and Post-Diploma programs, with a brief description of the study. Second, recruitment was sought through posting the recruitment poster (See Appendix F) on the nursing information board and in the graduate lounge both in the school of nursing. Informational handouts were left in the graduate nursing floor for potential participants to read (See Appendix E). Last, during the Winter and Spring 2018 terms, a 5-minute speech (See Appendix E) was given at the beginning of MN and Post-Diploma classes with support from the individual professors.

An amendment was sought from the REB to include recruitment through social networks (See Appendix D) in order to increase recruitment. Social networks were known to me and were emailed the study description (See Appendix E). 


\section{Sample Demographics}

This study explored the lived experiences of language barriers from eight resource team nurses. Participants ranged in nursing experience from six and a half months to nine years and worked in various hospital units, primarily with the young adult and adult populations. The hospital units included medicine (respirology, oncology, nephrology, cardiology, stroke), surgical (oncology, cardiac, thoracic, orthopaedic, trauma, neurosurgery, otolaryngology, plastics, general surgery, respirology), mental health/psychiatry, psychiatric intensive care, critical care, emergency, rehabilitation, complex continuing care, and palliative care. Participants reported working within a range of 7 to 15 units. At the time of data collection, six participants were working at academic teaching hospitals and two were working at community hospitals. They reported working with patients from multiple linguistic backgrounds. Other demographic details such as age, gender, etc. were not asked to ensure protection of participant identities.

All participants had proficient knowledge in English. Of the eight participants, two had familiarity with French, four had familiarity with a language other than English or French (Urdu and Punjabi, Twi, Tagalog). For two participants English was their second language (Swahili, Mandarin), and for six English was their first language.

\section{Data Collection}

Data collection took approximately three and a half months. Eight in-depth, semi-structured, one-on-one interviews were conducted resulting in a total of approximately seven hours of data. In an interpretive phenomenological study, interviews are used as a means to collect and explore experiential accounts and serve as a source in gaining a deepened understanding of a phenomenon (van Manen, 1997). Semi-structured interviews are the most common data collection method in phenomenological research (Langdridge, 2007). Semi-structured interviews 
provide some structure but allow for participants to openly discuss their views (Creswell, 2013). The interview questions serve as prompts to explore varying aspects of the phenomenon being studied (Langdridge, 2007). One interview per nurse, lasting approximately 45-90 minutes, was conducted with each nurse. Written informed consent was obtained prior to each participant's interview and ongoing verbal consent was maintained during the interviews (See Appendix G). With consent, the interviews were audio-recorded.

The interviews began with role specific and demographic questions which were followed with the opening question, "As you already know, I am conducting this study to learn more about the lived experiences of resource team nurses with language discordance. What has your experience been like with language discordance or language barriers in practice?" (See Appendix H). The questions were open-ended to allow for participants to freely express their perspectives and experiences. Guiding questions and clarifying questions and prompts were used to obtain clarification and further elaboration (See Appendix H). In addition, a list of three to five openended questions (See Appendix H) were prepared and asked if they fit the experiences being shared by the participant or to illicit further understanding. Interviews ended with the question "Is there anything else you want to add?" According to Patton (2002), it is important in interviews to offer participants an opportunity to express their final word. Thus, this question provided the nurses with an opportunity to share any further information about their experiences.

Field notes were used to capture additional information that was not captured via audio recording (See section on The Use of Field Notes). At the end of each interview, I engaged in critical reflective journaling to note any nuances of emotive expressions from the participants' responses, as well as my own personal thoughts and perceptions. These field notes allowed for 
clarity and awareness when reading through the interview data and added depth to the analysis (See section on Rigour and Reflexivity).

\section{Data Analysis}

Heidegger (1962) considered interpretation to be a circular process (as cited in Mackey, 2005). Fore-structures are first made clear, then reflected upon with regards to the whole of understanding of the phenomenon and then fore-structures are reassessed in new ways (Heidegger, 1962 as cited in Mackey, 2005). This process is reflected in the hermeneutic circle where emphasis is on the iterative flow between fractional understandings and the comprehensive whole (Mackey, 2005). Therefore, my data analysis occurred concurrently with data collection.

\section{Transcription}

I transcribed each interview right after the interview which gave me the opportunity of being immersed in the data, which in turn heightened my insights that were emerging from the texts (Patton, 2002). Therefore, transcribing each interview provided me with a deeper understanding of the experiences which were captured in my field notes.

The transcribed interview was analyzed in hard copy format. A qualitative data analysis software program was not used in this study to organize data and in facilitate analysis. Not using a qualitative analysis software program allowed for minimal restriction regarding analysis. According to Langdridge (2007) using a software program may not be helpful as interpretive phenomenology requires greater flexibility in data analysis. The transcriptions were a verbatim report of the interviews and included every "um", "yeah”, silences, pauses, overlaps, expressions, and mimicked experiential accounts, which I noted in square brackets. Personal and organizational identifying information was removed, however, certain words or phrases were 
replaced with general terms (e.g. "interpreter device" used as replacement to the actual organizational name) or phrases and certain words that are common abbreviations within the hospital environment were clarified by including the full word in brackets, such as psych was worded to psych[iatry] to ensure reader comprehension. Furthermore, I chose not to correct participant errors in syntax or pronunciations as Langdridge (2007) stated that it is important to stay as close to the speech of the participants as possible. The transcript in itself is "a move way from immediacy of the interaction" (Langdridge, 2007, p. 74), and thus a correction or "clean up" would further distance the transcript from the actual interview data. According to Langdridge (2007), it may not make sense to further lose information that could potentially have significance to the meaning of the phenomenon for grammatical purity and it could be impolite to "clean up" (p. 74) the language of participants.

\section{The Use of Field Notes}

Field notes were handwritten during interviews, transcription and analysis. Field notes provided another opportunity for me to be immersed in the data during the time between fieldwork and full analysis, which served as an opportunity to get a feel for the data as a collective whole (Patton, 2002) and position this study within a larger temporal and societal context (Phillippi \& Lauderdale, 2017). My field notes helped to keep a record of essential contextual texts like details of the study interviews, their context, as well as interactions which supported the construction of rich, thick descriptions (Phillippi \& Lauderdale, 2017).

Furthermore, field notes provided information that was non-text based which was valuable in understanding the meaning of the experience (Phillippi \& Lauderdale, 2017), thus enhancing my ability to interpret the interview data. For example, in this study, the use of field notes during the interviews allowed for the creation of questions based on what the participant had expressed 
earlier. In addition, I was able to note descriptive visual expressions at certain moments during the interviews that would not have been captured from listening to the audio recording. For example, one of the participants while discussing their experience displayed facial gestures they use when interacting with patients. I drew these facial gestures as they could not be captured via audio recording and the illustration was incorporated into the data analysis.

\section{van Manen Method}

In this study, van Manen's (1997) method of thematic analysis was used to analyze the transcripts. A theme is referred to as an element frequently occurring in the text and attempts are made to uncover these meaningful accounts in the experiences of persons (van Manen, 1997). van Manen (1997) states that themes in phenomenological inquiry are understood as experiential structures that form experience. Thus, an analysis of data is the process of retrieving the themes that arise or that are embodied in the developing meanings of human experience in the text (van Manen, 1997; van Manen, 2014). Thematic analysis is too often understood as an explicit and automatic application of some frequency amount or coding of meaningful terms in texts or transcripts, or other content of protocols or documentary material (van Manen, 2014). "Analyzing" the thematic meaning of lived experience(s) is one that is a multifaceted and creative process of discovery and invention (van Manen, 2014, p. 320).

van Manen's (1997) multi-step approach allowed for the uncovering of experiential themes appearing as commonality. The interpretive nature of van Manen's (1997) approach allowed for the thematic analysis of the NRT nurses' experiences with language barriers. Thus, van Manen's (1997) approach was used as it combines three steps, namely (a) wholistic (sententious), (b) selective (highlighting), and (c) detailed (line-by-line) approach. 
The wholistic (sententious) approach. The wholistic (sententious) reading approach involves attending to the text as a whole (van Manen, 1997) and was used as the first step in uncovering the themes and subthemes in the data of this present study.

The selective (highlighting) approach. The selective approach is also known as the highlighting approach. This step entails reading the associated text several times and asks, "What statement(s) or phrase(s) seem particularly essential or revealing about the phenomenon or experience being described?" (van Manen, 1997, p. 93). As I read the text(s) several times, I highlighted and made comments to text(s) that uncovered meaning of the nurses' experiences with language barriers.

The detailed (line-by-line) approach. The detailed approach, also known as the line-byline approach, entails being attentive to every sentence or cluster of sentences and questioning what each sentence uncovers about the phenomenon (van Manen, 1997). van Manen (1997) asserts that this step allows for the careful consideration of each text in the process of thematic analysis. By being active with the text, the detailed approach ensured that every line was considered and incorporated into the thematic structure of this present study.

Uncovering the themes and subthemes. I first began with reading and re-reading the text(s). Then, I followed with reading the text(s) several times while highlighting and adding comments to what the text(s) or passage was illuminating and made note of the emerging themes. Then, I reflected on each sentence and group of texts about what was being uncovered with respect to NRT nurses and language barriers. I gathered the emerging themes and compiled a list of the corresponding text(s) to the themes. Through a cyclical process of reading and writing, I was able to clarify and engage in reflective practice that allowed for greater meaning to emerge resulting in the discovery of the potential themes and subthemes. 
As I continued to read, I highlighted and further grouped text(s) that were similar in meaning which resulted in a preliminary presentation of nine themes. Once I compiled a list of themes, I engaged in further reflection that was an iterative process into differentiating essential from incidental themes. According to van Manen (1997), while developing the thematic structure, it was important to be aware of and distinguish between essential themes and incidental themes. Through this iterative process, four essential themes and 19 subthemes were uncovered. I met with my thesis committee and engaged in continual discussion and reflection of the proposed themes and subthemes. This collaborative process allowed for a deeper meaning and clarification of the potential themes and subthemes. From our collaborative discussions and continual reflection an overarching theme, four essential themes and 11 subthemes were identified as fitting the NRT nurses' experiences with language barriers.

Therefore, in the process of differentiating between essential and incidental themes, I reflectively considered each theme and asked myself the following questions: "Is this phenomenon still the same if we imaginatively change or delete this theme from the phenomenon? Does the phenomenon without this theme lose its fundamental meaning?" (van Manen, 1997, p. 107). van Manen (1997) goes on to say that the meanings that we come across during the process of analyzing may not be unique to the experience or phenomenon being explored, and thus would be considered incidentally related. The development of the essential themes proceeded with identifying, clarifying and solidifying subthemes that fit with the nurses' experiences and engaged discussions with my thesis committee.

Thematically speaking, it is necessary to search for meaning to what is universal from the particular (Langdridge, 2007). The overarching theme emerged at the very end of data analysis by taking a step back and reflecting and questioning what the overall essence of the findings was. 
van Manen's (1997) data analysis approach assisted me in retrieving the overall meaning of the nurses' stories by moving back and forth between parts of my understanding to the whole of my understanding. Furthermore, the collaborative discussions I engaged in with my thesis supervisor and thesis committee members allowed for deeper insights and understandings of the data and to identifying and distinguishing of essential themes, subthemes and the overarching theme. This collaborative process involved examination, articulation, revision, and reformulation of the themes and subthemes (van Manen, 1997).

\section{Rigour and Reflexivity}

The aim of rigour in qualitative inquiry is to ensure the findings reflect the experiences of study participants (Coleman \& Angosta, 2017). However, the discourse regarding rigour in interpretive phenomenological nursing research has been one with controversy (de Witt \& Ploeg, 2006). Views have been debated in the literature pertaining to appropriate rigour criteria in phenomenological nursing research (de Witt \& Ploeg, 2006; Maggs-Rapport, 2001), disagreements over philosophical interpretation (de Witt \& Ploeg, 2006) and the use of generic rigour criteria in qualitative research (de Witt \& Ploeg, 2006). The use of generic rigour criteria in a qualitative study is challenging because in an interpretive phenomenological study the philosophical underpinnings may not be in keeping with the generic rigour criteria (de Witt \& Ploeg, 2006). A common rigour criteria applied in qualitative studies is Sandelowski’s (1986) criteria of rigour which includes (a) credibility, (b) fittingness, (c) auditability, and (d) confirmability (as cited in de Witt \& Ploeg, 2006). Thomas and Magilvy (2011) state, an appropriate approach to rigour is essential in ensuring high quality research that warrants trust or confidence in the study findings. Thus, de Witt and Ploeg's (2006) framework of rigour criteria conducive to phenomenological interpretive inquiry along with the practice of reflexivity were 
used to ensure rigour in this present study.

\section{de Witt \& Ploeg Framework}

To ensure this study is of high quality and the voices of participants are depicted appropriately, de Witt and Ploeg's (2006) rigour framework was used. The concepts of rigour proposed by de Witt and Ploeg (2006) are (a) balanced integration, (b) openness, (c) concreteness, (d) resonance, and (e) actualization. The expressions of balanced integration and openness reflect the research process while concreteness, resonance, and actualization refer to the research outcome (de Witt \& Ploeg, 2006).

Balanced integration. The concept of balanced integration asks researchers to reflect on three characteristics throughout the research process: (a) the suitability of the discussed philosophical theme with the research topic, (b) ensure a thorough weaving of philosophical ideas and concepts within the study process, and (c) maintain an equilibrium between voices of study participants and the philosophical elucidation (de Witt \& Ploeg, 2006).

This expression of balanced integration was pursued throughout the research process in this present study. I engaged in critical reflection to ensure my study purpose and research question were congruent with an interpretive phenomenological study. Throughout the literature review, results and discussion, the reader will be able to identify the appropriateness of de Witt and Ploeg's (2006) framework to the chosen research approach used for this study. For example, this present study was designed to explore the meaning that NRT nurses ascribe to the lived experience of language barriers. The purpose of the present study was to explore meaning which is reflective of the epistemological positioning of interpretive phenomenology which is set to uncover meaning of experience for the individual (Flood, 2010). The epistemological, ontological and axiological underpinnings have been explicitly discussed in the methodology 
section of this chapter demonstrating congruence between de Witt and Ploeg's (2006) framework and this present study.

Openness. The concept of openness is illustrated through explicit and clear rationales for the decisions made throughout any hermeneutic phenomenological study (de Witt \& Ploeg, 2006).

In this study, openness was conveyed in several ways. The use of reflective journaling was fundamental in allowing me to acknowledge and express my own understandings. The process of decision making throughout the research process is explained throughout this study and served as a trail of how decisions were made. During discussions with my thesis supervisor and thesis committee members, decisions were noted and discussed throughout the research process. As Langdridge (2007) points out, working collaboratively ensures quality assurance and thus maximizes rigour. Therefore, this process of active collaboration with my thesis committee maximized my understanding and further encouraged active reflection regarding both process and content (Langdridge, 2007) and allowed for a deeper understanding of the study data.

Concreteness. The concept of concreteness refers to the manner in which findings of the study are written in a way that positions the reader within the context of the phenomenon making it applicable to their reality (de Witt \& Ploeg, 2006). It is important for the lived-through experience of the phenomenon to be accessible to the reader (van Manen, 2014).

In this study, I achieved concreteness by ensuring the findings were reflective of that of the nurses and their nursing practice. It was important to ensure the experiential details were woven into the presentation of the findings (van Manen, 2014). By explicitly selecting quotes that illustrated the nurses' experiential accounts I aimed to position the reader within the reality of these nurses. Furthermore, contextuality as proposed by Madison (1988) aligns within the 
concept of concreteness as the work of the author is to be considered in its everyday world or its historical and cultural context (as cited in de Witt \& Ploeg, 2006). For example, the notion of time was mentioned by the nurses and in conveying the importance that time had for the nurses, quotes exemplifying lived time were chosen and integrated to support the theme.

Resonance. The concept of resonance encompasses the emotive effect the reader experiences when reading the findings (de Witt \& Ploeg, 2006). "The 'speaking' of language gives us the feeling that we are brought 'in touch' with something and thus 'see' something in a manner that is revealing of its experiential sense" (van Manen, 2014, p. 249). The text(s) establish a "feeling understanding" (van Manen, 2014, p. 249) to which a sense of closeness and proximity is produced with the phenomenon (van Manen, 2014). Within interpretive phenomenology the interpretation process is influenced by one's own epistemological positioning and fore-structures (Mackey, 2005) and thus this would indicate that the meaning of the texts is open to the interpretation of the reader. The presentation of the experiential accounts, from the nurses enriched the reading experience and thus, the felt effect will differ with each reader.

One way resonance was sought, in this study, was to use in-vivo quotes from the participants to illustrate each theme and subtheme. For example, Everything comes down to time was a direct quote from one of the nurses and was also a theme in this study. In addition, in-vivo quotes depicting experiential details were important to include to ensure accessibility of the phenomenon (van Manen, 2014) to readers of this thesis.

Actualization. The concept of actualization refers to the future recognition of the significance of the study findings (de Witt \& Ploeg, 2006) and thus considers the temporal element of interpretation. van Manen (1997) speaks about the continual aspect of interpretation 
as "no single interpretation of human experience will ever exhaust the possibility of yet another complementary or even potentially richer or deeper description" (p. 31). Madison (1988) speaks about the potential for interpretation to be affirmed in the future (as cited in de Witt \& Ploeg, 2006). In the future, readers will continue to interpret the study's data and thus interpretation is not completed at the end of the study (de Witt \& Ploeg, 2006). Thus, the findings in this present study are left to future interpretation by reader(s) of this thesis.

\section{Reflexivity}

Reflexivity is essential in phenomenological research as it allows the researcher to acknowledge their own assumptions, beliefs, and ideas (Berger, 2015; Creswell, 2013; Langdridge, 2007). Reflexivity fosters the understanding of one's own epistemological positioning (Jootun, 2009; Patton, 2002) and brings awareness to the researcher of their personal beliefs, personal experiences, social, political positions (Berger, 2015) and ideological origins of not only their perspectives and voices but that of the participants as well (Patton, 2002). Reflexivity calls for critical self-reflection and "a willingness to consider how who one is affects what one is able to observe, hear, and understand in the field and as an observer and analyst" (Patton, 2002, p. 299). For example, I have been exposed to language barriers in my own professional practice with patients and also while trying to help patients navigate a healthcare system where the language the patient spoke was not "commonly" heard.

According to Berger (2015), it was important for me to acknowledge my own personal stance as engaging in reflective practice enriched the accuracy of my research findings, fostered quality research, and allowed me to pay close attention to the underlying meaning of the texts. In interpretive phenomenology, reflexivity is essential as the process of interpretation of findings is an iterative process between the participants, researcher and research team. Therefore, the 
process of being self-reflective was employed throughout the research process and was demonstrated in my reflective journaling. During and at the completion of each interview and during data analysis, I engaged in active critical reflection to ensure I was aware of my reactions to content, thoughts, emotions and triggers i.e. my own performance (Berger, 2015; Phillippi \& Lauderdale, 2017).

\section{Ethical Considerations}

Ethical considerations noted below included the following: (a) consent, (b) confidentiality, (c) potential risks and benefits, and (d) voluntary participation and withdrawal.

\section{Consent}

Support was granted from the Director of the school of nursing, Graduate Program Director, and the Associate Director of the Post-Diploma Degree Program to recruit through the Master of Nursing and Post-Diploma programs. Approval to recruit participants was then obtained from the REB to ensure participant safety and confidentiality were addressed prior to recruitment. Prior to conducting this study, I completed The Tri-Council Policy Statement: Ethical Conduct for Research Involving Humans course on research ethics (TCPS: 2). Written informed consent was obtained prior to each participant's interview and ongoing verbal consent was maintained during the interviews (See Appendix G). Participants were given enough time to consider their decision and have the opportunity to ask questions or seek further information about the study. Participants were also given the opportunity to choose whether to be in this study or not.

\section{Confidentiality}

Participants were interviewed at a private/secure location on a campus of an academic institution as this provided aural and visual privacy in sharing their experiences. This location 
also had minimal noise and distractions so was appropriate for interviewing and audio recording. The audio recordings from the interviews were erased after reviewing them multiple times and when transcription was completed. I transcribed the interview data using a password protected, non-networked computer to type and store the transcripts. No identifying information was used. Participants and their accompanying transcripts were assigned a unique code i.e. NRT 1, NRT 2, etc., which was used throughout the research process so that no names or identifying information were used in the transcripts or in this thesis. Furthermore, identifiable information will not be used in any publications or presentations arising from this study. The list of participant codes was kept on my password protected, non-networked computer and was also locked in a cabinet in my thesis supervisor's office to which only I had access. At the conclusion of this study, the code list was deleted from my computer and the stored paper copy was destroyed. Transcripts and sealed consent forms will be kept for five years, locked in a cabinet in my thesis supervisor's office to which only I have access, in the event they are required for secondary analysis, and will then be destroyed.

\section{Potential Risks and Benefits}

Questions were asked regarding participants' experience(s) with language barriers as nursing resource team nurses. There was a possibility of discomfort, in that some of the questions may have made participants feel uncomfortable. Nurses were made aware that if any question made them uncomfortable, they could skip that question either briefly or permanently. There was no need to provide a reason for skipping questions, stopping the interview, or withdrawing from the study. Participants were informed that any data gathered prior to a participant's notification of withdrawal would be destroyed immediately upon confirmation of 
their request to withdraw. Not one of the participants skipped questions or withdrew from the study.

As participant recruitment was marketed through the academic institution and through social networks, consideration of issues that could arise in this study was important such as potential for coercion and undue pressure to participate as part of dual role risk (e.g. at the time of recruitment, I was also a Master of Nursing student). To preserve and not abuse the professional relationship, it was important for me to be fully cognizant of the potential conflicts of interest that may have arisen and thus I disclosed the nature of potential conflict prior to the interview. I was a current student at the time I was conducting this study and was also connected to social networks which were also used to recruit participants. Thus, there may have been the potential for participants to have a pre-existing professional relationship with me. However, at the time of recruitment, I was not in a scheduled in-person/online class course or in consistent communication with those marketed through social networks. The consent form provided a full disclosure of all the information necessary for individuals to make an informed decision about whether to participate in this research study or not.

A potential benefit to participants was the opportunity for them to talk about their insights and experiences as resource team nurses. There was no guarantee, however, that nurses would receive any direct benefits from participating in this study. If prospective nurses wished to participate, they received $\$ 20$ cash in consideration of their time, travel, transportation, and child care costs. Participants were not paid to participate in this study.

\section{Voluntary Participation and Withdrawal}

During recruitment, participation was reiterated to be voluntary. Nurses could choose whether to be in this study or not. As stated above, nurses were made aware that if any question 
made them uncomfortable, they could skip that question. Participants were made aware that they may stop participating at any time and no rationale was needed. Due to the nature of limited time to conduct this study, withdrawal from this study was not possible after 2-weeks from the interview being completed, and thus withdrawal, could have occurred any time prior to this 2week period. Any data gathered prior to a notification of withdrawal would have been destroyed immediately upon confirmation of the participant's request to withdraw. However, no participant skipped questions or withdrew from the study. Participant's choice of whether or not to participate would not influence their future professional relationships with the academic institution or with me. The consent form provided a full disclosure of all the information necessary for the prospective participants to make an informed decision about participating in this study.

\section{Summary}

The interpretive phenomenological philosophical foundations and methodological considerations have been discussed in this chapter. The methods (chosen method, sampling, participation recruitment, data collection and data analysis) were also discussed. van Manen's (1997) method of thematic analysis was the chosen method of data analysis and is also reflected in the subsequent chapters (Findings and Discussion). Throughout the research process, rigour was sought using de Witt and Ploeg's (2006) framework. The ethical considerations were discussed in ensuring participant safety when conducting this research study. The following chapter presents the findings of this study. 


\section{CHAPTER 4: Findings \\ Introduction}

This chapter presents the overarching theme and the four essential themes that emerged from the data of this present study. Findings were informed using van Manen (1997) four existentials of (a) lived space, (b) lived time, (c) lived human relations, and (d) lived body. The themes and subthemes are presented within each of these four existentials.

\section{Overarching Theme}

In this study, a dialectical experience in the pursuit to understanding was the overarching theme that exemplified the lifeworld of theses nurses and their experiences with language barriers. A dialectical experience in this study refers to the opposing nature of nurse-patient interactions where a barrier to language was present in the pursuit to understanding patient needs.

\section{A dialectical experience in the pursuit to understanding}

The overarching theme, a dialectical experience in the pursuit to understanding, emerged from the experiences of the resource team nurses as they discussed the varying elements encompassing their experiences with patients when language barriers are present. They desired an emotional connection, shared meaning, and tried to span the distance between themselves and their patients, but at the same time the nurses were presented with difficulties in overcoming the language barrier.

The pursuit to understanding is exemplified in how nurses conceptualized the importance of their engagement with their patients to deliver person centered care and to ensure patient safety. Below are two excerpts from NRT 1 and NRT 3, which discuss the importance of the pursuit to understanding their patients' needs. NRT 1 discusses the importance of understanding 
patients to be able to proceed with interventions and referrals such as spiritual care: "To go and emotionally support people [patients] . . but even referring people [patients] to them [spiritual care] requires you to have a basic understanding that they [patients] are having a hard time" (NRT 1). Thus, language serves as the medium to facilitating understanding in order to address patient needs. Similarly, NRT 3 echoes the purpose of language as understanding, "I find that . . . the purpose of language is understanding, um, communicating to understand each other" and continues by discussing the importance of language within the healthcare context:

It's [Language is] crucial in medicine. It's crucial in a way that, um, we understand what is facing our patient. It's crucial that we need to know, Um, it's crucial that we do not miscommunicate what the patients are telling us and it could lead to a lot of misdiagnoses. (NRT 3)

NRT 3 elaborates further by adding:

It's [Language is] significant because it's a form of communication and, um, that's how we understand our patient. That's how we know why the patients are there. That's how we can serve them better. That's how we can help with whatever they come there with so they come home, um, healthier. . . It's very significant because if there is any misinterpretation, misunderstanding then, um . . . errors can happen just because, just because of the language [barrier].

The above excerpts demonstrate that the pursuit to understanding is significant and necessary to be able to emotionally support patients and ensure patient safety. In addition, because of the nature of their role on the NRT, nurses voiced having difficulty establishing rapport with patients, building emotional connections with patients and experienced the 
uncertainty of what to expect with respect to the unit environment and patient assignment. As NRT 6 explained:

You're at different areas like every day, there's not much of a rapport with the patient you met versus if you're on the same floor. You have probably a better understanding, a better rapport with that patient if you ... don't speak that language. Yeah, so I think that's how I feel about being on the NRT and have the whole language barrier.

It is important to note, however, that when nurses and patients communicate with the same language, understanding is not necessarily achieved. The intricacies of language were expressed by NRT 1: "A lot of us [nurse and patient] don't understand each other [tone softens]." The excerpt from NRT 1 addresses the differences in the nurses' cultural positioning, (i.e. political, social, economic etc.), that the nurses embody. Furthermore, capturing the cultural undertones to the position of their patients' health needs, the nurse-patient interactions were challenging. The nurses discussed the nuances of culture having a significant impact when trying to understand their patients. NRT 3 discusses the element of culture as influential to the meaning of language: "Behind their [language] is a culture" and thus understanding culture, is also important. NRT 3 elaborates on the importance of understanding instead of jumping to conclusions:

“Understanding why people [patients] are doing what they're doing instead of just coming with a conclusion." Thus, the complexity to attain meaning in language is uncovered as important in the nurse-patient relationship.

This overarching theme of a dialectical experience in the pursuit to understanding aligns with the nurses' lifeworld and was exemplified in each of the four existentials of lived space, lived time, lived human relations, and lived body. 


\section{Themes}

Through data analysis, four essential themes and their respective subthemes were identified. The four essential themes were (a) Lived space: Trying to span the distance, (b) Lived time: Everything comes down to time, (c) Lived human relations: Desiring shared meaning, and (d) Lived body: Experiencing disequilibrium.

\section{Lived Space}

Lived space roots the individual in a locale (Mackey, 2005) and considers the feelings individuals experience towards spaces (Tuohy et al., 2013). Therefore, the type and features of the space have an effect on individuals (Tuohy et al., 2013; van Manen, 1997) and is thus more than a three-dimensional space. An important element of the participants' lived space was highlighted with regards to distance, in other words, participants trying to span the distance emerged as the meaning of their experiences in their interactions with patients.

\section{Trying to Span the Distance}

Language was described as important to building bridges and is what serves as a connection between the nurse and patient. Several NRT nurses discussed language as being the bridge that helps to span the relational distance between themselves and their patients. NRT 6 spoke about language as a "tool of communication":

Well language itself is a tool of communication, right, but like I think for nurses like a tool ... for us to provide better care. It's what, um, what connects ... it's what puts a nurse and a patient on the same page essentially. . . it's definitely essential for like nursing type of work and it gives us the rapport with the patient, um, and makes sure we provide safe care to the patient, and allows us to do things from a patient's perspective . . . patient centered care.... Language is [the] most important thing, we have to understand 
the patient before we could establish all of this, right. It's definitely very fundamental, it's very essential for nursing practice.

NRT 7 referred to language as "the bridge between the nurse and the patient" by saying: Language is usually the first thing, that's the first way people communicate ... it's like the bridge between the nurse and the patient. Sometimes the language isn't there, that bridge is either completely cut or it's wobbly or it's not straightforward.

The bridge nurses attempted to create was one where they wanted to establish a shared meaning, however rudimentary methods of communication left nurses resorting to reductive and overly simplistic language. Therefore, to capture the patient voice was challenging and free of nuance or complexity. The nurses in this study viewed language as the means in which to understand the patient and provide care that is person centered. When nurses were not able to directly engage with the patient because of a language barrier and were not connected to necessary resources to facilitate communication, the distance between the nurse and the patient was widened and the fragility of the bridge was amplified. Thus, these nurses were trying to build bridges in trying to span the distance between themselves and their patients.

The degree to which this relational distance was lessened or widened depended on what nurses did and what supports and resources were available for the nurses. The subthemes of trying to span the distance were (a) playing a guessing game, (b) finding and using resources, and (c) just muddling through.

Playing a guessing game. Nurses voiced difficulty in fulfilling their duties as a nurse. Primarily, completing patient assessments was noted to be a challenge. Nurses were aware of the importance of obtaining subjective information from the patient to develop the plan of care. As NRT 6 noted, "It's the two components: objective and subjective" while NRT 8 stated that a 
nurse "can't go based on someone's actions or just how they look, right. There's specific details the patient can convey." Nurses expressed their desire to understand patients' perspectives about their personal health as areas difficult to explore. The nurses expressed that this difficulty was heightened when needing to know the "why" to provide clarification and to further their understanding of the patient's subjective voice. NRT 1 discussed the importance of exploring why and discussed an exemplar of when a patient did not want their medication.

Like someone confused and doesn't want to take their medication, like you'll still try and give them their medication. But someone who's with it and there's a language barrier and they don't want their medications kind of do have to explore why, um, cause there could be very good reasons.

In addition, developing mutual understanding of symptom management with the patient was expressed as a challenge. NRT 5 explains the difficulty in obtaining assessment information in such spaces as psychiatry. In addition, the need to co-create and coordinate the plan of care with the patient was expressed as challenging. For example, the following narrative from NRT 5 highlights the difficulties of assessing and planning care for patients when a language barrier is present.

But it's the assessment part that is difficult. And then especially in psych[iatry] where there is so much talking going on, um, but yeah like asking about pain, any symptoms if someone had a lower GI bleed, if you just want to know if they're having stool especially if they're ambulatory and doing it in the washroom and flushing it down, right. Like it becomes this situation where we're like, “oh, you need call us so we can take a look to see if there is any blood" or anything like that, right, so these practical things. 
To span the distance between themselves and their patients, nurses voiced the need to be engaged in a process of questioning that nurses referred to as the guessing game or "trial and error". This guessing game was a quest to ensure the patients' needs and concerns were captured. Nurses spoke about going through a series of questions in the hopes of identifying the patients' needs and concerns. As one NRT nurse explained, "We're playing a guessing game for a little while and it's like oh, all you wanted was water but without the ice, like you don't like the ice and you just want us to bring water without the ice" (NRT 1). NRT 1 adds that the guessing game is enacted in moments where it is perceived that the interpretive resource is not needed such as during the night and attempting to identify patients' needs, "Despite not needing translation in that exact moment because it's in the middle of the night and they're trying to get up and you are like, 'Why are you trying to get up?' [uses hand movements] 'Bathroom?' 'Boredom?' 'Pain?’”

Another NRT nurse added that she had come up with "different guesses of what they [patients] want .... [I] have to do the legwork of figuring out 'do they need water?' 'Do they need to be positioned this way?' 'Is something bothering them?' 'What's bothering them?'” (NRT 2). NRT 1 further explained that patients often stopped requesting things they needed or wanted by stating:

Some people [patients] will stop asking for some things that they don't require as urgently just because they don't want to deal with the guessing game. Oh like, "You called me in here" [but] we don't communicate very well [laughing]. "Is it water?" "Food?" "Toileting?" "Are you uncomfortable?"

As a result, NRT 1 found that, "sometimes peoples' [patients'] wishes are not necessarily $100 \%$ respected, um, depending on what we're trying to do." Moreover, at times interactions 
would result in the nurses resorting to guesses and according to NRT 1 "making assumptions" regarding what the nurses considered the patient was voicing. NRT 1 further elaborated by stating:

I have a good general idea what people [patients] may want in hospital and when I really can't speak to them I try to just make sure they can reach everything [uses hands], they still have water, things get opened, you know some of our containers for food are hard to open and I'm like "that could be why someone could call me."

Here, the assumption of patient needs is voiced by NRT 1. In addition, NRT 6 discussed the nature of their experience in the area of pain assessment and management in the following narrative:

I kind of have to estimate sometimes because I can't get a full description of how much their pain is and that's where it's kind of like it's not so black and white it's kind of like, “oh, okay, this is based on what it seems like" and . . I don't know sometimes. I'm not sure I'm not giving them enough pain medications . . . but like if they're a cancer patient and in a lot [of pain] and they don't really show it, I'm just basing on what I see and then not giving enough and they might be suffering more than they should.

Finding and using resources. This subtheme emerged as participants voiced their experiences with finding and using resources. This subtheme of finding and using resources addresses the nurses' creative abilities in finding and using what resources they needed including using mobile apps Google Translate, as well as relying on their colleagues and the patients' family members and friends. Nurses relied on patients' families and friends as well as their own collegial relationships as a way of capturing and connecting to the patients' voices. Capturing and connecting to the patients' voices was the nurses' primary way of attaining meaning. 
Nurses voiced their dependence on patients' families and friends in obtaining information that would help them in bridging the gap between themselves and their patients. NRT 7 expressed this dependence by sharing, "Usually family are readily available resources to help overcome that barrier." Similarly, NRT 6 discusses this reliance as well in learning about the patient by adding that, "family and friends, that's definitely the best way to go for me to get a better idea of the patient." Nurses appreciated the interpretive assistance they received from family members as family involvement was considered to be most helpful in providing baseline information. NRT 6 summarizes this feeling by saying, "if you have the chance to speak to the family you get to understand the patient's history" as well as changes in the patient's baseline such as cognition, and connecting health information to personal experiences of the patient. Nurses appreciated the help in identifying and clarifying cultural nuances and patient perspectives to ensure a safe environment was established for the patient. However, family dynamics and the complexities, such acts as withholding information, were discussed by the nurses. Nurses who worked in psychiatry voiced the cautious approach employed within this space with using professional interpreters over family:

But, um, in psychiatry, they really do want someone who is a trained interpreter because family dynamics can make a big difference in terms of the kind of information that you get. Um, and like there are times you don't necessarily want the family to be part of the interview. Might speak to them both separately and then altogether because you can get different versions of the same story and you don't want them to have like big back and forth and you're not understanding but like, "That's not how it happened" "Oh, yes it did. . .. "What's going on?" [laughing]. Um, because you know the family was remembering 
something that the patient is not or that they experienced [it] very differently, um, or yeah there is a stigma. (NRT 1)

As families served as the bridge between the nurse and patient, nurses talked about familial influence regarding patient planning and care, as families bring their own lifeworld and interpretation into the engagement. NRT 7 discussed witnessing an experience of another nurse caring for an elderly patient who did not communicate in English and who was experiencing cognitive decline. The family's decision to have the patient restrained conflicted with the perspectives of the nurses:

She was confused, had dementia and her family usually stays over but I guess for some reason her family this evening hadn't. So, she was sundowning [symptoms of confusion and agitation heightened during late afternoon and evening] and she was just getting really agitated. We had tried bringing her to the front desk . . . tried everything we could to try to piece out some of the words, because she was mixing her English with her mother tongue. So, we were able to pick out pieces of what she was saying but we still weren't able to get completely to her because the communication was broken because of the language barrier. Um, so it got to the point where we called the family again and asked them to come in and they came in ... However, they still went home that night and the patient was up all night. ...We don't like putting restraints on ... so the nurse had asked [the family], "okay, so if you're unable to stay, is there something you're okay with us doing that can help ensure that she's safe or that she gets a full sleep?" ... . The family didn't want that [Ativan] ... but they were okay with restraints as a last resort. . . . Just having that language disconnect, I didn't know . . . if that was the right call. I didn't know what the patient needed at that moment. 
NRT 7 continued by sharing, "I don't know if that was just my culture as a nurse being like 'she [patient] needs her sleep' like 'you [primary nurse] should probably be giving her the Ativan and then foregoing the restraints' because the restraints would probably get her more agitated."

Nurses discussed that their assumptions are that family members represent what the thoughts of the patient are and to which the nurses trust is what is being communicated. However, the nurses experienced confusion at times regarding the families' interpretations compared to what the nurses observed and perceived from their nursing assessments. NRT 8 expressed this complexity and the fragility of the nurse-patient relationship when relying on the patient's family to interpret:

Sometimes it's hard because I feel like ... sometimes it's good the patient's family is there but sometimes I also feel like they're preventing us from providing the right care if they're not giving us the right information or if they don't [want] the patient to receive certain types of medications.

In addition to family and friends of the patient, colleagues were utilized and appreciated by nurses for their interpretation skills. NRT 6 voiced an appreciation for the assistance from nursing colleagues with diverse linguistic backgrounds. This nurse found that by getting "one of my co-workers that speaks the same language, that always helps. Like that's always the best if someone could speak the same language and translate." In addition, one nurse voiced the need for diversity among the nurses who are providing care across units by stating, "The demographics of the nurses ... aren't really proportional to the demographics of the patients" (NRT 1). This reliance on colleagues to provide interpretation was considered important for these nurses. 
Nurses preferred resources to be in close proximity to their care and thus perhaps preferring the minimization of physical distance in accessing resources. One such resource was utilizing mobile apps such as Google Translate. The creativity and ingenuity in using Google Translate was just another way the nurses tried to span the distance in their interactions with their patients. Participants were aware of the potential validity and organizational restriction concerns with Google Translate and at the same time appreciated the convenience of this app. As one nurse said, "Something that I found very useful is like using a phone app like Google Translate" (NRT 4).

Just muddling through. Nurses voiced challenges with finding and using organizational supports and resources in their lived space as evidenced by, "Most people [nurses] will just muddle through" (NRT 1). The importance of interpreter services as a way for patients to convey the specifics of their health journey is expressed by NRT 8 , "There's specific details the patient can convey if you had the right tools to like convey about their health.” Thus, nurses not being connected to supports and resources speaks to the lack of a bridge increasing the distance between nurse and patient. The supports and resources varied depending on the organizational structure. Most nurses who worked in academic teaching hospitals were aware of the portable mobile "hotline" interpretation services whereas nurses working in community hospitals stated they were not aware of resources in certain areas within the hospitals.

Most nurses made mention of the financial cost of interpreter resources across both types of organizational structures. One of the two nurses who worked in a community hospital setting stated that only three interpreter devices were available in the entire hospital, "cause they're expensive. They're not cheap.... So, like if you want it, you have to go to another unit where it's actually been stored, to get it" (NRT 3). Another nurse (NRT 7) explained that interpreter 
services or devices were centralized in the emergency department. This nurse exclaimed, "I know emerg[ency] usually has interpreters or they have call-in lines or telephone lines. You could call an interpreter in or we use a system where you send a mass email saying 'an interpreter needed"'. The use of organizational interpreter devices in the emergency department was discussed by the nurses who had worked there, as it is a department of initial contact of patients to the healthcare system. The nurses who worked in community hospitals mentioned that interpreter services were utilized more in the emergency department. The limited resources in community hospitals were a challenge in creating a language bridge between nurse and patient. NRT 7 (who was trained at a larger hospital) explained:

Maybe the experience of ... language would probably be different if it was more, um, like I don't know, like central or at a teaching hospital where they do have those resources that are available. But we're a community hospital. It's [interpreter services] not [available] ... when there is a language barrier.

The importance of communication is heightened within the lived space of the NRT nurse. As nursing resource team nurses, some nurses noted differences with usage of resources across various services. Whether to use these resources also depended on the nurse's understanding of the patient and how important the intervention. NRT 4 discussed the differences across units at their organization in the following narrative:

I don't feel like many units are making use of that [interpreter hotline]. It's just every unit has their own thing, right. Mental health is booking interpreters and [medical oncology] makes use of this line [interpreter hotline] and a lot of other units are good at utilizing the little cheat sheets with the language. 
Three of the four nurses who rotated to a mental health unit spoke about the use of inperson interpreters in the mental health unit and units such as oncology, where the psychosocial dimension is heightened. However, the use of in-person interpreters was minimized in other units they worked in such as surgical and medical service areas. NRT 4 and NRT 5 discuss the use of interpretation services in oncology and psychiatry where the emotional and psychological dimensions are explored more:

Um-m-m, I think some units are better at utilizing the resources, I'm thinking [medical oncology] like that one in particular. I know they utilize the phone translation services. They're a lot better at accessing [the hospital's] services and I guess there's a lot of need for emotional support and that there. So, I think that floor does it the best. . . Y Yeah, it seems that's when the resources are used more, when the psychosocial and emotional dimension is explored. (NRT 4)

When we do have translators in the weekdays where we're able to book translators that's what the psychiatric team uses to do their assessments. So, just like a room, like this, we'll have a translator on one side, patient on one and the person doing the assessment, so there's a big reliance on translators. (NRT 5)

In addition, when the emphasis is on the psychological and psychosocial aspects of patient care, the need for accessing interpreter resources is utilized to a greater degree. However, interestingly nurses spoke about the importance of the subjective element in assessment regardless of service area. As NRT 4 explains:

Very significant. Like I said it [language] relates to a lot of things really, because it's how you find out how they [patients] are doing psychologically. It's a whole other aspect of 
nursing. It [language] can prevent errors just by having the patient being able to say their story or say what they need. ... It's like a safety thing too.

Most of the nurses stated that they have no knowledge of where to access and how to use the interpretation resources at their organizations or cannot remember how to use the service, thus creating challenges in finding and using the resources. Take, for example, NRT 6 who was aware of the interpreter services but did not know where these services were located. This nurse exclaimed:

Where do we get that? [facial scales to measure patient's subjective pain experience]. I don't see it [facial scales] available anywhere on the floor. Like it's all these objective tools. I know I learned about it [facial scales] but like I don't see it anywhere.

Overall, nurses mentioned the lack of use of the interpretation resources across all units and stated that they haven't seen many nurses use these organizational resources. Along the same line, NRT 5 discusses the uncertainty in the uptake of the interpretation services for direct patient service:

I don't know what context it's [interpreter portable phone] being used, if it's even being used. But being a front line person [nurse], ah, providing care, I've never been told about it or like I [have] never seen any of my colleagues use it.

NRT 5 goes on by speaking about the invisibility of interpreter services on some units while observing in-person interpreters on psychiatry units by saying, "I haven't seen any translators on the general medicine or surgical side. It's just in the psych[iatry] side I actually [have] seen them hire a translator to come in."

Nurses voiced a need for training on the available organizational resources and wanted this training to be inclusive and reflective of the various languages spoken by their patients. Nurses 
continued by saying that the need for training should include (a) where to access resources, (b) the various types of resources and, (c) how to use each resource. Three of the eight nurses received organizational training on the mobile phone interpreter services whereas the remainder of the nurses expressed the need for this type of training. These nurses became aware of organizational resources through their experiences across units as well as their own observations. As NRT 5 exclaimed, “There's no training, it's [interpretive mobile phone] just there. I've seen it from my observations ... I've seen something like catch my eye once.” The nurses also became aware of organizational resources through their collegial interactions on various units, "Um, like my time on the medical oncology [unit] is what introduced me to this interpreter line" (NRT 4). The following narrative by NRT 4 explains the need for "some sort of training" regarding available interpreter services: "I think it would've been . . . very useful to have some sort of training. I know that [the hospital] has, um, some sort of interpreter telephone service but I don't think very many people know how to access it or use it.”

NRT 4 continued by saying:

We live in [an urban city], it's a multicultural city like most people . . your patient load in a day, how many will speak English fluently? I'm sure at least half are speaking some other language, right. So, I think having that training is important and having more research and studies like this done is important for sure, especially in this city.

NRT 4 also discussed the need for various training methods as language diversity and thus language barriers are common by saying, "it would kind of be nice ... if our hospital provided us with different ways in training . . because it's [language barrier] very common." Moreover, nurses mentioned the need for continued professional development to ensure effective and efficient use of the interpreter services. This need for continued professional development is 
needed as the nurses who had training stated that the resources were not user friendly. As NRT 3 explained, training is a continual and ongoing process of learning and not a one-time session: They did train us [nurses]. They did train us when they brought them [interpreter devices]. I remember this was a few years ago. They did train us but that was it. Like it ended right there. After a few months, if you haven't used it [interpreter device], you forget.

\section{Lived Time}

The experiences of the nurses were situated in lived time and actual physical time. According to Mackey (2005), lived time grounds our awareness of our Being. Our temporality signifies the awareness of time through the experience of being in time (Heidegger, 1962 as cited in Mackey, 2005), and as something perceived (Caldas \& Berterö, 2012). Furthermore, clock time is measured (Caldas \& Berterö, 2012; Mackey, 2005) and serves as a form of shared time that is used to organize daily life (Mackey, 2005).

\section{Everything Comes Down to Time}

The temporality of their role as a nurse on the NRT emerged as everything comes down to time. Nurses highlighted the significance of time within their space in healthcare as, "In healthcare, time is really, really crucial. Everything comes down to time" (NRT 3) and "time is something where it was very limited for us" (NRT 6). The experience of time was expressed as limited and not enough to match what care needed to be completed to ensure quality of care when a language barrier existed. The subthemes that emerged from everything comes down to time were (a) feeling the pressure of time (b) lack of timely care and (c) taking the time to translate. 
Feeling the pressure of time. Nurses in this study talked about the pressures regarding the nature of their nursing job as experiencing "time constraints". Time constraints were discussed in light of the varying responsibilities required of them to ensure the needs of all their patients were met within a reasonable timeframe. NRT 1 commented:

There's a certain number of things to do and not a lot of time to do it. And just like don't really see the issue the person is having. And could be a bit of like "no, I need you to do this" and just proceeding.

This above excerpt by NRT 1 referred to the juggling act of meeting all of the patient's needs within a tight timeline to the point where the holistic understanding of the patient may be missed or minimized. Similarly, NRT 2 speaks about this pressure of time impeding the ability to provide care:

In terms of actual practice, like I try to give them [patients who speak a different language from the nurse] as much time as I can. But if I'm spending longer than I would like to, I kind of would have to leave the situation and come back to them especially if an antibiotic is due or if another patient needs to go for a procedure or if they're calling me at the front desk. Like I can’t . . like I would like to give them as much time as I can. But just time constraints.

NRT 2 continues by saying:

If I had more time, like all the time in the world and all the resources in the world I would pull up the [interpreter] machine and it would be so much easier but they [organization] don't really allot for that. They [organization] don't allot for that.

Another nurse, NRT 3, discussed the limits to their abilities within the allotted time thus further feeling the constraints of time when trying to understand the needs of a patient who does 
not speak English or French. NRT 3 explains that nurses would rather figure out what the patient requires by asserting:

With time constraints, nobody wants to go find where it [interpreter device] is and then you have to figure out how it [interpreter device] works. Because we don't really use them [interpreter devices]. All of that time constraints, a nurse would rather go and try to figure out what a person is saying.

Lack of timely care. Within the context of lack of timely care, the importance of shared meaning was mentioned by nurses as a tool to save lives. The importance of the immediate nature of knowing about matters that were time sensitive such as acute changes to patient health, preparing patients for diagnostic tests and timely discharges were discussed by the nurses. As is clear in the following passage by NRT 1, the immediacy of knowing patient concerns is medically significant in providing care in a timely manner:

I'm sure there have been times when there have been delays when it's been medically significant ... and then half an hour later, we caught the answer, "it's chest pain!" [laughing]. It's like maybe if we had known that sooner the outcome can be different. (NRT 1)

Both NRT 1 and NRT 4 discussed the delay in care that resulted when a barrier to communication between the nurse and patient exists. NRT 1 found that the delay in timely care was a result of the delay in completing the patient assessment due to a language barrier. In the following excerpt, NRT 4 shared that the delay in sending the patient to diagnostic imaging was because of a language barrier in combination with a patient experiencing confusion. In this instance, the lack of timely care was heightened. 
A patient going for some testing was resistant and had a language barrier. That was very difficult because ... when you try to convince someone who is a little bit confused, um, and try and explain to them the reasoning, um, "What's the importance of this test", "Why you need this", "What we're doing to you" and even something as simple as inserting an IV. Like this person can be consoled if they know what's going on but the thing is when one is a little bit confused and doesn't understand English it becomes worse. So I think that was definitely one of the most challenging shifts I've had. . . I I believed the patient got it [understood] but after a long, very time-consuming process. So, I think it [language barrier] definitely can delay care sometimes.

Taking the time to translate. In addition to feeling the pressure of time, the time to use the interpreter services was considered, by the nurses, to be lengthy and not conducive to the job structure. Hence most nurses utilized Google Translate for convenience. Even nurses, with organizational based interpreter resources, often did not use the interpreter resources due to the time required. As NRT 2 explained, "I've rarely seen a nurse have time to use the [interpreter] machine and like personally I don't have time either."

The length of time needed to use the organizational resources was considered to not be conducive to the acute care setting. Nurses stated that the set-up process required time that was not really possible given the current work environment. As NRT 5 shared, "I feel the hassle of having to take the phone there. It's just in the day-to-day busyness of working on the floor. It's just not something that's practical." Similar to NRT 5, NRT 8 said, "Honestly, I've never really seen any nurses using it [mobile interpreter phone]. I don't know if cause there's no time to use it or they don't even bother." NRT 2 agreed by adding, "Some units have the [interpretation device] but sometimes it's like I'm ... time crunched and I have a billion things to do." 
The acuity of the patient is a consideration when deciding whether to use the translation equipment or not as discussed by NRT 1. "Um, when it comes to non-life or death things, people are not always reaching for the translation stuff. It does take time." Similarly, NRT 3 talked about interpreter devices being used in "real cases [incidences of high urgency]" as the set-up time is a deciding factor:

But I do remember them [interpreter device] when they brought them and I don't know . . . they [nurses] only use them in very real cases [incidences of high urgency], when they really need [them]. I heard in emerg[ency] they use them. In real cases as well. Because it takes time to set that up, with everything.

The amount of time required to set up the interpretation service depended on the need for in-person versus over the telephone interpreter as well as the language the patient spoke. Inperson interpreters required time to initially schedule as discussed by NRT 1 :

Yeah, they [interpretation service] generally are quite accessible. You sometimes need an appointment. The telephone ones are much quicker, um, where you could in like 10 minutes be on the phone with someone who will offer translation. Um, like the Albanian lady, I think it took twenty-four hours to set up, but once they [healthcare team] did, they just scheduled it every morning for the foreseeable future until they were ready to cancel it.

The nurses appreciated the immediacy of the mobile service app, Google Translate, which was a method the nurses found conducive to their workload and work environment. For example, NRT 6, in the excerpt below, discussed their use of Google Translate within the temporal context of nursing: 
Um, for me it's a quick easy tool, um, and easily accessible. Um, and it's very important especially when I work on the floor, 4 to 5 patients you have look after. You just want like a quick assessment like, “Okay, there's something I could do for this patient right now." Um, so I think that's very important because honestly like time is something where it was like very limited for us, um, when we used to work on the floor, um, and you just want to see what are things we could address right away ... So, um, it's obviously very convenient, that's the thing with Google Translate.

NRT 6's excerpt illuminates the need for a translation service that was quick and convenient and helped with addressing patient needs. Nurses had differing opinions regarding the additional time needed for patients where a language barrier existed. For example, NRT 3 spoke about the need to provide additional time to ensure equitable care was provided:

They [patients] definitely need your extra time cause it needs patience to work with patients like that, especially in fast paced [units]. They need your time, they need your advocacy because they wouldn't be able to express what they need.

NRT 6 found that the extra time needed to understand patient needs within the context of a language barrier added to the workload:

Yeah, it's just like, especially on the floor, it's heavy, you get so many patients. And the language barrier, in a way it's adding to our workload, right because we have to go through all these questions, and trial and error, translation, getting someone to help translate. It takes time because your time is so limited and there is only so much you can do. 
Interestingly, assistance from family members that were physically present meant less time was needed to plan patient care. However, when families were contacted, the experience was felt as taking up a "great deal of time" as discussed by NRT 4 :

If you need to call a family member to help translate, that takes a great deal of time that you may not necessarily have. So I feel like all that extra effort . . can take away from the care either to that patient or to other patients.

Thus, the temporal experience of nurses caring for patients when language barriers exists grounds the nature of their work and workload.

\section{Lived Human Relations}

Lived Human Relations (relationality or communality) involves the interpersonal space individuals share with others (van Manen, 1997). According to van Manen (1997), the pursuit for a shared relation is sought by humans to achieve meaning and purpose. The experiences of the nurses, in this study, with patients who did not share the same language were dependent on how they interacted with each other. The relational theme that emerged as essential to the nurses' experience of caring for patients when a language barrier exists is desiring shared meaning.

\section{Desiring Shared Meaning}

The aim for nurses in this study was to achieve two-way communication in order to better understand the patients' needs:

Being able to complete assessments you need to have a two-way communication with the patient, you're not speaking one way towards the patient, right, like we need to get feedback and find out about pain, about symptoms and like their past medical history so, it's a huge part in the communication. (NRT 5) 
Communication in the nurse-patient relationship is not unilateral but one that is bidirectional to meet patient needs by co-creating shared meaning. For the nurses, desiring shared meaning was a significant element of their experiences as they longed for shared meaning with their patients. The subthemes that emerged from desiring shared meaning were (a) building emotional connections, (b) expressing compassion and empathy, and (c) resorting to reductive relations.

Building emotional connections. Most nurses spoke about challenges in building an emotional connection and rapport with their patients due to being an NRT nurse. Not being able to care for the same patients for a duration of time caused the nurses to miss out on the opportunity to co-create routine with the patient, build rapport with the patient, and develop an emotional connection with the patient. In contrast to unit based nurses who seem to be able to build rapport while caring for patients when a language barrier exists, NRT 6 discussed the challenges of building rapport due to the nature of the NRT nurse role:

Just because you're at different areas like everyday. There's not much of a rapport with the patient you met versus if you're on the same floor. You have probably a better understanding, a better rapport with that patient if you ... don't speak that language. Yeah so I think that's how I feel about being on NRT and have the whole language barrier.

Similarly, NRT 1 discusses the nature of being an NRT nurse and the lack of the opportunity in developing a connection:

You know things are busy. And ... there is lots of little interactions that go into like building a rapport. And I only have one day of little interaction, so it tends to be pleasant but not very deep in terms of a connection. 
The NRT nurses relied on their nursing colleagues to assist them by supplementing their knowledge of the patient with additional helpful information. As NRT 6 states, "we do have somewhat diverse colleagues, like nurses, at the hospital so it's always good to ask one of the coworkers to help." NRT 6 continues by saying,

I guess utilize my resources like, really have to ask my co-workers. I don't like asking for help if I don't have to cause usually ... I know everyone is busy with their work, um, but yeah, really just have to ask your co-workers for help at times if no one is around, if no family or friends are around to interpret.

The nurses found that this additional information assisted them in developing a connection with their patients as reflected in NRT 8 excerpt of how interpretation by colleagues was helpful, "Yes, and that helped a lot. It helped too.... I would use another nurse". NRT 6 discusses the challenges of being an NRT nurse and finding that caring for patients when a language barrier exists made it more challenging to build rapport and make emotional connections with their patients. The following excerpt from NRT 6 discusses this difficulty with establishing rapport and shared meaning as an NRT nurse:

NRT is definitely more challenging especially if you're working, say you're working on the same floor, you have that patient for like few days in a row and you sort of recognize the pattern of behaviour and like what the patient wants and the family comes in and [you] get to ... know the patient better. It's a bit easier. Whereas in NRT, we go to a different floor every day. And it's like a different patient and with like additional language barriers, just a little bit more difficultly because we first have to learn about the patient first. But with a language barrier, it'll be a little bit even more difficult. But like I said, if you are on the same floor, it's a little bit easier because you kind of get to know 
the patient better throughout the days. And, um, if you have the chance to speak to the family you get to understand the patient's history and ... it's a little bit easier to manage rather than if you were a float [NRT nurse]. I think that's the main difference between the two. That's how I felt with being in NRT, versus say if you were a regular floor nurse for that patient.

Expressing compassion and empathy. The majority of the participants voiced empathy and compassion for the patient in navigating a system where it is a challenge for the patient to voice their needs when a language barrier is present. The empathy and compassion the nurses felt was heighten when they saw the patient upset and/or passive of their desired needs. The following accounts exemplify this emotive feeling of empathy and compassion the nurses felt towards their patients. In this quote, NRT 1 shared how they thought the patient was feeling: Waiting for test results where like no news is good news, but it can be stressful waiting, um, and when you don't have the ability to have those small conversations with your nurse like [mimicking voice], "Is this normal?". . I would think it would be stressful because from my point of view you are doing well, have no concerns [laughing] but you don't know that, so that would be very stressful.

NRT 3 also spoke about how she felt empathy towards her patient by stating, "But at the same time you feel empathy because you don't know what to do to help this patient that you wish you could do, you know, so you try to find other ways that you could help." Similar to NRT 3, NRT 5 also spoke about her feelings when caring for patients when a language barrier exists: It's [language] a very important dimension of the care we provide .... They [patients] could feel powerless or feel like they're alone or having to wait on family to come and 
like I feel bad for them too cause they're in a vulnerable situation. They're in this big hospital where they're all alone.

In keeping with the above excerpts, NRT 7 spoke about their feelings for patients when a language barrier existed: “Compassion or empathy and just feeling for this person who can't actually say what he or she wants to say in a way that I could understand it."

Moreover, most nurses experienced amplified feelings of empathy when they witnessed patients becoming passive and giving up on something they wanted. NRT 1 talked about what happened when patients knew they were not understood, "because sometimes when people [patients] know they're not understood they'll just be very passive and kind of let you do whatever and I find it a little sad." NRT 8 shared a similar situation where the patient did not feel understood, "Sometimes they just give up, and they kind of just like wave at you like, 'forget about it.' For me, sometimes I feel like I want to do more for the patient."

One nurse (NRT 7) talked about how tension turned to compassion or empathy when reflecting on their own parents' background:

So, there is sometimes a sense of tension but I'm from parents where English isn't their first language. So when that happens there's also a sense of feeling of either like ... compassion or empathy and just feeling for this person who can't actually say what he or she wants to say in a way that I could understand it.

Resorting to reductive relations. Nurses resorted to reductive relations in engaging with patients where a language barrier was present. Desiring a shared meaning led nurses to resort to these reductive relations. These reductive relations comprised gestures, facial expressions, as well as words or phrases that were "simple" and "basic". 
Participants used gestures as a primary means of communicating directly with the patient. The type of gesture used was assumed to be universal and easy to understand. NRT 2 discussed making, "a fake grimace, um, mostly just gestures. Like this is a very easy gesture if I reposition them [patient] in bed, I'll be like, 'Good' [thumb up] or 'Bad' [thumb down] and they kind of communicate with me that way." The assessment of patients was reduced to the physical assessment and physical tasks with less exploration of the patients' cognitive and subjective dimensions. The nuances of cultural positioning were discussed by some nurses as influencing the interpretation of their gestures. NRT 7 discusses the misinterpretation that can occur due to the difference in culture, "So, again. . . going to non-verbal cues, but then there's a chance that it can be misinterpreted based on even like culture.

Nurses also spoke about being attentive to how they presented themselves as they wanted to carry themselves in a welcoming manner. The nurses considered their body language as a means of communication and, therefore, influential to the interpretation process. For example, NRT 5 discussed trying to use "a lot of non-verbal communication ... with my face. If I'm smiling, um, yeah just the way I present myself. I come in with like a nice attitude.”

In addition, nurses were aware of the type of words that were easily expressed to and understood by patients and usually used between 1-2 simple words. Therefore, nurses interacted with patients with simplicity in their choice and amount of words as evidenced by, "I won't always try to ask them very ... long questions. I would just ask them 1 to 2 word questions. Like very simple phrases" (NRT 6). Interactions with patients also relied mainly on auditory and visual cues. Nurses did not generally engage in what may have been considered "complex" interactions. The nursing care became limited as the nurse-patient interaction was simple and not comprehensive. Two of the nurses even found that they resorted to speaking to the patient in a 
manner that could be considered "child-like" (NRT 1). Similar to NRT 1, another nurse (NRT 3) found that she spoke to the patient like a child in order to gain a better understanding of their needs. Both these nurses demonstrated a desire for a shared meaning between themselves and their patients.

Kind of like you are talking to a child. Not that you are making the patient as the child, but the child who does not have the language to speak to you. There's ways we can speak to them to understand what they want. (NRT 3)

NRT 1 further noted the similarities in speaking with a patient when a language barrier exists to how a nurse interacts with a patient who is experiencing confusion. Both methods of communication were simple and focused on the patient's basic needs:

Like treating people like their children. It's an issue with dementia in general where they can have child-like behaviours, and interests and abilities. So then people start treating them like children and they are not. . . But it's, it's definitely worse with a language barrier. If you kind of get what you're communicating really simply, and you're focusing on basic needs and you are kind of treating them [when a language barrier exists] like confused people [tone softens]. . . . Treating people like their children just because you are trying to keep things simple and clear and easily understood.

In the quest for a shared meaning, most nurses spoke about the creation and/or utilization of a co-created communication board with family. This communication board was usually initiated for the patient by HCPs to communicate commonly used words or phrases. NRT 2 described what this communication board looked like: "It's like, created piece of paper, laminated, or literally on the ... white board . . you put your name, date, whatever, and then 
they [nurses, family and/or healthcare team] would just write simple phrases." Another nurse described how the communication board was used:

What we usually do, ask the family when they come in to tell us some . . words, few things we deal with every day. ... So, they [family] give us the word and we put a paper on the wall for a few of the words, like, "thirsty", "pain", or maybe "I need this" . . so we know. When we go to the patient, and they are probably talking or screaming, we look at the notes, what the patient is trying to say. That's one way we try to. . . get an understanding. (NRT 3)

Nurses also stated they remembered certain words commonly used in their practice that they could use for their other patients when a language barrier exists. In the following excerpt, from NRT 5, the use of gestures and words in the patient's language are used simultaneously in an attempt to elicit patient feedback:

For example, I've learned how to say "pain" in a couple different languages like in Italian, "dolore". I'll go up to an Italian grandpa and ask them if they got "dolore" and like point at whatever . . . if they had a hip replacement . . . and they'll understand what I'm saying. But I think it's just a matter of having, um, some of these key words if you pick up on them.

Similarly, NRT 8 talked about remembering language specific words that assists with delivery of care. However, both excerpts illustrate the simplicity of the words that lead to limited feedback from patients; therefore, elaboration from the patient is minimized.

I would try to remember the specific words for that language, so that when I do encounter like another Italian speaking patient, I'll recall those words that I used and it'll help me be able to communicate with them if they don't use any English what so ever. So, I try to 
remember those words for the other patients so hopefully I could provide better care or understand how they're feeling or what they need even if it's basics of like water say, "acqua."

\section{Lived Body}

Lived body refers to "how the body is experienced with respect to the phenomenon that is being studied" (Manen, 2014, p. 304). According to van Manen (1997), we meet others in their lifeworld, first through a bodily presence. As the role of a nurse involves a bodily experience, the nurses' experiences of interacting with patients who they did not share the same language caused the nurses to experience disequilibrium.

\section{Experiencing Disequilibrium}

On one hand, the nurses felt frustration in their interactions with patients where a language barrier was present. On the other hand, in order to work with diverse kinds of patients, the nurses realized that they needed an "open mind", needed to be "creative", be "quick on the feet" and "learn patience, adaptability and flexibility." Therefore, the subthemes that were exemplified in experiencing disequilibrium were feeling frustrated and embodying skills that facilitate care encounters.

Feeling frustrated. Participants expressed frustration in their interactions with patients where a language barrier was present. NRT 7 captures the feelings of frustration by sharing, "I've heard and seen things, um, where a lot of that frustration is because no one is speaking English.” NRT 3 added that feelings of frustration occurred when not being able to assist with patient needs by exclaiming, "It's really frustrating ... that you cannot help the patient. .. . You cannot understand." This feeling of frustration was heightened due to their role as an NRT nurse. 
In the excerpt below, NRT 1 discusses experiencing frustration when a language barrier prevented an understanding of the patient's needs:

Like "I don't understand you [patient]", and "I want to" and "I'm trying" [laughing]. It's just frustrating. And like you [unit-based nurses] could get to know someone [patient] and their patterns and behaviours. And when floating [working as an NRT nurse] you really can't. Like you start from scratch every day ... And it's frustrating for everyone because they [patient] want to be understood. You want to understand them.

NRT 1 continued by saying, "But sometimes it takes effort not to be annoyed with the person for not being able to communicate basic things to me. .. . Like I can’t speak every language. And I would love to." NRT 1 further reflected on the importance of inclusion and their own personal feelings of feeling frustrated:

The basic food and drink words, I don't know. I just want to give everyone little travel books like basic phrases you might like, "Where's the bathroom?”, "I need assistance” . . . you know just handy phrases. Like a lot of the times . . . it is frustrating. . . It's not wrong to speak your own language and live your own culture. I don't like that I do feel frustrated.

In addition, frustration also stemmed from facilitating the interaction where a barrier to language was present within constraints of time. As NRT 6 pointed out, “if a patient doesn't speak my language ... it's really frustrating at times cause you're really time constrained especially on the floors, sometimes you can't really have like a full conversation like we're having just like back and forth." As discussed by NRT 6, the process to translate requires time and the added layer of time constraints led to experiences of frustration. 
Embodying skills that facilitate care encounters. Because of their role as NRT nurses, the nurses quickly realized that in order to care for their patients when a language barrier exists, they needed an open mind and needed to be "creative and flexible". As one nurse (NRT 3) explained:

I don't know what to expect. I really don't know what to expect. So I just go there [unit] with an open mind. You just have to be creative. You learn as you go and you become creative, cause every unit is different, every team you're working with is different. Um, so you become creative, you become flexible, you learn how to work with, um, diverse kinds of patients with diverse kinds of nurses as well. Cause some [nurses] will be helpful and some will not be helpful, even if you have patients that don't speak the language. You might have a team that is not helpful, so you cannot rely on that. You just have to . . . learn on the go, really. So it kind of forces you to be creative, to be creative and flexible, cause you don't know what to expect.

Because of working on the NRT, NRT 6 discussed the need to acquire a skillset that allowed them to work with very diverse patient populations:

I guess for NRT .... it really helps you to ... train to become a more flexible person ... flexible in terms of opens up your mind to all these people of different backgrounds, um, and just learn ... teaches you to be a person that will have to adapt to different situations, any kind of situation, any kind of persons of different backgrounds. And . . . it kind of gives you like, um, idea of what things, like the tools you think would work. . . So NRT ... gives you like diversity and makes you think quick on the feet with all these patients of different populations. 
Similarly, NRT 7 discussed skills developed by NRT nurses in order to care for their patients when a language barrier exists that may not necessarily be needed by unit based nurses: As float poolers [NRT nurses], you got to learn patience, and adaptability and flexibility, things that nurses on a floor who work on that same floor don't necessarily need. So, as a float pooler, being able .... to use those skills and to ... have a situation where I can't either get through or there's a break in communication, using that ability to be able to adapt and be like, "Okay, so I can't get to him this way. How can I get to him the other way?" ... . I think that as a float pooler we have those toolsets already and it makes it a little bit easier maybe to deal with language discordance.

\section{Summary}

In completing this thematic analysis, a deeper understanding of the experiences of resource team nurses experiencing a language barrier has been achieved. In the pursuit to understanding, nurses are trying to span the distance between themselves and their patients by playing guessing games, finding and using resources, and by just muddling through. They desire a shared meaning with their patients by building emotional connections, expressing compassion and empathy, and resorting to reductive relations. However, the nurses found that everything comes down to time which caused the nurses to feel the pressure of time, caused a lack of timely care, including taking the time to translate. The nurses experienced disequilibrium as evidenced by their voiced feelings of frustration but also in their ability of embodying skills that facilitate care encounters when a language barrier exists.

Chapter 5 will present the discussion of the findings with incorporated literature. Also, the implications and lessons learned from the field of this present study are discussed. 


\section{CHAPTER 5: Discussion and Implications}

\section{Introduction}

In this final chapter, I compare and contrast the findings of this study with relevant literature to interpret and deepen our understanding of NRT nurses' experiences of caring for patients when a language barrier exists. The overarching theme and four essential themes, from the previous chapter (See Chapter 4), are presented using the structure of the four existentials, namely, Lived Space, Lived Time, Lived Human Relations, and Lived Body. I then present the implications of this study for policy, practice and further research. Subsequently, I discuss lessons learned from the field and provide a concluding discussion.

\section{Overarching Theme: A Dialectical Experience in the Pursuit to Understanding}

Nurses' experience of understanding patients was dialectical. Nurses' desired emotional connection and shared meaning while the relational distance challenged their ability to connect with patients. The dialectical experience was shaped by the nurses' position of being an NRT nurse.

The desire to communicate is a fundamental need between nurse and patient (Coleman \& Angosta, 2017), and for nurses in this study language emerged as a medium to discover and meet patient needs. Language, according to Heidegger, is how Being is unveiled and existence is communicated through language (Langdridge, 2007). Language allows for inter-subjective experiences to be produced and expressed (van Manen, 1997), thus it is an expressive medium (van Manen, 2014). Moreover, language describes what is experienceable leading to inner experiences being discovered (van Manen, 1997). Therefore, when a language barrier exists the inability to connect with patients relationally, as is expected in nursing practice, presented 
challenges for the nurses. Finding it difficult to uncover the patient experience lead the nurses to describe nurse-patient interactions as challenging, time consuming, and frustrating.

Nurses in the study found that language is shaped by culture, and therefore even when language is available as a tool to connect, there are cultural undertones and nuances to the meaning of language that may be missed when trying to understand the patient. Even when language barriers were present, nurses sought other ways to communicate with patients such as non-verbal cues and gestures. The nurses also used simple, basic, phrases. In this context, communication extended beyond language and served as an important feature of connectedness.

\section{Lived Space: Trying to Span the Distance}

Lived space grounds human beings in a locale (Mackey, 2005) and according to van Manen (1997), space affects the experiences of humans. As individuals we are not solely a cocooned body but rather a "dynamic manifold of reaching and spanning, which in advance opens up and encompasses the environment through which we move" (Eiland, 1984, p. 45). The spatiality of the nurses in this study was exemplified in the relational distance with patients and in the available resources in the presence of a language barrier. The ability to limit the relational distance between themselves and the patient was challenging causing the nurses to explore various options to establish shared meaning.

Nurses in this study attempted to span the relational distance through the use of mobile apps such as Google Translate or by relying on their colleagues and the patients' families and friends. Interestingly, nurses considered mobile apps such as Google Translate to be convenient in lessening the relational distance between patients and themselves. However, concerns with accuracy of information and lack of in-depth inquiry was also discussed by the nurses. Patil and Davies (2014) speak to Google Translate being easily accessible, however they found significant 
errors with health related inquiries and argue this app should only be used if professional interpretive services have been exhausted and the situation is clinically important. The dependence on colleagues, patients' families, and patients' friends is consistent with current literature where in healthcare spaces when there is a language barrier, interpretation is often done through non-formal untrained individuals (family, friends, HCPs and non-clinical staff), who are referred to as ad-hoc interpreters (Brisset et al., 2014; Edwards et al., 2005; Probst \& Imhof, 2016; Ramirez et al., 2008). Nurses, in this present study, found working with families as interpreters was complex. At times, the nurses experienced confusion regarding what the families were conveying compared to what they observed or perceived with their patient assessments. This complexity when working with families is similar to present literature (Meddings \& HaithCooper, 2008), whereby concerns with accuracy in information exchange arose with ad-hoc interpreters

On one hand, in the nurses' attempt to bridge the relational distance in language through the use of these above means, they inadvertently and simultaneously widened the distance by placing barriers between themselves and the patients by limiting and interrupting their care and questioning the accuracy of the care they provided. However, these tools also served to facilitate the nurses' care when the tools yielded pertinent information from the patients.

When ad-hoc interpreters were not present, nurses engaged in a guessing game with patients. The guessing game in this study referred to the back and forth dialogical experience, either with the use of a few words or actions/gestures, between nurse and patient in the pursuit to identify, validate and address the concern of the patient. Though relatively unexplored in the literature, the nurses in this study seemed to have a shared understanding that the process of the guessing game was filled with uncertainty. Thus, the care the nurses could provide and what 
patients could receive became reduced to guesses and assumptions. It is problematic for nurses to rely on guesses or assumptions in the provision of safe and quality care, as misunderstandings and errors can occur. However, when nurses and patients came to an agreement of what the need was, nurses experienced relief.

Challenges with finding and accessing organizational resources were discussed in the literature (Brisset et al., 2014; Coleman \& Angosta, 2017; Higginbottom et al., 2015; Ian et al., 2016; Kale \& Syed, 2010). Specifically, in Brisset et al.’s (2014) study, the majority of the practitioners were not trained to work with interpreters and often did not bother to contact professional interpreters. The participants in Brisset et al.'s (2014) study reported reasons for not contacting professional interpreters such as being uninformed of available linguistic resources and the procedural processes required to access these resources. Similarly, nurses in this present study expressed the need for more organizational supports through easily accessible resources, training and continued professional development services. Without these organizational supports and resources the nurses were forced to find alternatives like Google Translate, patients' families, patients' friends, and colleagues. But, there were still occasions where the nurses had to resort to guessing and making assumptions which could lead to safety issues.

Ian et al.'s (2016) study on the experiences of nurses with language barriers discussed the importance for healthcare organizations to assess and evaluate their available current supports and resources to improve care delivery for vulnerable patients. In this present study, most nurses attributed cost as a potential contributing factor to the limited availability or lack of interpreter services at their organizations. The costs associated with interpreter services were discussed by most nurses in this present study. The costs related to interpretive services were also discussed in the literature as a systemic barrier as these supports are not covered by the provincial healthcare 
plan thus leaving organizations to incur costs within their already limited budgets (McKeary \& Newbold, 2010). However, health outcomes need to be prioritized over costs, as regular use of interpretive services optimizes health outcomes and thus reduces long-term healthcare expenditure (Bischoff \& Denhaerynck, 2010; Brisset et al., 2014).

Due to the nature of their role as NRT nurses, the immediacy of shared language is essential as these nurses float from unit to unit and do not experience continuity of care and do not get to know the patients. Not caring for the same patients over a period of time does not allow the nurses to learn the patterns of behaviour and routines of patients which assists in relational care. Thus, this present study highlighted the inadequacies of organizational supports and systemic barriers that can lead to the delivery of limiting and non-inclusive healthcare, and also highlighted the role of the NRT nurse.

NRT nurses are particularly well positioned to offer insight into where resources should be distributed as they provide care across various units and have knowledge of how language barriers are facilitated in various spaces. The NRT nurses, in this present study, shared that most of their nursing colleagues (i.e. both NRT and unit based nurses) do not utilize organizational resources because of a lack of awareness, lack of training, lack of availability, and practicality issues. However, the units where the NRT nurses found opportunities to lessen the relational distance between themselves and patients were emergency, mental health and oncology as these units were more likely to have access to an interpreter. Similarly, Ian et al. (2016) found that many of the nurses working in the emergency department felt they had institutional supports and resources. In agreement, nurses in this present study stated that it is important that interpreter supports and resources are in place in the emergency department when caring for patients when a language barrier exists. However, the nurses in this present study also suggested that supports 
and resources need to be in place once patients are admitted to floor units as the trajectory of the patient experience does not end in the emergency department for all patients. In keeping with these accounts, the use of interpreter services was discussed by the NRT nurses in this present study who service the oncology unit. Although psychosocial and emotional care is foundational to nursing care, these NRT nurses in oncology attributed the need for interpreter supports and resources to better explore the psychosocial and emotional dimensions of care to enable them to provide additional emotional support when a language barrier exists.

From the discussion with the nurses, in this present study, the importance of the patients' subjective voice was found to be important in all service areas. However, understanding the patients' subjective voice varied depending on the availability and use of resources across various units. Therefore, it appears that the use of resources increases when retrieving subjective data from patients, such as with mental health assessments where the NRT nurses rely on the subjective voice and also in oncology units where nurses focused on eliciting the patients' subjective voice to uncover the emotional and psychosocial meaning of cancer. The nurses in this study did not explicitly acknowledge other units that require language services, however this is not to suggest that other units are not in need. Further exploration related to the type of nursing care and language service needs warrants further investigation and was outside of the scope of this study.

The NRT nurses, in this present study, expressed that the lack of interpreter supports and resources significantly impacted their ability to find shared meaning with patients. As a result of a lack of organizational interpreter supports and resources and the nature of the NRT nurses' job (i.e. floating from unit to unit), nurses resorted to simple, basic, and convenient methods in the pursuit to understanding patients. Although the NRT nurses who worked in academic teaching 
hospitals experienced this lack of organizational supports and resources on units, the lack of supports and resources was more noticeable in community based hospitals where nurses pointed to the lack of resources throughout the organization. This difference in resource availability is reflective to the funding allocation between both types of hospital structures, whereby community hospitals receive lower levels of funding than academic teaching hospitals in Canada (Topps \& Strasser, 2010). Therefore, differences in resource allocation across different organizational settings impacted the facilitation of an interaction where a barrier to language was present.

\section{Lived Time: Everything Comes Down to Time}

The conception of time and how it is experienced is important to consider. In this present study, the NRT nurses emphasized time as being influential to their experiences in caring for patients when language barriers exist. The concept of time is discussed in the literature pertaining to how HCPs and nurses perceive and experience time in the healthcare space (Blackford \& Street, 2002; Brisset et al., 2014; Coleman \& Angosta, 2017; Hendson et al., 2015; Ian et al., 2016). Similarly, in this present study, nurses' temporal experience was such that, everything comes down to time. The lack of or the time needed to access interpreter services was reported by the NRT nurses as impacting the provision of timely care that often resulted in delays to care. Similarly, the lack of interpreter services is cited in McKeary and Newbold's (2010) study as delaying appropriate care, especially when the need is acute in nature, and thus time sensitive. Moreover, the NRT nurses, in this present study, felt the pressures of time in managing their workload and multiple nursing responsibilities in addition to providing additional time for patients when a language barrier existed. The felt pressure of time shaped the nurses' 
experiences of felt time and caused time to occur at a faster pace, which reflects Tuohy et al.'s (2013) statement that lived time is perceived to be sped up when individuals are busy.

NRT nurses in this study also spoke about time constraints and discussed the need for additional time to account for interactions where a language barrier is present in order to provide equitable care. Some nurses conveyed that the required additional time was an increase to workload to which they were already experiencing time constraints. Even in the process of interpreting, nurses commented on the pressure of time, which is also consistent in the literature (Blackford \& Street, 2002; Brisset et al., 2014; Hendson et al., 2015; Ian et al., 2016). In agreement with the nurses in this present study, the need for additional time to provide care is echoed in the literature (Coleman \& Angosta, 2017; Ian et al., 2016; McKeary \& Newbold, 2010). Furthermore, for appropriate care to be provided (Coleman \& Angosta, 2017) that is culturally safe, a commitment to time is needed (McKeary \& Newbold, 2010). Consequently, the lack of available time to care for patients when a language barrier exists reinforces English as the preferred language because English is more efficient and reduces the time needed in care interactions.

Nursing care is often considered as being measured by the clock and thus treated as chronological time (Caldas \& Berterö, 2012). Chronological temporality governs and structures hospitals and institutions (Caldas \& Berterö, 2012) and serves to determine staffing, patient assignment and workload (Jones, 2010). The various tasks and routines are accomplished according to clock time and by a schedule (Caldas \& Berterö, 2012). However, consideration to only clock time can lead to a lack of understanding of nursing care (Caldas \& Berterö, 2012), as time is seen as objective, unmoved by awareness and ascribed the same way for everybody 
(Jones, 2010). Nurses in this present study, found that caring for patients when language barriers exist resulted in not being able to provide equitable care.

According to Jones (2010), regardless of physical or chronological time, if the needs of patients are not met, time is experienced as insufficient. Thus, it is important to know how much time is available for nurses just as it is important to know what care nurses are able to provide and not provide with their allotted time (Jones, 2010). Furthermore, nursing time is important to consider as the coordination and delivery of care is shaped by healthcare organizations' structures of time (Jones, 2010) which situates nurses in unified time schedules that give the structure of practice routine and patterns (Caldas \& Berterö, 2012; Jones 2010). Therefore, time has important "intrinsic and instrumental value in the practice of nursing" (Jones, 2010, p. 193) and thus time needs to be considered in all nurse-patient encounters (Jones, 2010) and in particular when a language barrier is present. In agreement with the literature, the nurses in this present study stated that the time they were provided to care for patients when working within the context of a language barrier was not sufficient to provide timely and culturally safe nursing care. Consequently, the nurses needed to take extra time (not allotted to them) to find ways of translating which only heightened their feelings of the pressure of time.

\section{Lived Human Relations: Desiring Shared Meaning}

The desire to build meaning and make sense of experience is what van Manen (1997) argues is to be human. In this present study, shared meaning exemplified the theme of relationality. Lived human relations (or relationality) refers to how the self is experienced in relation to others with respect to the studied phenomenon (van Manen, 2014). In this present study, NRT nurses voiced their desire of shared meaning in order to enhance their ability to build emotional connections, express compassion and empathy, and resort to reductive relations. This 
sentiment is similar to Coleman and Angosta's (2017) study where nurses expressed the desire to connect and build trusting relationships with patients.

However, the nurses in this present study voiced difficulty in building rapport and trying to build emotional connections with their patients when a language barrier existed due to their role as NRT nurses. The nature of their role as NRT nurses (lacking continuity in patient care as these nurses were not assigned to one unit but floated to various units throughout the hospital) required the immediacy of organizational supports and resources to be able to communicate with their patients when a language barrier existed. This is in keeping with the literature (Higginbottom et al., 2015; Segalowitz \& Kehayia, 2011) who stated that the medium for interpersonal and crosscultural communication is through language. Nurses, in this present study, commented that their unit-based colleagues were able to build rapport with patients through repeated nurse-patient interactions with the same patients whereas NRT nurses were limited with rapport building opportunities as their continuity of care was reduced or non-existent.

Another feature expressed by nurses in this present study was feeling empathy and a sense of compassion for patients who were unable to express their needs. Being with and care are fundamental features of Dasein (Langdridge, 2007; Rentmeester, 2018). Being with (Mitsein) refers to our essence as human beings to be in relation with others (Langdridge, 2007). Care (Sorge) refers to "all being-in-the-world revolves around us being involved in the world, actively engaged in other things and people" (Langdridge, 2007, p. 31). The nurses in this present study spoke about their desire to care, thus experiencing empathy and compassion for their patients but they experienced challenges being with their patients. In agreement with Coleman and Angosta (2017), the nurses in this present study desired a connection with patients and understand patients' needs, thus existing relationally with patient(s). However, this relationality was 
challenging for the nurses in this present study to achieve as there existed a relational distance between themselves and patients when a language barrier existed.

Shared meaning is created by communication that uses verbal, nonverbal and visual symbols (Coleman \& Angosta, 2017). Ian et al. (2016) elaborated by saying that the use of nonverbal skills was considered by nurses as important to facilitating interactions with patients when a language barrier was present. In this present study, the nurse-patient interactions were facilitated by resorting to reductive actions such as by simple, basic, phrases and the use of gestures in the pursuit to understanding. Thus the care became reductive as the measures to which nurses connected with the patient were done through simple and rudimentary methods. The heightened use of gestures in this present study reflects Higginbottom et al.'s (2015) point that non-verbal communication is often more apparent and in situations where language barriers are present this type of communication becomes important. However, the emphasis on nonverbal communication is problematic as according to Segalowitz and Kehayia (2011), patient assessment can became reliant on mechanical and standardized techniques thus minimizing opportunities for tailored, safe and quality care. In this present study, the nurses' use of gestures functioned as a rudimentary communication method in lieu of organizational supplied supports and resources maintaining reductive relations remiss of deeper questioning and understanding.

\section{Lived Body: Experiencing Disequilibrium}

Lived body refers to how the body is experienced regarding the phenomenon of interest (van Manen, 2014). Optimizing the provision of safe and quality care by lessening the relational distance requires an embodied approach. When a language barrier was present, the ability to provide relational care was compromised and thus the nurses' in this present study experienced disequilibrium. The disequilibrium or imbalance experienced by nurses' resulted from the nurses 
feeling frustrated caring for patients when a language barrier existed but also in the fact that the nurses embodied skills (such as being creative and flexible) that facilitated their care encounters.

Nurses, in this present study, expressed feeling frustrated when a barrier to language existed. This finding is reflected in the literature where frustration was expressed when nurses attempted to provide quality care in the context of a language barrier (Coleman \& Angosta, 2017; Hendson et al., 2015; Ian et al., 2016; J. Anderson, et al., 2003). In this present study, frustration stemmed from the nurses not being able to assist with the needs of the patient, constraints with time, in the patient not knowing English, and due to the nature of the NRT nursing role. The frustration that stemmed from the patient not knowing English is problematic as such perception risks excluding patients from their own care and perpetuates Anglocentric care delivery. In Ian et al.'s (2016) study, the weight of the frustration stemmed from the additional time needed to understand patients and in Coleman and Angosta's (2017) study, the frustration arose when nurses were not able to meet the needs of the patient and/or the patient's family. Similarly, in Hendson et al.'s (2015) study, frustration occurred as nurses had the desire to communicate but at the same time felt unable to communicate effectively.

Although the nurses, in this present study, felt frustration they also embraced skills that facilitated their care encounters in the context of a language barrier. As NRT nurses they expressed an uncertainty with what to expect with each patient assignment but embodied an open mind to adapt and be creative, patient, flexible, and be able to think on their feet which facilitated their nurse-patient interactions when a language barrier existed. This is similar to previous literature of NRT nurses needing to be flexible (Hemann \& Davidson, 2012), and adaptable, organized and focused (Lebanik \& Britt, 2015). Thus, nurses in this present study spoke to this 
embodiment of skills and how instrumental the skills were in facilitation of care when a language barrier was present.

Resource team nurses bring forth an array of skills and experiences across a variety of specialty areas that allow for these nurses to be aware of varying unit cultures and organizational processes. These resource team nurses become sources of knowledge and channels of quality nursing practice (Lebanik \& Britt, 2015). By navigating throughout the organization, these nurses interact with various HCPs and have opportunities to fulfill such roles as advocators, change agents, and gatekeepers ${ }^{8}$ (Lebanik \& Britt, 2015). Since resource team nurses are required to work across different specialty areas and at times in more than one setting, they need to be flexible (Hemann \& Davidson, 2012), organized, focused (Lebanik \& Britt, 2015), adaptable, skilled at communication (Lebanik \& Britt, 2015), and have a heightened focus on learning more about the patient, all which serve to differentiate nursing resource team nurses from unit based nurses (Lebanik \& Britt, 2015). Lebanik and Britt (2015) also found that nursing resource team nurses expressed the need for immediacy in understanding their patients and unit protocols (Lebanik \& Britt, 2015). Thus, the immediacy to shared language and meaning becomes significant for NRT nurses and thus becomes challenging with systemic inadequacies that hinder the provision of safe and quality care.

\section{Implications}

The findings from this present study have implications for patient care and safety. This study has shed light on both the NRT nurses' experiences of language barriers as well as systemic concerns, organizational resources and nursing job structures that shape the experience. Throughout this study, a careful consideration of language barrier as an ethical concern was

\footnotetext{
${ }^{8}$ Resource nurses fulfill the role of gatekeepers, which is linking a patient with the most optimal staff and resources (Lebanik \& Britt, 2015).
} 
undertaken to illustrate how language functions within systems such as that of healthcare. The findings of this study will serve to further knowledge as well as catalyze further research. The following implications are presented with recommendations for (a) policy, (b) practice, and (c) future research.

\section{Policy}

The insights from nurses brought forth a deepened and enriched understanding of language barriers within care encounters. The unique role of NRT nurses in caring for patients when a language barrier exists has shed light on the challenges these nurses face throughout the many units they are assigned to. Therefore, the results of this study have important implications for healthcare and organizational policy.

Support for linguistic health services. Nurses, in this present study, found that there was a lack of interpreter resources and resources that were available were not practical to use. The financial support and allocation of funds for hospital interpreter resources needs to be reevaluated by all levels of government to ensure sufficient funds are available and funds are equitably distributed between academic teaching and community hospitals. Costs related to interpreter services are not covered by the provincial healthcare plans in Canada, leaving costs to agencies and organizations (McKeary \& Newbold, 2010). Provincial agencies, regional health authorities and individual health establishments deliver interpretation services throughout Canada (Sultana, Aery, Kumar, \& Laher, 2018). Therefore, the allocation of resources will differ depending on the organizations (Sultana et al., 2018). As noted in this present study, NRT nurses based in community hospitals cited a significant lack of supports and resources as compared to the NRT nurses based in academic teaching hospitals. There are significant differences throughout Canadian jurisdictions suggesting the variability with linguistic needs and distinctive 
divisions among jurisdictions (Sultana et al., 2018). Overall, there is limited centralized or coordinated interpretive services (Sultana et al., 2018). Thus, budgets for interpretive supports, resources and services need to be addressed by the provincial government in order to support linguistic inclusivity in healthcare delivery. According to Coleman and Angosta (2017), establishing and allocating resources is necessary to facilitate care encounters within the context of language barriers in order to lessen medical errors, decrease visits to the emergency department, and increase safety and quality of patient care.

The concern with financial support was discussed by most nurses in this present study with a focus on the lack of access to needed resources. Financial support for more professional interpretive resources is needed as the lack of such resources was expressed as a concern in this study. Language support services ought to be within federal, provincial and municipal plans to ensure health equity and patient safety when a language barrier exists. There needs to be a language of inclusivity and equity in political spaces as what is discussed and deemed important in such spaces permeates down to organizations, agencies, populations, communities, families, and individuals.

All nurses, in this present study, voiced the importance of language within the healthcare space as critical to ensuring patient safety and person-centered care. Results of this research can inform policy makers of NRT job structure and organizational supports or lack thereof to ensure that nurses are provided with the supports, resources, and services to ensure culturally safe delivery of nursing care.

\section{Practice}

Developing inclusive practice environments. Practice environments ought to provide a culture of guidance and support to ensure patient safety is maintained when language barriers 
exist. In this present study, the majority of the nurses did not receive formal organizational orientation about available interpreter resources, which would have been helpful in first establishing awareness of the resources. The availability of and being aware of resources is a necessary prerequisite for the use of resources by healthcare staff, specifically nurses as noted in this present study and reflected in the literature (Brisset et al., 2014). Supports and resources were either not available or the nurses were not aware of these supports and resources which served as a challenge in facilitating care encounters when a language barrier was present. The location of interpreter tools within healthcare organizations needs to be considered and improved, as nurses in this present study found that the current placement of resources was not conducive to their nursing work environment and workload. Nurses wanted immediate access to resources, in other words, for resources to be in close reach (e.g. more portable mobile call-in interpretative phones carried by nurses and call-in interpreter services to be at the bedside).

Continued professional development. Nurses, in this present study, shared the challenges they had with remembering where to access the resources or how to use the resources after their initial orientation. The lack of ongoing professional development and training was cited as a reason for not using resources. To ensure HCPs are maintaining their knowledge of how and where to access the organizational resources, knowledge of interpretation tools in healthcare spaces (Brisset et al., 2014), continued professional development and training (Brisset et al., 2014), and ongoing in-services and workshops (Hendson et al., 2015) are important considerations.

Incorporating equitable practices into workload measures. In this present study, the need for additional time to provide appropriate, culturally safe care was considered important. For care to be equitable for patients that do not share the same language as the nurse, the nurses 
suggested that there be more consideration for the workloads of nurses and more time allotted when caring for patients when language barriers exist. Therefore, with patient assignments, it is important that sufficient time is allotted to ensure nursing care can be tailored to individual patient needs resulting in quality and safe nursing practice. Thus, as the NRT nurses voiced concerns with not being provided with additional time to care for a patient when there was a language barrier, there needs to be a consideration of the time it takes to span the relational distance between the nurse and patient in order to achieve a shared meaning.

\section{Further research}

It is important to understand the impact that language barriers have on the provision of quality and safe care. Therefore, a thorough organizational assessment of the current inventory of services would be essential in examining areas for improvement. This assessment should include all hospital units, as nurses in this study found that different units had different supports and resources available. This will also contribute to a further understanding of the various types of nursing care provided and what language service needs are required.

It would also be important to include the voices of the nurses working in these various units as they are the ones needing to access and use these supports and resources. Moreover, this study recruited nurses within or near an urban city and thus further exploration of experiences of nurses working in hospitals in rural settings would bring forth another dimension to the literature.

Further research on NRTs is needed to explore the existing tools nurses use within the context of a language barrier to assess the tools' efficacy. A better understanding of administrative demands regarding budgetary constraints would also be key to a better 
understanding of the NRT nurses' experiences when caring for patients when a language barrier exists.

During data analysis, I was attentive to the fact that I did not collect data on the patient perspective. Therefore, research from the patient perspective regarding the effectiveness of interpretive resources within the nurse-patient relationship is important.

In this study, due to feasibility, a single interview instead of multiple interviews was conducted. However incorporating more than one interview could potentially elicit further enriched experiential accounts from the NRT nurses.

Finally, conducting a quantitative research study using a survey or questionnaire developed from the findings of this study would allow for a larger number of NRT nurses, from several hospital sites and locales, to participate perhaps leading to an even deeper understanding of their experiences.

\section{Lessons Learned from the Field}

Although several implications arose from this study, there are lessons learned from the field. The target population of this study were nurses, where understanding "language barrier" was explored from one side of the supposed barrier. I did not collect data on the patient perspective but was mindful of this throughout the research process and in particular, during data analysis by engaging in critical reflection. In addition, I recruited nurses within or near an urban city and thus the insights of nurses working in differing locales such as rural areas were not explored. Lastly, achieving resonance was achieved through the selection of in-vivo quotes depicting experiential accounts of the nurses' experiences. The choice of quotes was completed through a comprehensive analysis by me and by my thesis committee members. However, due to the time constraints of this student-led research study, going back to the participants to ensure the 
findings were reflective of their experience was not done and could have potentially enriched the data analysis.

\section{Study Summary}

Interpretive phenomenology explores the meaning of the phenomenon of interest through interpretive inquiry (Tuohy et al., 2013) and thus exploration of the meaning of language barriers for these NRT nurses was of particular interest. The four existentials fundamental to human existence (van Manen, 1997) provided a deeper understanding of the meaning of the nurses' experiences. van Manen's (1997) thematic analysis was used to analyze the interviews. From the data analysis, four themes emerged encompassing the experiences of NRT nurses caring for patients when a language barrier exists. These four themes were (a) Trying to span the distance,

(b) Everything comes down to time, (c) Desiring shared meaning, and (d) Experiencing disequilibrium.

Through interpretive phenomenological analysis, a deepened and enriched understanding of the NRT nurses' experiences was attained leading to a better understanding of how these nurses experience the impact of systemic barriers that shape the provision of care with patients where a language barrier exists. The nurses tried to span the relational distance between themselves and patients by playing guessing games, finding and using resources and by just muddling through. However, the nurses found that everything came down to time which caused a lack of timely care, caused the nurses to feel the pressure of time which was exacerbated by needing to take the time to translate. Nonetheless, the nurses desired a shared meaning between themselves and patients and achieved this by building emotional connections, expressing compassion and empathy, and resorting to reductive relations. However, in caring for patients in 
the context of a language barrier, the nurses experienced disequilibrium or imbalance as they felt frustration while also embodying skills that facilitated their care encounters.

Nurses are in a position to advocate for systemic change. The provision of their care becomes sub-optimal care as the basis for good care is relational care, which becomes undermined in the context of language barriers as evident by the experiences of nurses in this present study. Collective action from organizations, researchers, HCPs and members of political spaces is also important in ensuring inclusivity in healthcare. This deeper understanding of the nurses' experiences resulted in several implications for policy, practice, and further research. van Manen's (2014) thoughts on phenomenology were included to introduce this present study and are repeated here as a reminder that it will be up to the readers of this thesis to attain their own meaning: "Nothing is more meaningful than the quest for meaning, the mystery of meaning, how meaning originates and occurs --- as well as the meaning of our responsibility for others and the organic, material, and technological world we inhabit" (p.13). 


\section{Appendices}

\section{Appendix A: Letter Requesting Approval to Recruit \& Approval Email}

Dr. Nancy Walton,

Director and Associate Professor,

Daphne Cockwell School of Nursing

Ryerson University

350 Victoria St,

Toronto, ON, M5B 2K3

Dear Dr. Nancy Walton,

Hope all is well. My name is Niyat Mehari and I am a MN student. I am writing this to obtain approval in recruiting potential participants for a Master's of Nursing thesis entilited: Language Discordance: Perspectives from Resource Team Nurses. The purpose of this interpretive phenomenological study is to explore and understand the meaning of resource team nurses' lived experiences of language discordance from an academic institution in an urban city, in Canada. The recruitment of 5-25 nurses through the Master of Nursing and Post-Diploma program will be sought for a 60 -minute one individual interview. Participant selection will be based on the following: participants having experienced the phenomenon of interest, are currently practicing in a nursing resource team, and are able to communicate their experience(s) with the language discordance.

Participant recruitment will be sought through three avenues. First, an email will be sent to the registered nursing students enrolled in the Master of Nursing (MN) and Post-Diploma programs, with a brief description of the study. Second, recruitment will be sought through posting the recruitment handout on the nursing information board in the nursing floor (POD Floor 4) and graduate lounge. Lastly, a 5-minute speech at the beginning of MN and PostDiploma classes being taught during the Winter and Spring 2018 term.

I anticipate to submit the proposal of the study to REB upon approval. REB requires an approval letter conveying approval from the Director of the Daphne Cockwell School of Nursing at Ryerson University.

Sincerley,

Niyat Mehari

$\mathrm{RN}, \mathrm{BScN}, \mathrm{MN}$ student 


\section{Approval Email Granted}

- Nancy Walton

Feb 8 (2 days ago)

to Sepali, me -

Dear Niyat,

Thank you for getting in touch.

I wanted to connect with the Graduate Program Director, Dr. Suzanne Fredericks and the Associate Director of our Post Diploma Degree Program to confirm that they are both in agreement with you recruiting from the programs.

They've both agreed it is absolutely fine.

The only note l'Il make is that you'll need to check in with them and individual professors re recruiting in class, even just using a short time at the outset of class. There may be some individual professors who would not support this, so that method might be not as straightforward as your other proposed methods. Just a note for you to consider. Otherwise, we are in agreement that you may recruit from the students for your project.

This email should suffice for the REB application. Please feel free to append it to your application. Let me know if we can assist in any other way and best of luck with your research.

Best,

Nancv 


\section{Appendix B: REB Approval}

\section{Research Ryerson Ethics Board University}

To: Niyat Mehari

Nursing

Re: REB 2018-070: Language Discordance: Prespectives from Resource Team Nurses

Date: March 22, 2018

Dear Niyat Mehari,

The review of your protocol REB File REB 2018-070 is now complete. The project has been approved for a one year period. Please note that before proceeding with your project, compliance with other required University approvals/certifications, institutional requirements, or governmental authorizations may be required.

This approval may be extended after one year upon request. Please be advised that if the project is not renewed, approval will expire and no more research involving humans may take place. If this is a funded project, access to research funds may also be affected.

Please note that REB approval policies require that you adhere strictly to the protocol as last reviewed by the REB and that any modifications must be approved by the Board before they can be implemented. Adverse or unexpected events must be reported to the REB as soon as possible with an indication from the Principal Investigator as to how, in the view of the Principal Investigator, these events affect the continuation of the protocol.

Finally, if research subjects are in the care of a health facility, at a school, or other institution or community organization, it is the responsibility of the Principal Investigator to ensure that the ethical guidelines and approvals of those facilities or institutions are obtained and filed with the REB prior to the initiation of any research.

Please quote your REB file number (REB 2018-070) on future correspondence.

Congratulations and best of luck in conducting your research.

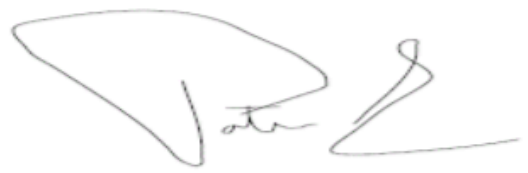

Dr. Patrizia Albanese, $\mathrm{PhD}$

Chair, Ryerson University Research Ethics Board

The Following protocol attachments have been reviewed and approved. 


\section{Appendix C: Recruitment- Professor Email}

Dear Professor,

I am currently conducting a thesis in the Master of Nursing Program entitled, "Language Discordance: Perspectives from Resource Team Nurses”. The purpose of this study is to explore and understand the meaning of resource team nurses' lived experiences of language discordance. Little is known about the phenomenon and there is limited literature from the voices of resource team nurses.

One of my recruiting methods is through a 5-minute speech in the beginning of $\mathrm{MN}$ and Postdiploma classes. I am interested in presenting in your next class, or another date if best. Please don't hesitate to contact for questions you may have.

This research study has been reviewed and approved by the Ryerson University Research Ethics Board.

Sincerley,

Niyat Mehari

$\mathrm{RN}, \mathrm{BScN}, \mathrm{MN}$ student 


\section{Appendix D: REB Amendment Approval}

Ryerson

University

Ryerson Home > Online Ethics Submission and Review System > Protocol View

\section{Email sent to applicant on May 25, 2018 11:55:10 AM}

From: rebchair@ryerson.ca

To: miyad1.mehari@ryerson.ca, sguruge@ryerson.ca

Cc: rebchair@ryerson.ca

Subject: REB 2018-070 Status

Date: May 25, 2018 11:55:10 AM

REB 2018-070

Project Title: Language Discordance: Perspectives from Resource Team Nurses

Dear Niyat Mehari,

Thank you very much for the submission of amendments for the above project. The Research Ethics Board has completed the review of your resubmission and the proposed amendments have been approved. This does not change the approval status nor the original approval date of the project.

Congratulations and best of luck with the project.

Please quote your REB file number (2018-070) on future correspondence.

If you have any questions regarding your submission or the review process, please do not hesitate to get in touch with the Research Ethics Board (contact information below).

No research involving humans shall begin without the prior approval of the Research Ethics Board.

This is part of the permanent record respecting or associated with a research ethics application submitted to Ryerson University.

NOTE: This email account (rebchair@ryerson.ca) is monitored by multiple individuals. If you wish to contact a specific member of the Research Ethics Board, please do so directly.

Yours sincerely,

Zakiya Atcha, MSW

Research Ethics Co-Ordinator

on behalf of:

Dr. Patrizia Albanese, PhD

Chair, Ryerson University Research Ethics Board

(416) $979-500 n$ ext 6526 


\section{Appendix E: Recruitment- Email/Handout/Speech for Potential Participants}

\section{Seeking Resource Team Nurses to Participate in a Research Study}

\section{Email/ in-person script}

$\mathrm{Hi}$,

My name is Niyat Mehari. I am a Master of Nursing student at Ryerson University in the Daphne Cockwell School of Nursing I am contacting you to see if you might be interested in participating in a research study.

This research is being done as part of my Masters project and my supervisor's name is Dr. Sepali Guruge. The focus of the research is exploring and understanding the meaning of resource team nurses' lived experiences of language discordance from an academic institution in an urban city, in Canada.

To participate you need to: currently be working as part of a resource team (i.e. a resource team (float pool) nurse); experienced language discordance (language barrier) in practice and be able to communicate your experience (s) with language discordance?

If you agree to volunteer, you will be asked to talk about your experience in an interview.

Your participation will involve one approximately 60 -90 minute semi-structured interview. This will be the maximum time expected for the interview.

In appreciation of your time, travel, childcare costs, you will receive $\$ 20$.

Your participation is completely voluntary and if you choose not to participate it will not impact the relationship with the researcher, Niyat Mehari or your relationship with Ryerson University.

The research has been reviewed and approved by the Ryerson University Research Ethics Board.

If you are interested in more information about the study or would like to volunteer please contact Niyat Mehari, RN, BScN, MN student: miyad1.mehari@,ryerson.ca.

If you know other nurses that may be interested, please forward this to them. 
RYERSON

UNIVERSITY

\section{PARTICIPANTS NEEDED FOR RESEARCH IN NURSING}

Language Discordance: Perspectives from Resource Team Nurses

Are You/Have You:

- Currently working as part of a resource team (i.e. a resource team (float pool) nurse)?

- Experienced language discordance (language barrier) in practice?

- Able to communicate your experience (s) with language discordance?

If you answered yes to the above noted questions you are invited to volunteer in this study of exploring and understanding the meaning of resource team nurses' lived experiences of language discordance from an academic institution in an urban

$$
\text { city, in Canada. }
$$

You will be asked to talk about your experience in an interview.

Your participation will involve one approximate 60-90 minute semi-structured interview. This will be the maximum time expected for the interview.

In appreciation of your time, travel, transportation and child care costs, you will receive \$20.

If you are interested in participating in this study or for more information, please contact:

Niyat Mehari (researcher/MN student)

Email: miyad1.mehari@ryerson.ca

This study will contribute to a Master of Nursing thesis.

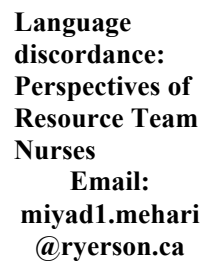

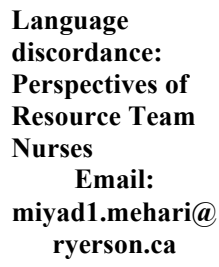

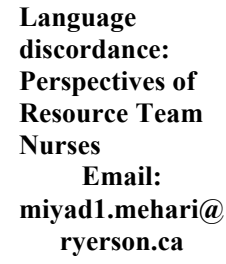

Language discordance: Perspectives of Resource Team Nurses Email: miyad1.mehari@ ryerson.ca

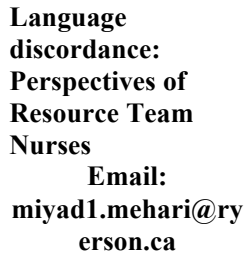

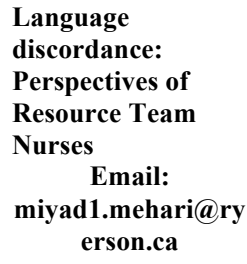

Language
discordance:
Perspectives of
Resource Team
Nurses
Email:
miyad1.mehari@ry
erson.ca 


\section{Appendix G: Consent Form}

Ryerson

Daphne Cockwell

School of Nursing

Faculty of Community Services

\section{Ryerson University \\ Consent Agreement}

\section{Language Discordance: Perspectives from Resource Team Nurses}

You are being invited to participate in a research study, that will contribute to a Master of Nursing thesis. Please read this consent form so that you understand what your participation will involve. Before you consent to participate, please ask any questions to be sure you understand what your participation will include.

\section{Investigators and Thesis Committee:}

This research study is being conducted by Niyat Mehari (RN, BScN, MN student).

Thesis Supervisor: Dr. Sepali Guruge (RN, BScN, MSc, PhD), from the Daphne Cockwell School of Nursing at Ryerson University.

Thesis Committee: Dr. Susan E. Bishop (RN, BScN, MSc, PhD) \& Dr. Oona St-Amant (RN, $\mathrm{PhD})$.

\section{Purpose of the Study:}

This study will seek the meaning of language discordance of 5-10 nurses who are currently working on a nursing resource team. The purpose of this study is to explore and understand the meaning of resource team nurses' lived experiences of language discordance from an academic institution in an urban city, in Canada. According to the literature, language discordance is defined as the barrier to effective communication between the patient and health care practitioner due to a lack of proficiency with the same language(s).

\section{Description of the Study:}

By exploring the meaning of language discordance for registered nurses of the resource team, I hope to highlight the significance of language within interactions of health professionals and patients. Also, I hope to explore the meaning of language discordance through their experiences in navigating through several inpatient units.

If you consent to participate in this study, you will be asked to take part in one approximately 6090 minute face-to-face interview. The interviews will be audio recorded using a digital recorder so the interview can be typed later. You will be interviewed at a private/secure location on campus, such as a private room that can be booked on the Nursing floor, as this will provide aural and visual privacy in sharing your experience(s),

The questions you will be asked will encompass your experiences as a resource team nurse with language discordance. Your insight is important in gaining an understanding of language discordance through the perspective of resource team nurses. If you would like to obtain a copy 
of the research findings, please contact the researcher (Niyat Mehari) by end of 2018, and you will receive an emailed copy of study results.

\section{Confidentiality:}

You will be known to the researcher and thus a commitment to confidentiality will be maintained. Audio recordings from the interviews will be deleted after transcription. Data will be transcribed by the researcher. You will be assigned a unique code, to be used to identify you throughout the research process so that no names or identifiers will be utilized in the transcripts or in the thesis. The original consent forms and interview audio-recordings will only be accessed by the researcher. Researcher, supervisor and thesis committee will be reviewing the study data transcripts with the assigned unique code to each transcript. Data will not be reported in a way that would disclose the identity of the participants. Furthermore, identifiable information will not be used in any publications. Transcripts and coding lists will be password protected. Consent forms will be kept in a locked cabinet in thesis supervisor's office at Ryerson University. Your workplace will be unaware of their participation in your study. Documents to be kept for five years, if requiring secondary analysis, and will then be destroyed.

\section{Potential Benefits of the Study:}

A potential benefit of participating is that you will have the opportunity to share your insights and experiences as a nursing resource team nurse. There is no guarantee, however, that you will receive any direct benefits from participating in this study.

\section{Potential Risks or Discomforts:}

Questions will be asked as you share your experience (s) with language discordance as a nursing resource team nurse. There is a possibility of discomfort, in that some of the questions may make you feel uncomfortable. You may skip to the next question or stop participation, either briefly or permanently. You do not need to provide a reason for skipping questions, stopping the interview, or withdrawing from the study.

In addition, the researcher is a current student, that you may or may not have a pre-existing relationship with prior to this study. This consent form provides a full disclosure of all the information necessary for you to make an informed decision about participating in this study. If you volunteer to participate, it is completely voluntary.

\section{Incentives to Participation:}

Participation is completely voluntary. If you wish to volunteer, you will receive $\$ 20$ in consideration of your time, travel, transportation, and child care costs. Participants will not be paid to participate in this study.

\section{Voluntary Participation and Withdrawal:}

Participation in this study is completely voluntary. You can choose whether to be in this study or not. If any question makes you uncomfortable, you can skip that question. You may stop participating at any time. Any data gathered prior to a notification of withdrawal will be destroyed immediately upon confirmation of your request to withdraw. Due to the nature of limited time of this study, withdrawal from this study is not possible after 2-weeks of the interview completion, and thus withdrawal, can occur anytime prior to this 2-week window. Your choice of whether or not to participate will not influence your future relations with Ryerson University or the researcher, Niyat Mehari involved in the research. 


\title{
Questions about the Study:
}

If you have any questions or concerns about the research at anytime, please feel free to contact

\author{
Niyat Mehari (researcher/MN student) \\ miyad1.mehari@ryerson.ca \\ Or \\ Dr. Sepali Guruge (Thesis Supervisor) \\ sguruge@ryerson.ca
}

If you have questions regarding your rights as a human subject and participant in this study, you may contact the Ryerson University Research Ethics Board for information.

\author{
Research Ethics Board \\ c/o Office of the Vice President, Research and Innovation \\ Ryerson University \\ 350 Victoria Street \\ Toronto, ON M5B 2K3 \\ 416-979-5042
}




\section{Language Discordance: Perspectives from Resource Team Nurses}

\section{Confirmation of Agreement:}

Your signature below indicates that you have read the information in this agreement and have had a chance to ask any questions you have about the study. Your signature also indicates that you agree to participate in the study and have been told that you can change your mind and withdraw your consent to participate at any time. You have been given a copy of this agreement.

You have been told that by signing this consent agreement you are not giving up any of your legal rights.

Name of Participant (please print)

Signature of Participant

Date

I agree to be audio-recorded for the purposes of this study. I understand how these recordings will be stored and destroyed.

Date 


\section{Appendix H: Interview Guide}

\section{Role Specific and Demographic Questions}

How long have you been working as a nurse (Ian, Nakamura-Florez, \& Lee, 2016)?

How long have been working as a nursing resource team nurse?

Tell me about you and your role? What type of units do you work in?

What other languages do speak other than English or French?

\section{Opening Question}

"As you already know, I am conducting this study to learn more about the lived experiences of resource team nurses with language discordance. What has been your experience with language discordance or language barriers in practice?"

\section{Potential Follow-up Questions}

What experiences have you had with language discordance in providing care?

- What is like to communicate when there is a discordance in language?

- How have these experiences influenced your nursing practice (Ian, Nakamura-Florez, $\&$ Lee, 2016)

What is the significance of language within interactions of health professionals and patients?

What meaning do you give you this phenomenon within the context of the healthcare environment?

\section{Clarifying Questions and Probes}

"Can you think of a specific example, situation, event or person?" (van Manen, 1997)

"In that experience, how did you feel?" "What did it feel like?"

"Can you explain further about that experience? What was it like?

"Can you describe what that means to you?" 


\section{References}

Ahmad, F., Mahmood, S., Pietkiewicz, I., McDonald, L., \& Ginsburg, O. (2012). Concept mapping with south Asian immigrant women: Barriers to mammography and solutions. Journal of Immigrant Minority Health, 14(2), 242-250. doi: 10.1007/s10903-011-9472-7

Anderson, J., Perry, J., Blue, C., Browne, A., Henderson, A., Khan, K. B., . . Smye, V. (2003). "Rewriting" cultural safety within the postcolonial and postnational feminist project: Toward new epistemologies of healing. Advances in Nursing Science, 26(3), 196-214. doi:10.1097/00012272-200307000-00005

Anderson, L. M., Scrimshaw, S. C., Fullilove, M. T., Fielding, J. E., \& Normand, J. (2003). Culturally competent healthcare systems. American Journal of Preventive Medicine, 24(3), 68-79. doi:10.1016/S0749-3797(02)00657-8

Árnason, V. (2000). Gadamerian dialogue in the patient-professional interaction. Medicine, Health Care and Philosophy,_3(1), 17-23. doi:10.1023/A:1009908132170

Asanin, J., \& Wilson, K. (2008). “I spent nine years looking for a doctor”: Exploring access to health care among immigrants in Mississauga, Ontario, Canada. Social Science \& Medicine (1982), 66 (6), 1271-1283. doi:10.1016/j.socscimed.2007.11.043

Berger, R. (2015). Now I see it, now I don't: Researcher's position and reflexivity in qualitative research. Qualitative Research, 15(2), 219-234. doi:10.1177/1468794112468475

Binder, P., Borné, Y., Johnsdotter, S., \& Essén, B. (2012). Shared language is essential: Communication in a multiethnic obstetric care setting. Journal of Health Communication, 17(10), 1171-1186. doi:10.1080/10810730.2012.665421

Bischoff, A., Bovier, P. A., Isah, R., Francoise, G., Ariel, E., \& Louis, L. (2003). Language 
barriers between nurses and asylum seekers: Their impact on symptom reporting and referral. Social Science and Medicine, 57(3), 503-512. doi:10.1016/S0277-9536(02)003763

Bischoff, A., \& Denhaerynck, K. (2010). What do language barriers cost? an exploratory study among asylum seekers in switzerland. BMC Health Services Research, 10(1), 248-248. doi:10.1186/1472-6963-10-248

Blackford, J., \& Street, A. (2002). Cultural conflict: The impact of western feminism(s) on nurses caring for women of non-english speaking background. Journal of Clinical Nursing, 11(5), 664-671. doi:10.1046/j.1365-2702.2002.00680.x

Bridges, J., Nicholson, C., Maben, J., Pope, C., Flatley, M., Wilkinson, C., Meyer, J., \& Tziggili, M. (2013). Capacity for care: meta-ethnography of acute care nurses' experiences of the nurse-patient relationship. Journal of Advanced Nursing, 69(4), 760-772. doi: $10.1111 /$ jan. 12050

Brisset, C., Leanza, Y., Rosenberg, E., Vissandjée, B., Kirmayer, L., Muckle, G., . . Laforce, H. (2014). Language barriers in mental health care: A survey of primary care practitioners. Journal of Immigrant and Minority Health, 16(6), 1238-1246. doi:10.1007/s10903-013-9971-9

Caldas, C. P., \& Berterö, C., (2012). A concept analysis about temporality and its applicability in nursing care. Nursing Forum, 47(4), 245-252. doi:10.1111/j.1744-6198.2012.00277.x

Campinha-Bacote, J. (1999). A model and instrument for addressing cultural competence in health care. Journal of Nursing Education, 38(5), 203-7. Retrieved from 
http://ezproxy.lib.ryerson.ca/login?url=https://search-proquest-

com.ezproxy.lib.ryerson.ca/docview/203962985?accountid=13631

Chan, Y., Alagappan, K., Rella, J., Bentley, S., Soto-Greene, M., \& Martin, M. (2010).

Interpreter services in emergency medicine. Journal of Emergency Medicine, 38(2), 133-

139. doi:10.1016/j.jemermed.2007.09.045

Chen, Y. Y. B., Li, A. T., Fung, K. P-L., \& Wong, J. P-H., (2015). Improving access to mental health services for racialized immigrants, refugees, and non- status people living with HIV/AIDS. Journal of Health Care for the Poor and Underserved, 26(2), 505-518.

Retrieved from http://ezproxy.lib.ryerson.ca/login?url=https://search-proquestcom.ezproxy.lib.ryerson.ca/docview/1679411500?accountid=13631

Coleman, J., \& Angosta, A. D. (2017). The lived experiences of acute-care bedside registered nurses caring for patients and their families with limited English proficiency: A silent shift. Journal of Clinical Nursing, 26(5-6), 678-689. doi:10.1111/jocn.13567

Creswell, J. W. (2013). Qualitative inquiry and research design: Choosing among five approaches $\left(3^{\text {rd }}\right.$ ed.). Thousand Oaks, CA: Sage Publications.

de Witt, L., \& Ploeg, J. (2006). Critical appraisal of rigour in interpretive phenomenological nursing research. Journal of Advanced Nursing, 55(2), 215-229. doi:10.1111/j.1365 2648.2006.03898.x

Diamond, L.C., \& Jacobs, E. A. (2010). Let's not contribute to disparities: The best methods for teaching clinicians how to overcome language barriers to health care. Journal of General Internal Medicine, 25 (Suppl 2), 189-193. doi:10.1007/s11606-009-1201-8 
Diamond, L. C., Schenker, Y., Curry, L., Bradley, E. H., \& Fernandez, A. (2009). Getting by: Underuse of interpreters by resident physicians. Journal of General Internal Medicine, 24(2), 256-262. doi:10.1007/s11606-008-0875-7

Dowling, M. (2007). From Husserl to van Manen. A review of different phenomenological approaches. International Journal of Nursing Studies, 44(1), 131-142. doi:10.1016/j.ijnurstu.2005.11.026

Dziuba-Ellis, J. (2006). Float pools and resource teams: A review of the literature. Journal of Nursing Care Quality, 21(4), 352-359. doi:10.1097/00001786-200610000-00013

Edwards, R., Temple, B., \& Alexander, C. (2005). Users' experiences of interpreters: The critical role of trust. Interpreting, 7(1), 77-95. doi:10.1075/intp.7.1.05edw

Eiland, H. (1984). The way to nearness: Heidegger's interpretation of presence. Philosophy and Literature, 8(1), 43-54. doi:10.1353/phl.1984.0039

Enarson, D. A., \& Ait-Khaled, N. (1999). Cultural barriers to asthma management. Pediatric Pulmonology, 28(4), 297-300. doi:10.1002/(SICI)1099-0496(199910)28:4<297::AIDPPUL9>3.0.CO;2-S

Eneriz-Wiemer, M., Sanders, L.M., Barr, D.A., \& Mendoza, F. S. (2014). Parental limited English proficiency and health outcomes for children with special health care needs: a systematic review. Academic Pediatrics, 14(2), 128-136. doi:10.1016/j.acap.2013.10.003

Feo, R., Rasmussen, P., Wiechula, R., Conroy, T., \& Kitson, A. (2017). Developing effective and caring nurse-patient relationships. Nursing Standard (Royal College of Nursing (Great Britain):1987), 31(28), 54-63. doi:10.7748/ns.2017.e10735 
Flood, A. (2010). Understanding phenomenology. Nurse Researcher (through 2013), 17(2), 715. Retrieved from http://ezproxy.lib.ryerson.ca/login?url=https://search-proquestcom.ezproxy.lib.ryerson.ca/docview/200780602?accountid=13631

Garneau, A. B., \& Pepin, J. (2015). Cultural competence: A constructivist definition. Journal of Transcultural Nursing, 26(1), 9-15. doi:10.1177/1043659614541294

Gray, D. P., \& Thomas, D. J. (2006). Critical reflections on culture in nursing. Journal of Cultural Diversity, 13(2), 76-82. Retrieved from http://ezproxy.lib.ryerson.ca/login?url=https://search-proquestcom.ezproxy.lib.ryerson.ca/docview/219375175?accountid=13631

Gillon, R. (1994). Medical ethics: Four principles plus attention to scope. BMJ: British Medical Journal, 309(6948), 184-188. doi:10.2307/29724194

Giorgi, A. (2005). The phenomenological movement and research in the human sciences. Nursing Science Quarterly, 18(1), 75-82. doi:10.1177/0894318404272112

Hadziabdic, E., Albin, B., Heikkilä, K., \& Hjelm, K. (2014). Family members' experiences of the use of interpreters in healthcare. Primary Health Care Research \& Development, 15(2), 156-169. doi:10.1017/S1463423612000680

Halldorsdottir, S. (2008). The dynamics of the nurse-patient relationship: Introduction of a synthesized theory from the patient's perspective. Scandinavian Journal of Caring Sciences, 22(4), 643-652. doi:10.1111/j.1471-6712.2007.00568.x

Hampers, L. C., \& McNulty, J. E. (2002). Professional interpreters and bilingual physicians in a pediatric emergency department: Effect on resource utilization. Archives of Pediatrics \& Adolescent Medicine, 156(11), 1108-1113. doi:10.1001/archpedi.156.11.1108 
Hawley, M., \& Jensen, L. (2007). Making a difference in critical care nursing practice. Qualitative Health Research, 17(5), 663-674. doi:10.1177/1049732307301235

Health Quality Ontario. (n.d.). What is health quality - Health equity and quality. Retrieved from http://www.hqontario.ca/What-is-Health-Quality/Health-Equity-and-Quality

Hemann, M., \& Davidson, G. (2012). Perspective of a float pool model in ambulatory care. Medsurg Nursing: Official Journal of the Academy of Medical-Surgical Nurses, 21(3), 164-6, 170. Retrieved from http://ezproxy.lib.ryerson.ca/login?url=https://search.proquest.com/docview/1021184801?a ccountid $=13631$

Hendson, L., Reis, M. D., \& Nicholas, D. B. (2015). Health care providers' perspectives of providing culturally competent care in the NICU. Journal of Obstetric, Gynecologic, \& Neonatal Nursing, 44(1), 17-27. doi:10.1111/1552-6909.12524

Higginbottom, G. A., Safipour, J., Yohani, S., O’Brien, B., Mumtaz, Z., \& Paton, P. (2015). An ethnographic of communication challenges in maternity care for immigrant women in rural Alberta. Midwifery, 31(2), 297-304. doi: 10.1016/j.midw.2014.09.009

Ian, C., Nakamura-Florez, E., \& Lee, Y. (2016). Registered nurses' experiences with caring for non-English speaking patients. Applied Nursing Research, 30(Complete), 257-260. doi:10.1016/j.apnr.2015.11.009

Jacobs, E. A., Shepard, D. S., Suaya, J. A., \& Stone, E. (2004). Overcoming language barriers in health care: Costs and benefits of interpreter services. American Journal of Public Health, 94(5), 866-869. doi:10.2105/AJPH.94.5.866 
Jacobs, E., Chen, A. H., Karliner, L. S., Agger-Gupta, N., \& Mutha, S. (2006). The need for more research on language barriers in health care: A proposed research agenda. The Milbank Quarterly, 84 (1), 111-133. doi: 10.1111/j.1468-0009.2006.00440.x

Jacobs, E. A., Diamond, L. C., \& Stevak, L. (2010). The importance of teaching clinicians when and how to work with interpreters. Patient Education and Counselling, 78(2), 149-153. doi:10.1016/j.pec.2009.12.001

John-Baptiste, A., Naglie, G., Tomlinson, G., Alibhai, S. M. H., Etchells, E., Cheung, A., . . Krahn, M. (2004). The effect of English language proficiency on length of stay and inhospital mortality. Journal of General Internal Medicine, 19(3), 221-228. doi:10.1111/j.1525-1497.2004.21205.x

Jones, T. L. (2010). A holistic framework for nursing time: Implications for theory, practice, and research. Nursing Forum, 45(3), 185. doi:10.1111/j.1744-6198.2010.00180.x

Jootun, D. (2009). Reflexivity: promoting rigour in qualitative research. Nursing Standard, 23 (23), 42-46. doi:10.7748/ns2009.02.23.23.42.c6800

Kale, E., \& Syed, H. R. (2010). Language barriers and the use of interpreters in the public health services. A questionnaire-based survey. Patient Education and Counseling, 81(2), 187191. doi:10.1016/j.pec.2010.05.002

Kalich, A., Heinemann, L., \& Ghahari, S. (2016). A scoping review of immigrant experience of health care access barriers in Canada. Journal of Immigrant and Minority Health, 18(3), 697-709. doi:10.1007/s10903-015-0237-6

Karliner, L. S., Jacobs, E. A., Chen, A. H., \& Mutha, S. (2007). Do professional interpreters improve clinical care for patients with limited English proficiency? A systematic review of 
the literature. Health Services Research, 42(2), 727-754. doi: 10.1111/j.14756773.2006.00629.x

Kirkham, S. R., \& Browne, A. J. (2006). Toward a critical theoretical interpretation of social justice discourses in nursing. ANS. Advances in Nursing Science, 29(4), 324-339. doi:10.1097/00012272-200610000-00006

Kirmayer, L. (2012). Rethinking cultural competence. Transcultural Psychiatry, 49(2). doi: $10.1177 / 1363461512444673$

Kitson, A. (1993). Formalizing concepts related to nursing and caring. In Kitson, A. (Ed.), Nursing: Art and Science (25-47). London: Chapman \& Hall.

Laher, N., Sultana, A., Aery, A., \& Kumar, N. (2018). Access to language interpretation services and its impact on clinical and patient outcomes: A scoping review. Retrieved from http://www.wellesleyinstitute.com/wp-content/uploads/2018/04/Language-InterpretationServices-Scoping-Review.pdf

Langdridge, D. (2007). Phenomenological psychology: theory, research and method. Harlow: Pearson Education Ltd.

Larson, N., Sendelbach, S., Missal, B., Fliss, J., \& Gaillard, P. (2012). Staffing patterns of scheduled unit staff nurses vs. float pool nurses: A pilot study. Medsurg Nursing: Official Journal of the Academy of Medical-Surgical Nurses, 21(1), 27-32, 39. Retrieved from, http://ezproxy.lib.ryerson.ca/login?url=http://search.proquest.com.ezproxy.lib.ryerson.ca/d ocview/922981830?accountid=13631

Law, M., Wilson, K., Eyles, J., Elliott, S., Jerrett, M., Moffat, T., \& Luginaah, I. (2005). Meeting health need, accessing health care: The role of neighbourhood. Health \& Place, 11(4), 367-377. doi:10.1016/j.healthplace.2004.05.004 
Leanza, Y., Miklavcic, A., Boivin, I., \& Rosenberg E. (2014). Working with interpreters. In: Kirmayer, L., Rousseau, C., \& Guzder, J., editors. Cultural consultation: encountering the other in mental health care. New York: Springer.

Lebanik, L. \& Britt, S. (2015). Float pool nurses come to the rescue. Nursing, 45 (3). doi: 10.1097/01.NURSE.0000460715.73128.ea

Lee, H.Y., \& Vang, S. (2010). Barriers to cancer screening in Hmong Americans: the influence of health care accessibility, culture, and cancer literacy. Journal of Community Health, 35(3), 302-314. doi:10.1007/s10900-010-9228-7

Linzer, P., Tilley, A. M., \& Williamson, M. V. (2011). What floats a float nurse's boat? Creative Nursing, 17(3), 130-8. Retrieved from http://ezproxy.lib.ryerson.ca/login?url=https://search-proquestcom.ezproxy.lib.ryerson.ca/docview/884337633?accountid=13631

Lofters, A.K., Moineddin, R., Hwang, S.W., \& Glazier, R. H. (2011). Predictors of low cervical cancer screening among immigrant women in Ontario, Canada. BMC Women's Health, 11 (1), 1-11. doi: 10.1186/1472-6874-11-20

Lor, M., Xiong, P., Schwei, R. J., Bowers, B.J., Jacobs, E. A., (2016). Limited English proficient Hmong- and Spanish-speaking patients' perceptions of the quality of interpreter services. Journal of Nursing Studies, 54 (Complete), 75-83. doi: https://doi.org/10.1016/j.ijnurstu.2015.03.019

Mackey, S. (2005). Phenomenological nursing research: Methodological insights derived from Heidegger's interpretive phenomenology. International Journal of Nursing Studies, 42(2), 179-186. doi:10.1016/j.jinurstu.2004.06.011 
Maggs-Rapport, F. (2001). 'Best research practice': In pursuit of methodological rigour. Journal of Advanced Nursing, 35(3), 373-383. doi:10.1046/j.1365-2648.2001.01853.x

Matua, G. A. (2015). Choosing phenomenology as a guiding philosophy for nursing research. Nurse Researcher, 22(4), 30-34. doi: 10.7748/nr.22.4.30.e1325

Matwick, A. L., \& Woodgate, R. L. (2017). Social justice: A concept analysis. Public Health Nursing, 34(2), 176-184. doi:10.1111/phn.12288

McEwen, M. \& Wills, E. M. (2014). Theoretical basis for nursing (4 ed.). Philadelphia: Lippincott Williams \& Wilkins.

McKeary, M., \& Newbold, B. (2010). Barriers to care: The challenges for Canadian refugees and their health care providers. Journal of Refugee Studies, 23(4), 523-545. doi:10.1093/jrs/feq038

Meddings, F., \& Haith-Cooper, M. (2008). Culture and communication in ethically appropriate care. Nursing Ethics, 15(1), 52-61. doi:10.1177/0969733007083934

Mikkonen, J., \& Raphael, D. (2010). Social Determinants of Health: The Canadian Facts. Toronto: York University School of Health Policy and Management. Retrieved from, http://thecanadianfacts.org/the_canadian_facts.pdf

Moran, D. (2000). Introduction to Phenomenology. London: Routledge.

Morrison T.B., Wieland M.L., Cha S.S., Rahman A.S., Chaudhry R. (2012). Disparities in preventive health services among Somali immigrants and refugees. Journal of Immigrant Minority Health. 14 (6), 968-974. doi: 10.1007/s10903-012-9632-4

Nimmon, L. E. (2007). ESL-speaking immigrant women's disillusions: Voices of health care in Canada: An ethnodrama. Health Care for Women International, 28(4), 381-396. doi:10.1080/07399330601180016 
Northouse, L.L., \& Northouse, P.G. (1998). Health communication: Strategies for health professionals (3rd ed.). Stamford, CT: Appleton \& Lange.

Norlyk, A., Martinsen, B., \& Dahlberg, K. (2013). Getting to know patients' lived space. IndoPacific Journal of Phenomenology, 13(2), 1-12. doi:10.2989/IPJP.2013.13.2.5.1179A Nursing Council of New Zealand. (2011). Guidelines for cultural safety, the treaty of Waitangi and Maori health in nursing education and practice. Wellington, NZ: Author.

Patil, S., \& Davies, P. (2014). Use of google translate in medical communication: Evaluation of accuracy. BMJ: British Medical Journal, 349, g7392-g7392. doi:10.1136/bmj.g7392

Patton, M. Q. (2002). Qualitative research and evaluation methods. Thousand Oaks, California: Sage Publications.

Phillippi, J., \& Lauderdale, J. (2017). A guide to field notes for qualitative research: Context and conversation. Qualitative Health Research, 28(3), 381-388. doi:

$$
10.1177 / 1049732317697102
$$

Pottie, K., Ng, E., Spitzer, D., Mohammed, A., \& Glazier, R. (2008). Language proficiency, gender and self-reported health: An analysis of the first two waves of the longitudinal survey of immigrants to Canada. Canadian Journal of Public Health, 99(6), 505 - 510. Retrieved from http://ezproxy.lib.ryerson.ca/login?url=https://search-proquestcom.ezproxy.lib.ryerson.ca/docview/232001035?accountid=13631

Poureslami, I., Rootman, I., Doyle-Waters, M. M., Nimmon, L., \& FitzGerald, J. M. (2011). Health literacy, language, and ethnicity-related factors in newcomer asthma patients to Canada: A qualitative study. Journal of Immigrant and Minority Health, 13(2), 315-322. Retrieved from, https://doi-org.ezproxy.lib.ryerson.ca/10.1007/s10903-010-9405-x Probst, S., \& Imhof, L. (2016). Management of language discordance in clinical nursing practice - A critical review. Applied Nursing Research, 30(1), 158-163. 
doi: 10.1016/j.apnr.2015.07.005

Ramirez, D., Engel, K. G., \& Tang, T. S. (2008). Language interpreter utilization in the emergency department setting: A clinical review. Journal of Health Care for the Poor and Underserved, 19(2), 352-362. doi: 10.1353/hpu.0.0019

Reiners, G. M. (2012). Understanding the Differences between Husserl's (Descriptive) and Heidegger's (Interpretive) Phenomenological Research. J Nurs Care 1:119. doi:10.4172/2167-1168.1000119

Renfrew, M., Taing, E., Cohen, M. J., Betancourt, J. R., Pasinski, R., \& Green, A. R. (2013). Barriers to care for Cambodian patients with diabetes: Results from a qualitative study. Journal of Health Care for the Poor and Underserved, 24(2), 633-655. doi:10.1353/hpu.2013.0065

Rentmeester, C. (2018). Heidegger, communication, and healthcare. Medicine, Health Care and Philosophy, 21(3), 431-437. doi:10.1007/s11019-018-9823-4

Sears, J., Khan, K., Ardern, C. I., \& Tamim, H. (2013). Potential for patient-physician language discordance in Ontario. BMC Health Services Research, 13(1), 535-535. doi:10.1186/1472-6963-13-535

Segalowitz, N. \& Kehayia, E. (2011). Exploring the determinants of language barriers in health care (LBHC): Toward a research agenda for the language sciences. The Canadian Modern Language Review, 67 (4), 480-507. doi:10.3138/cmlr.67.4.480

Statistics Canada (2017a, August 2). Linguistic Diversity and multilingualism in Canadian homes. Retrieved from http://www12.statcan.gc.ca/census-recensement/2016/as-sa/98-200x/2016010/98-200-x2016010-eng.pdf 
Statistics Canada (2017b, August 17). An increasingly diverse linguistic landscape: Corrected data from the 2016 Census. Retrieved from http://www.statcan.gc.ca/dailyquotidien/170817/dq170817a-eng.pdf

Statistics Canada (2017c, October 25). Immigration and ethnocultural diversity: Key results from the 2016 Census. Retrieved from https://www150.statcan.gc.ca/n1/en/dailyquotidien/171025/dq171025b-eng.pdf?st=sW_K9Bb_

Streubert, H. J., \& Carpenter, D. R. (2011). Qualitative research in nursing: Advancing the humanistic imperative. (5th ed.). Philadelphia: Lippincott, Williams \& Wilkins.

Sultana, A., Aery, A., Kumar, N., Laher, N. (2018). Language interpretation services in health care settings in health care settings in the GTA. Retrieved from http://www.wellesleyinstitute.com/wp-content/uploads/2018/04/Language-InterpretationServices-in-the-GTA.pdf

Thomas, E., \& Magilvy, J. K. (2011). Qualitative rigor or research validity in qualitative research. Journal for Specialists in Pediatric Nursing, 16(2), 151-155. doi:10.1111/j.17446155.2011.00283.x

Topps, M., \& Strasser, R. F. (2010). When a community hospital becomes an academic health centre. Canadian Journal of Rural Medicine, 15(1), 19-25. Retrieved from http://ezproxy.lib.ryerson.ca/login?url=https://search-proquestcom.ezproxy.lib.ryerson.ca/docview/217581794?accountid=13631

Tuohy, D., Cooney, A., Dowling, M., Murphy, K., \& Sixsmith, J. (2013). An overview of interpretive phenomenology as a research methodology. Nurse Researcher, 20(6), 17-20. doi:10.7748/nr2013.07.20.6.17.e315 
van Manen, M. (1997). Researching lived experience: Human science for an action sensitive pedagogy. New York: Routledge.

van Manen, M. (2014). Phenomenology of practice: Meaning-giving methods in phenomenological research and writing. Walnut Creek: Left Coast Press.

van Manen, M., Higgins, I., \& Riet, P. (2016). A conversation with Max van Manen on phenomenology in its original sense. Nursing \& Health Sciences, 18(1), 4-7. doi:10.1111/nhs. 12274

Wilson, A. (2014). Being a practitioner: An application of heidegger's phenomenology. Nurse Researcher (2014+), 21(6), 28. doi:http://dx.doi.org/10.7748/nr.21.6.28.e1251

Whitman, M. V., \& Davis, J. A. (2009). Registered nurses' perceptions of cultural and linguistic hospital resources. Nursing Outlook, 57(1), 35-41. doi: 10.1016/j.outlook.2008.07.001

Williamson, M., \& Harrison, L. (2010). Providing culturally appropriate care: A literature review. International Journal of Nursing Studies, 47(6), 761-769. doi:

10.1016/j.ijnurstu.2009.12.012

World Health Organization. (n.d.). Health equity. Retrieved from http://www.who.int/topics/health_equity/en/

Wright, K.B., \& Moore, S.D. (2008). Applied health communication. Cresskill, NJ: Hampton Press. 